\author{
Universidade de São Paulo \\ Instituto de Física
}

\title{
Estudo do espalhamento e transferência elástica para o sistema ${ }^{7} \mathrm{Be}+{ }^{9} \mathrm{Be}$
}

\section{Uiran Umbelino da Silva}

\author{
Orientador: Prof ${ }^{\mathrm{a}}$. Dr ${ }^{\mathrm{a}}$. Kelly Cristina Cezaretto Pires
}

Dissertação de mestrado apresentada ao Instituto de Física para a obtenção do título de Mestre em Ciências

Banca Examinadora:

Prof $^{a}$. Dra . Kelly Cristina Cezaretto Pires (IFUSP)

Prof. Dr. Julian Marco Barbosa Shorto (IPEN/CNEN)

Prof. Dr. Andres Arazi (Tandar/Argentina)

São Paulo

2016 


\section{FICHA CATALOGRÁFICA \\ Preparada pelo Serviço de Biblioteca e Informação do Instituto de Física da Universidade de São Paulo}

Silva, Uiran Umbelino da

Estudo do espalhamento e transferência elástica para o sistema 7Be + 9Be. São Paulo, 2016.

Dissertação (Mestrado) - Universidade de São Paulo. Instituto de Física. Depto. de Física Nuclear

Orientador: Profa. Dra. Kelly Cristina Cezaretto Pires

Área de Concentração: Física

Unitermos: 1. Reações nucleares; 2. Espalhamento quasielástico; 3. Transferência elástica; 4. Fator espectroscópico; 5 . Nucleos radioativos.

USP/IF/SBI-095/2016 


\title{
University of São Paulo \\ Institute of Physics
}

\section{Elastic scattering and elastic transfer study of ${ }^{7} \mathrm{Be}+{ }^{9}$ Be system}

\section{Uiran Umbelino da Silva}

\author{
Advisor: Prof. Dr. Kelly Cristina Cezaretto Pires
}

Master's thesis submitted to the Institute of Physics to obtain the title of Master of Science

Examination Board:

Prof. Dr. Kelly Cristina Cezaretto Pires (IFUSP)

Prof. Dr. Julian Marco Barbosa Shorto (IPEN/CNEN)

Prof. Dr. Andres Arazi (Tandar/Argentina) 



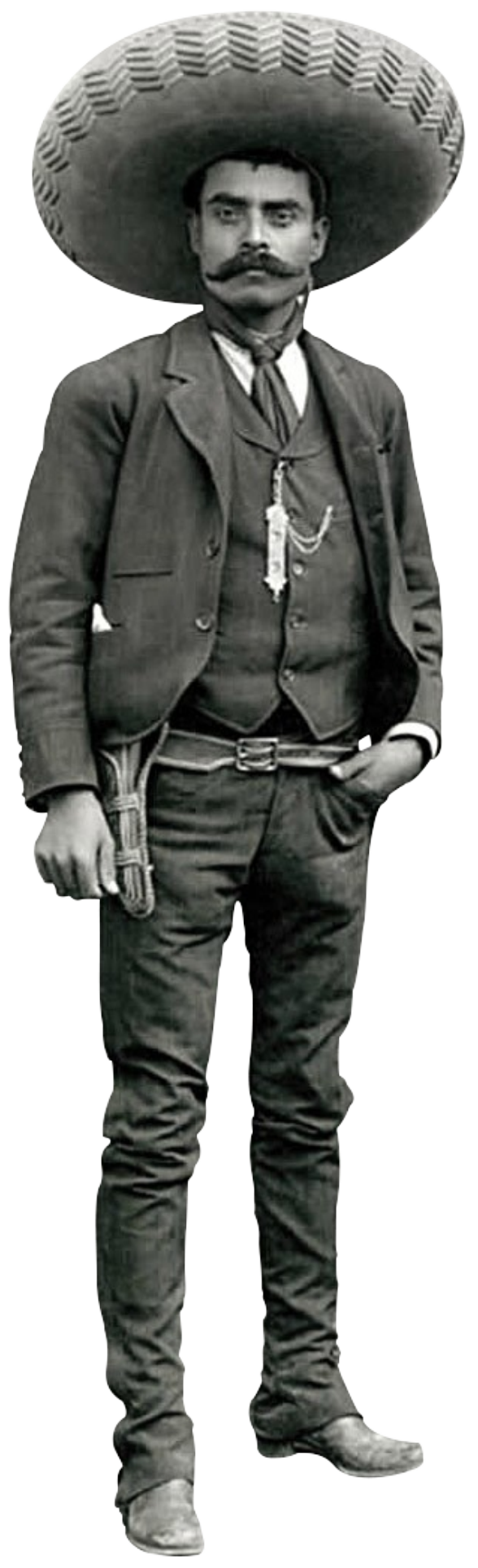

"Si no hay justicia para el pueblo, que no haya paz para el gobierno" Emiliano Zapata 
Esse trabalho é dedicado aos meus pais, irmão, amigos e todos aqueles que se desiludiram com o curso de Física. 


\section{Agradecimentos}

Primeiramente agradeço à minha família, meus pais Delmira Izabel de Jesus Silva e Cícero Umbelino da Silva e meu irmão Thiago da Silva, que me deram apoio incondicional em todos os momentos mais conturbados nesse difícil caminho até aqui. Sempre com muitas discussões, conversa, risadas e companheirismo.

À Profa. Dra. Kelly Cristina Cezaretto Pires, pela orientação, paciência e dedicação à este trabalho, que não seria possível sem ela.

Aos professores Prof. Dr. Rubens Lichtenthäler Filho, Profa. Dra. Alinka Lèpine-Szily, Prof. Dr. Marcos Aurelio Gonzales Alvarez e Prof. Dr. Valdir Guimarães pela ajuda em todos os momentos, desde a experiência até análise de dados.

Ao aluno de doutorado Valdir Bruneti Scarduelli pela ajuda e discussões.

Ao aluno de mestrado e companheiro de sala George Abud Scotton, que acompanhou toda a trajetória até esse momento.

Aos membros do Grupo de Reações Diretas e Núcleos Exóticos: Profa. Dra. Marlete Assunção, Profa. Dra. Viviane Morcelle, Prof. Dr. Julian M. B. Shorto, Dr. Rubén Pampa Condori, Dr. Appannababu Selaboina e Dr. Yanyun Yang que ajudaram direta e indiretamente na realização deste trabalho.

À todos os funcionários do Edifício Oscar Sala que, com seu esforço, permitiram a realização deste trabalho.

Aos meus amigos de infância Gabriel Neves Martins, Gregório Habyak Prado e Letírcio José de Almeida Filho por estarem sempre presentes, seja para discussões políticas ou pra uma conversa descontraída no bar. E que a luta continue.

Aos meus amigos da "Panela": que sempre me trouxeram bons momentos com muita alegria e "Maralimão".

Aos meus amigos do IFUSP, IPEN e IAG que, desde 2008, partilham dessa 
difícil trajetória. Àqueles que ainda estudam física, mas principalmente àqueles que desistiram pelos mais diversos motivos, eu carrego seus sonhos comigo.

Aos meus companheiros de esporte do HandBöhr: Thiago de Castro, Breno Buckup, Marcel de Paula, Renato Scher, Arthur Pelegrini, Vitor Baricelli, Rafael Zimak, Arlino Junior, Filipe Terra Nova, Juliana Cocentino, Hyana Labanca, Beatriz Baeta, Felipe Miyazato, Bruna Alves, Wagner Fazio, Florence de Almeida, Gabriela Wasconcellos, Rafael Rezende, Hudson Lodi, Hudson Novak (Djoko), Janine Araujo, João Jota, Pâmela Souto, Pedro Panda, Renan Carrilo, Ruth Staschower, Frederico Almeida (Shrek) e Thais Andrade, que estiveram presentes nos momentos mais difíceis e em alguns dos momentos mais alegres, fazendo com que esses anos de IFUSP fossem mais leves e divertidos.

À Bárbara dos Santos Pereira Meyer pela ajuda na escrita dessa dissertação, pelo companheirismo e paciência nesse último, e conturbado, ano de Mestrado.

À CNPq pelo suporte financeiro. 


\section{Resumo}

Foram realizadas medidas de espalhamento para o sistema ${ }^{7} \mathrm{Be}+{ }^{9} \mathrm{Be}$ em $\mathrm{E}_{\mathrm{CM}}=13$ $\mathrm{MeV}\left(\mathrm{E}_{\mathrm{lab}}=23,1 \mathrm{MeV}\right)$. A experiência foi realizada utilizando o acelerador Pelletron do Instituto de Física da Universidade de São Paulo em conjunto com o sistema RIBRAS (Radioactive Ion Beams in Brasil). As seções de choque ${ }^{9} \mathrm{Be}\left({ }^{7} \mathrm{Be},{ }^{7} \mathrm{Be}\right){ }^{9} \mathrm{Be}$ e de transferência elástica ${ }^{9} \mathrm{Be}\left({ }^{7} \mathrm{Be},{ }^{9} \mathrm{Be}\right){ }^{7} \mathrm{Be}$ foram obtidas e utilizadas na construção da distribuição angular completa, na região angular entre $15^{\circ}$ até $160^{\circ}$. Cálculos com modelo óptico foram realizados utilizando diversos potenciais encontrados na literatura. Devido à energia do primeiro estado excitado do ${ }^{7} \mathrm{Be}\left(\mathrm{E}_{x}=0,429 \mathrm{MeV}\right)$ ser menor que a resolução energética do experimento, a seção de choque elástica não pode ser separada experimentalmente da inelástica, porém, através de cálculos de canais acoplados foi comprovado que o espalhamento inelástico não tem influência considerável na distribuição angular elástica. Por fim, através de cálculos de DWBA para a transferência elástica, foram obtidos fatores espectroscópicos de dois nêutrons no ${ }^{9} \mathrm{Be}$. Os resultados indicam que os potenciais de espalhamento e do estado ligado têm grande influência no canal de transferência elástica.

Palavras-chave: espalhamento quasielástico, transferência elástica, núcleos leves, núcleos radioativos, feixes radioativos, sistema RIBRAS, Acelerador Pelletron. 


\section{Abstract}

${ }^{7} \mathrm{Be}+{ }^{9} \mathrm{Be}$ scattering measurements were performed at $\mathrm{E}_{\mathrm{CM}}=13 \mathrm{MeV}\left(\mathrm{E}_{\mathrm{lab}}=\right.$ 23.1 MeV). The experiment was carried out in the 8UD Pelletron accelerator of the Institute of Physics of the University of São Paulo with the RIBRAS (Radioactive Ion Beams in Brasil) system. The elastic ${ }^{9} \mathrm{Be}\left({ }^{7} \mathrm{Be},{ }^{7} \mathrm{Be}\right){ }^{9} \mathrm{Be}$ and elastic transfer ${ }^{9} \mathrm{Be}\left({ }^{7} \mathrm{Be},{ }^{9} \mathrm{Be}\right){ }^{7} \mathrm{Be}$ cross sections were obtained and used to construct the complete angular distribution, in the angular range from $15^{\circ}$ to $160^{\circ}$. Optical model calculations were performed using several optical potentials from the literature. Due to the first low lying excited state of ${ }^{7} \mathrm{Be}\left(\mathrm{E}_{x}=0.429 \mathrm{MeV}\right)$ the elastic cross section cannot be experimentally separated of the inelastic part. However, coupled channel calculations show that the contribution of the inelastic scattering has no major influence on the elastic angular distribution. Finally, the DWBA calculations for elastic transfer provide the two neutrons spectroscopic factors in ${ }^{9} \mathrm{Be}$. The results indicate that the transfer channel is sensitive to the bound state and optical potentials used in the analysis.

Keywords: elastic scattering, elastic transfer, light nuclei, radioactive nuclei, RIBRAS system, Pelletron. 


\section{Lista de Figuras}

1.1 Esquema de um espalhamento elástico, onde o núcleo incidente é o mesmo do núcleo espalhado. . . . . . . . . . . . . . . . . . . 2

1.2 Carta de nuclídeos, formada em 1996, mostrando os núcleos estáveis, instáveis e os previstos mas ainda não descobertos [12] . . . . . . . . 3

2.1 Esquema de funcionamento da fonte MC-SNICS [18]. . . . . . . . . . 8

2.2 Esquema do acelerador Pelletron localizado no edifício Oscar Sala [20]. 9

2.3 Esquema da geração de alta tensão no acelerador Pelletron (adaptado de $[21]) \ldots \ldots \ldots \ldots \ldots$

2.4 Esquema do acelerador eletrostático do laboratório Pelletron. Imagem de J.C. Terassi. . . . . . . . . . . . . . . . . . . . 12

2.5 Esquema do sistema RIBRAS [5] . . . . . . . . . . . . . . 13

2.6 Esquema mostrando o alvo primário e o copo de Faraday [5] . . . . . . 14

2.7 Exemplo de esquema dos colimadores e bloqueadores posicionados próximos do primeiro solenoide. . . . . . . . . . . . . 16

2.8 Foto da câmara central, mostrando (a) dois telescópios montados e (b) a haste para movimentação do prato giratório. . . . . . . . . . . 17

2.9 (a) Esquema da montagem dos detectores no prato giratório e (b) exemplo de um telescópio indicando o (1) colimador, (2) suporte, (3) detector $\Delta \mathrm{E}$, (4) detector $\mathrm{E}$, (5) tampa de fixação [2] . . . . . . . . . 19

2.10 Exemplos de espectros (a) mono e (b) biparamétricos obtidos no experimento para o espalhamento quasielástico ${ }^{7} \mathrm{Be}+{ }^{9} \mathrm{Be}$. . . . . . . . 20

2.11 Esquema da eletrônica utilizada no experimento realizado neste trabalho. 
3.1 Trajetórias calculadas com o algoritmo TWSP [11] . . . . . . . . 25

3.2 Gráfico da taxa de produção x corrente do solenoide. . . . . . . . . . 26

3.3 Espectros biparamétricos obtidos com feixe de ${ }^{7} \mathrm{Be}$ e alvo de (a) ${ }^{197} \mathrm{Au}$ e (b) ${ }^{9}$ Be para $\theta_{\text {lab }}=15^{\circ}$. dE é a perda de energia no detector $\Delta \mathrm{E}$ e E é a energia residual. . . . . . . . . . . . . . . . . . 27

3.4 Retas calibração para os detectores (a) $\mathrm{E}_{1}$ (b) $\mathrm{E}_{2}$ (c) $\Delta \mathrm{E}_{2}$ (d) $\mathrm{E}_{3}$ (e) $\Delta \mathrm{E}_{3}$ (f) $\mathrm{E}_{4}$. Os pontos identificados como alfas representam os dados obtidos pela calibração com a fonte de ${ }^{241} \mathrm{Am}$, enquanto os identificados como $4 \mathrm{He} 2+$ representam as partículas alfas contaminantes obtidas no experimento. Para os detectores $\mathrm{E}_{2}$ e $\mathrm{E}_{3}$, os pontos mostram a calibração realizada com e sem o detector $\Delta \mathrm{E}$ na frente. . 28

3.5 Espectros biparamétricos $\Delta \mathrm{E} \times \mathrm{E}_{\text {total }}$ obtidos com feixe de ${ }^{7}$ Be e alvo de (a) ${ }^{197} \mathrm{Au}$ e (b) ${ }^{9} \mathrm{Be}$ para $\theta_{\text {lab }}=15^{\mathrm{o}} \ldots \ldots \ldots . . \ldots 29$

3.6 Variação da eficiência de produção ao longo do experimento. Mostrando a média $\left(\bar{\epsilon} \approx 6 \times 10^{-9}\right)$ obtida ao longo do experimento. . . . . 31

3.7 Distribuição angular ${ }^{7} \mathrm{Be}+{ }^{197} \mathrm{Au}$ para $\mathrm{E}_{\mathrm{lab}}=23,3 \mathrm{MeV}$, com e sem a correção angular utilizando o código RIBRAS I. . . . . . . . . . . . . 33

3.8 Distribuição angular normalizada (a) e absoluta (b) para o espalhamento quasielástico ${ }^{7} \mathrm{Be}+{ }^{9} \mathrm{Be}$ a $\mathrm{E}_{\mathrm{lab}}=23,1 \mathrm{MeV}$. . . . . . . . . . . . 34

3.9 Comparação com dados obtidos por (a) Mukha et al [2] e (b) S. Verma et al [1], com as respectivas energias.

3.10 Espectro biparamétrico $\Delta \mathrm{E} \times \mathrm{E}_{\text {Total }}$ obtido com feixe de ${ }^{7} \mathrm{Be}$ e alvo de ${ }^{9} \mathrm{Be}$ para $\theta_{\mathrm{CM}}=15^{\circ}$. É possível observar as contagens referentes ao processo de transferência elástica ${ }^{9} \mathrm{Be}\left({ }^{7} \mathrm{Be},{ }^{9} \mathrm{Be}\right){ }^{7} \mathrm{Be}$. . . . . . . . 36

3.11 Distribuição angular da reação de transferência ${ }^{9} \mathrm{Be}\left({ }^{7} \mathrm{Be},{ }^{9} \mathrm{Be}\right){ }^{7} \mathrm{Be}$ somada à detecção de ${ }^{9}$ Be de recuo em ângulos dianteiros. . . . . . . . . 37

3.12 Distribuição angular do espalhamento quasielástico ${ }^{7} \mathrm{Be}+{ }^{9} \mathrm{Be}$ somada à contribuição referente à transferência elástica em ângulos traseiros.

4.1 Espalhamento através de reações diretas e pela formação de núcleo composto. . . . . . . . . . . . . . . . . 40 
4.2 Forma do potencial de Woods-Saxon. . . . . . . . . . . . . . . 46

4.3 Geometria utilizada no potencial de São Paulo. . . . . . . . . . . . . 47

4.4 Esquema generalizado do modelo coletivo. . . . . . . . . . . . . . . . 51

4.5 Esquema generalizado do modelo de cluster. . . . . . . . . . . . . . 52

4.6 Relação cinemática do (a) espalhamento elástico e (b) transferência elástica, ambos no referencial do centro de massa. . . . . . . . . . . 55

5.1 Distribuição angular do espalhamento quasielástico ${ }^{7} \mathrm{Be}+{ }^{9} \mathrm{Be}$ para $\mathrm{E}_{\mathrm{lab}}=23,1 \mathrm{MeV}$ comparada com cálculos de modelo óptico utilizando fator de forma Woods-Saxon. As identificações de (a) a (j) se referem aos potenciais apresentados na Tabela 5.1. . . . . . . . . . . . 59

5.2 Distribuição angular do espalhamento quasielástico ${ }^{7} \mathrm{Be}+{ }^{9} \mathrm{Be}$ para $\mathrm{E}_{\text {lab }}=23,1 \mathrm{MeV}$ comparada com cálculos de modelo óptico utilizando fator de forma Woods-Saxon, variando as profundidades real e imaginária. As identificações de (a) a (j) se referem aos potenciais apresentados na Tabela 5.2. . . . . . . . . . . . . . . . . . . 61

5.3 Distribuição angular do espalhamento quasielástico ${ }^{7} \mathrm{Be}+{ }^{9} \mathrm{Be}$ para $\mathrm{E}_{\text {lab }}=23,1 \mathrm{MeV}$ comparada com os cálculos de modelo óptico utilizando fator de forma Woods-Saxon, variando todos os parâmetros. As identificações (k) e (l) se referem aos potenciais apresentados na Tabela 5.3. . . . . . . . . . . . . . . . . 63

5.4 Potenciais obtidos com o código SPOMC. . . . . . . . . . . . . . 64

5.5 Distribuição angular do espalhamento quasielástico ${ }^{7} \mathrm{Be}+{ }^{9} \mathrm{Be}$ para $\mathrm{E}_{\mathrm{lab}}=23,1 \mathrm{MeV}$ comparada com os cálculos realizados com o modelo óptico utilizando o potencial de São Paulo. . . . . . . . . . . . . . . . 66

5.6 Distribuição angular do espalhamento quasielástico ${ }^{7} \mathrm{Be}+{ }^{9} \mathrm{Be}$ para $\mathrm{E}_{\mathrm{lab}}=23,1 \mathrm{MeV}$ comparada com os cálculos de modelo óptico utilizando o potencial de São Paulo na parte real e Woods-Saxon na parte imaginária. 
5.7 Distribuição angular do espalhamento quasielástico ${ }^{7} \mathrm{Be}+{ }^{9} \mathrm{Be}$ para $\mathrm{E}_{\text {lab }}=23,1 \mathrm{MeV}$ comparada com os cálculos realizados com o formalismo de canais acoplados. . . . . . . . . . . . . . . . 69

5.8 Distribuição angular do espalhamento quasielástico ${ }^{7} \mathrm{Be}+{ }^{9} \mathrm{Be}$ para $\mathrm{E}_{\mathrm{lab}}=23,1 \mathrm{MeV}$ comparada com os cálculos realizados com o formalismo de canais acoplados variando as profundidades real e imaginária dos potenciais. . . . . . . . . . . . . . . . 71

5.9 Distribuição angular do espalhamento quasielástico ${ }^{7} \mathrm{Be}+{ }^{9} \mathrm{Be}$ para $\mathrm{E}_{\mathrm{lab}}=23,1 \mathrm{MeV}$ comparada com os cálculos de DWBA variando a amplitude espectroscópica $A$. Para a parte elástica foi utilizado um potencial do tipo Woods-Saxon (l) obtido nesse trabalho. . . . . . . . 73

5.10 Distribuição angular do espalhamento quasielástico ${ }^{7} \mathrm{Be}+{ }^{9} \mathrm{Be}$ para $\mathrm{E}_{\text {lab }}=23,1 \mathrm{MeV}$ comparada com cálculos de DWBA variando a amplitude espectroscópica $A$. Foi obtido o resultado $A=0,36(5)$ e

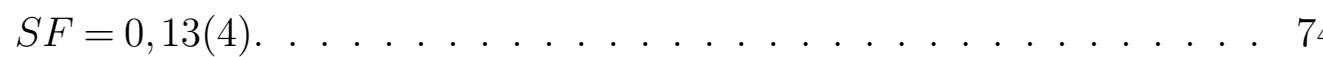

5.11 Distribuição angular do espalhamento quasielástico ${ }^{7} \mathrm{Be}+{ }^{9} \mathrm{Be}$ para $\mathrm{E}_{\mathrm{lab}}=23,1 \mathrm{MeV}$ comparada com os cálculos de DWBA variando a amplitude espectroscópica $A$. Para a parte elástica foi utilizado um potencial do tipo São Paulo para a parte real e Woods-Saxon para a parte imaginária. . . . . . . . . . . . . . . . . . 75

5.12 Distribuição angular do espalhamento quasielástico ${ }^{7} \mathrm{Be}+{ }^{9} \mathrm{Be}$ para $\mathrm{E}_{\mathrm{lab}}=23,1 \mathrm{MeV}$ comparada com os cálculos de DWBA variando a amplitude espectroscópica $A$. Foi obtido o resultado $A=0,7(1)$ e

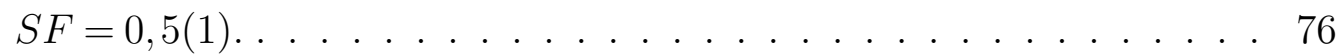

5.13 Distribuição angular do espalhamento quasielástico ${ }^{7} \mathrm{Be}+{ }^{9} \mathrm{Be}$ para $\mathrm{E}_{\mathrm{lab}}=23,1 \mathrm{MeV}$ comparada com os cálculos de DWBA com as duas amplitudes espectroscópica obtidas neste trabalho $A_{1}=0,36(5)$ e

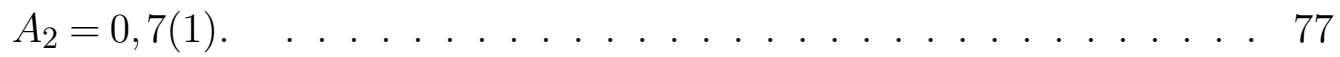




\section{Lista de Tabelas}

1.1 Reações importantes relacionadas ao núcleo ${ }^{7} \mathrm{Be}$, mostrando a temperatura de dominância e a energia dos neutrinos liberados [15]. . . . 4

1.2 Algumas características do núcleo ${ }^{7} \mathrm{Be} \ldots \ldots \ldots \ldots$

2.1 Algumas reações utilizadas para produção de feixes no sistema RIBRAS. 15

2.2 Especificações dos detectores utilizados. . . . . . . . . . . . . . 18

2.3 Distâncias do alvo-detetor e ângulos sólidos obtidos ${ }^{1}$. . . . . . . . . . 19

3.1 Espessuras dos alvos utilizados neste trabalho. . . . . . . . . . . . . . 24

3.2 Resultado do ajuste das retas de calibração. As incertezas foram obtidas pelo programa GNUPLOT [28], usado para construir os gráficos deste trabalho. . . . . . . . . . . . . . . . . . . . . . . . . 29

5.1 Parâmetros dos potenciais ópticos, com fator de forma Woods-Saxon, utilizados nos cálculos. Tipo identifica os potenciais volumétricos (Vol.) e superficiais (Sup.). Os valores dos raios reduzidos foram convertidos em raio absoluto usando as relações $R=r_{0}\left(A_{T}^{1 / 3}+A_{P}^{1 / 3}\right)^{1}$ e $N$ é o número de dados. . . . . . . . . . . . . . . 58

5.2 Parâmetros do potencial óptico, com fator de forma Woods-Saxon, obtidos com a variação das profundidades $V$ e $W$, minimizando o valor de $\chi^{2} / N$. Os valores dos raios reduzidos foram convertidos em raio absoluto usando a relação $R=r_{0}\left(A_{T}^{1 / 3}+A_{P}^{1 / 3}\right) \ldots$. . . . . . . . 60 
5.3 Parâmetros do potencial óptico, com fator de forma Woods-Saxon, obtidos pela variação de todos os parâmetros, minimizando o valor de $\chi^{2} / N$. Os valores dos raios reduzidos foram convertidos em raio absoluto usando a relação $R=r_{0}\left(A_{T}^{1 / 3}+A_{P}^{1 / 3}\right) \ldots \ldots$. . . . . . 62

5.4 Parâmetros do potencial óptico, usando como fator de forma o potencial de São Paulo, obtidos pela variação das normalizações $N_{\mathbb{R}}$ e $\mathrm{N}_{\mathbb{I}}$, minimizando o valor local do $\chi^{2}$. . . . . . . . . . . . 65

5.5 Parâmetros do potencial óptico, usando o potencial de São Paulo na parte real e Woods-Saxon na parte imaginária. . . . . . . . . . . . 67

5.6 Parâmetros dos potenciais utilizados para o cálculo do espalhamento quasielástico. Para o potencial ${ }^{4} \mathrm{He}+{ }^{3} \mathrm{He}$, assim como a parte imaginária do potencial $\left({ }^{4} \mathrm{He}+{ }^{9} \mathrm{Be}\right)_{b}$, foi usada um potencial na forma Gaussiana: $V(r)=-V_{0} e^{-\left(r / r_{0}\right)^{2}} \ldots \ldots \ldots \ldots$

5.7 Parâmetros dos potenciais obtidos através de uma busca automática no cálculo do espalhamento quasielástico através do formalismo de canais acoplados. . . . . . . . . . . . . . . . 70

5.8 Potenciais usados para os cálculos de DWBA. . . . . . . . . . . . . . 72

5.9 Amplitudes espectroscópicas obtidas com o potencial Woods-Saxon

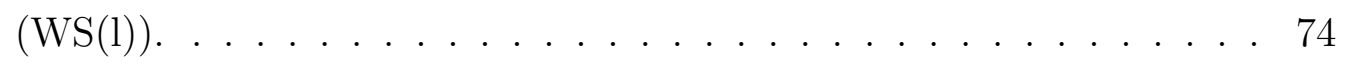

5.10 Amplitudes espectroscópicas obtidas com o potencial São Paulo na parte real e Woods-Saxon na parte imaginária. . . . . . . . . . . . 75

5.11 Seções de choque de reação dos potenciais que melhor reproduzem os dados experimentais do sistema ${ }^{7} \mathrm{Be}+{ }^{9} \mathrm{Be}$ para $\mathrm{E}_{\mathrm{lab}}=23,1 \mathrm{MeV}$. . . 78

5.12 Valores médios para as seções de choque de reações para potenciais encontrados na literatura. 


\section{Sumário}

Resumo iii

$\begin{array}{lll}\text { Abstract } & \text { v }\end{array}$

Lista de Figuras $\quad$ x

Lista de Tabelas $\quad$ xii

1 Introdução 1

2 Descrição Experimental $\quad 7$

2.1 Fonte SNICS . . . . . . . . . . . . . . . . . . . . 7

2.2 Acelerador Pelletron . . . . . . . . . . . . . . . . . . . . 9

2.3 Sistema RIBRAS . . . . . . . . . . . . . . . . . . 13

2.3.1 Câmara de Espalhamento e Sistema de Detecção . . . . . . . . 17

2.3.2 Sistema de Aquisição . . . . . . . . . . . . . . . . . . . . 20

3 O Experimento ${ }^{7} \mathrm{Be}+{ }^{9} \mathrm{Be} \quad 23$

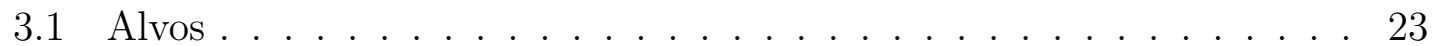

3.2 Taxa de Produção . . . . . . . . . . . . . . . . . . . 25

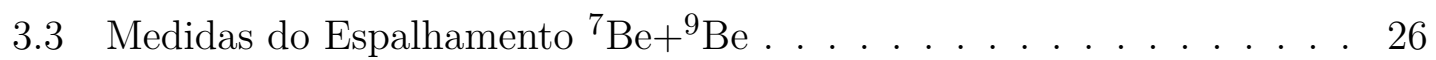

3.3.1 Calibração dos Detectores . . . . . . . . . . . . . . . 27

3.3 .2 Eficiência de Produção . . . . . . . . . . . . . . . . 30

3.4 Distribuições Angulares . . . . . . . . . . . . . . . . . . . . . . . 32

3.4 .1 Correção Angular . . . . . . . . . . . . . . . . . . . . . . . . 32

3.4 .2 Distribuições Angulares Elásticas . . . . . . . . . . . . . 32 
3.4.2.1 Comparação com dados da literatura . . . . . . . . 35

3.4 .3 Transferência Elástica . . . . . . . . . . . . . . . 36

4 Fundamentos Teóricos $\quad 39$

4.1 Reações Diretas . . . . . . . . . . . . . . . . . . . . 39

4.2 Espalhamento Elástico . . . . . . . . . . . . . . . . . . 40

4.2.1 Espalhamento Rutherford . . . . . . . . . . . . 42

4.2.2 Espalhamento por um potencial adicional de curto alcance . . 43

4.2 .3 Modelo Óptico $(\mathrm{OM}) \ldots \ldots$. . . . . . . . . . . . 44

4.2.3.1 Potenciais Ópticos . . . . . . . . . . . . 45

4.2.3.2 Potencial Woods-Saxon . . . . . . . . . . . . 46

4.2.3.3 Potencial de São Paulo . . . . . . . . . . . . . . . 47

4.3 Espalhamento Inelástico _ . . . . . . . . . . . . . . . . . . 48

4.3.1 Método de Canais Acoplados (CC) . . . . . . . . . . . . 48

4.3.1.1 Modelo de Camadas . . . . . . . . . . . . 50

4.3.1.2 Modelo Coletivo . . . . . . . . . . . . 50

4.3.1.3 Modelo de Partícula Única (Cluster) . . . . . . . . . 51

4.4 Aproximação de Born de Ondas Distorcidas (DWBA) . . . . . . . . . 52

4.4 .1 Transferência Elástica . . . . . . . . . . . . . . . 54

5 Análise da Distribuição ${ }^{7} \mathrm{Be}+{ }^{9} \mathrm{Be} \quad 57$

5.1 Cálculos com Modelo Óptico . . . . . . . . . . . . . . . . 57

5.1 .1 Potencial de Woods-Saxon . . . . . . . . . . . . . 57

5.1 .2 Potencial de São Paulo . . . . . . . . . . . . . . . . . . . . 64

5.1 .3 Potencial Misto . . . . . . . . . . . . . . 67

5.2 Cálculos com Canais Acoplados (CC) . . . . . . . . . . . . . . . 68

5.3 Cálculos de DWBA . . . . . . . . . . . . . . . . . . . . . . 72

5.4 Seção de Choque de Reação . . . . . . . . . . . . . . . . . . . . . 78

6 Conclusões $\quad 81$

Referências Bibliográficas $\quad 83$ 
$\begin{array}{lr}\text { A Inputs FRESCO } & 89\end{array}$

A.1 Modelo Óptico - Woods-Saxon . . . . . . . . . . . . . . . . . . . . . 90

A.2 Modelo Óptico - São Paulo . . . . . . . . . . . . . . . . . . . . 91

A.3 Modelo Óptico - Misto . . . . . . . . . . . . . . . . . . . . . 92

A.4 Canais Acoplados . . . . . . . . . . . . . . . . 93

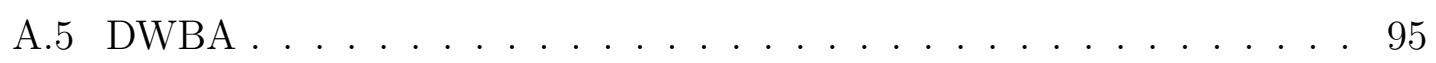




\section{Capítulo 1}

\section{Introdução}

Os primeiros registros a respeito da composição da matéria remontam-se à era Clássica, onde o trabalho de diversos filósofos culminou no pensamento de Demócrito, um dos primeiros a utilizar o termo átomo (do grego indivisível). Em 1803, John Dalton formulou que elementos químicos diferentes seriam formados por átomos diferentes. Em 1896, Henri Becquerel descobriu a radioatividade trabalhando em materiais fosforescentes. Em 1897, Joseph John Thomson descobriu o elétron e, com isso, formulou o que seria conhecido posteriormente como Modelo do Pudim de Passas. Já em 1909, Geiger e Marsden estudaram o espalhamento de partículas alfa em folhas de Ouro e formularam que os átomos são muito pequenos se comparados às distâncias interatômicas. Em 1911, Ernest Rutherford demonstrou experimentalmente que elétrons (cargas negativas) orbitam uma massa concentrada, o núcleo (carga positiva). Finalmente, em 1913, Niels Bohr usou a interação elétrica de Coulomb para formular o Modelo de Bohr e explicar as linhas de emissão do Hidrogênio.

Após o Modelo de Bohr muito esforço foi direcionado para o entendimento do núcleo dos átomos, resultando na primeira transmutação de matéria usando elementos naturalmente radioativos (1919 por Rutherford) e partículas artificialmente aceleradas (1932 por Cockcroft e Walton), também na descoberta dos prótons (1919 por Rutherford) e nêutrons (1932 por James Chadwick) e a formulação (1934 por Fermi) e observação (1939 por Hahn e Strassmann) do evento conhecido como fissão nuclear. Esses esforços, teóricos e experimentais, abriram portas para diversos estudos visando desvendar as estruturas nucleares. Ainda durante o século XX, muitos 
modelos nucleares foram propostos, dentre eles o Modelo Coletivo (Gota Líquida) e o Modelo de Camadas. Porém, nenhum dos modelos explica com precisão todas as características do núcleo atômico, tornando importante o estudo das reações nucleares.

Dentre os diversos tipos de colisões, o espalhamento elástico é muito estudado [1, $2,3,4,5]$, sendo o processo mais simples, onde o núcleo incidente e o núcleo espalhado são os mesmos. Esse tipo de espalhamento é importante, pois permite um estudo direto do núcleo de interesse, visto que não há uma forte interação entre o núcleo incidente e os núcleons mais internos do núcleo alvo. Assim, pode-se fazer uma caracterização da superfície do núcleo de interesse. Os dados experimentais (seção de choque) devem ser reproduzidos pelos potenciais nucleares teóricos, fornecendo importantes informações sobre a geometria nuclear. A Fig. 1.1 mostra um esquema de um espalhamento elástico.

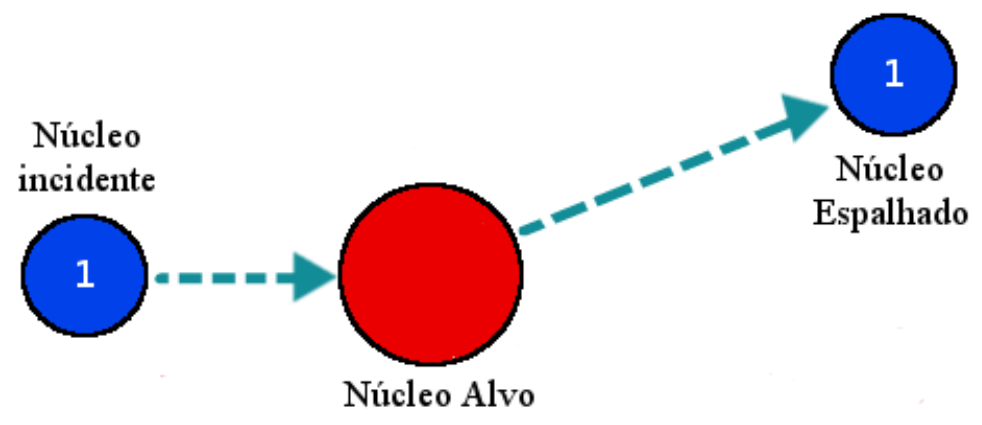

Figura 1.1: Esquema de um espalhamento elástico, onde o núcleo incidente é o mesmo do núcleo espalhado.

Outro tipo bastante estudada é a transferência $[6,7,8,9]$, onde há uma troca de núcleons entre o projétil e o alvo. A reação de pick-up ocorre quando há transferência de núcleons do alvo para o projétil e a reação de stripping ocorre na quando a transferência acontece do projétil para o alvo. Com esse tipo de colisão é possível obter informações sobre a normalização da função de onda dos núcleos reagentes. Um caso importante é a transferência elástica, onde as partículas espalhadas são idênticas às incidentes.

Os elementos são uma combinação específica de nêutrons e prótons unidos pela 
ação da força nuclear forte. Essa combinação fornece o tipo de elemento e, somada à interação Coulombiana dos prótons, se serão ou não estáveis ${ }^{1}$. Atualmente, cerca de 300 núcleos estáveis e mais de 3000 núcleos instáveis são conhecidos, porém milhares ainda desconhecidos são previstos por cálculos de massa e energia de ligação nuclear.

O estudo das estruturas e reações nucleares, envolvendo núcleos estáveis e instáveis $[10,11]$, tem grande importância para o entendimento da composição e abundância dos elementos no universo. A Fig. 1.2 mostra a carta de nuclídeos em função do número de prótons e nêutrons.

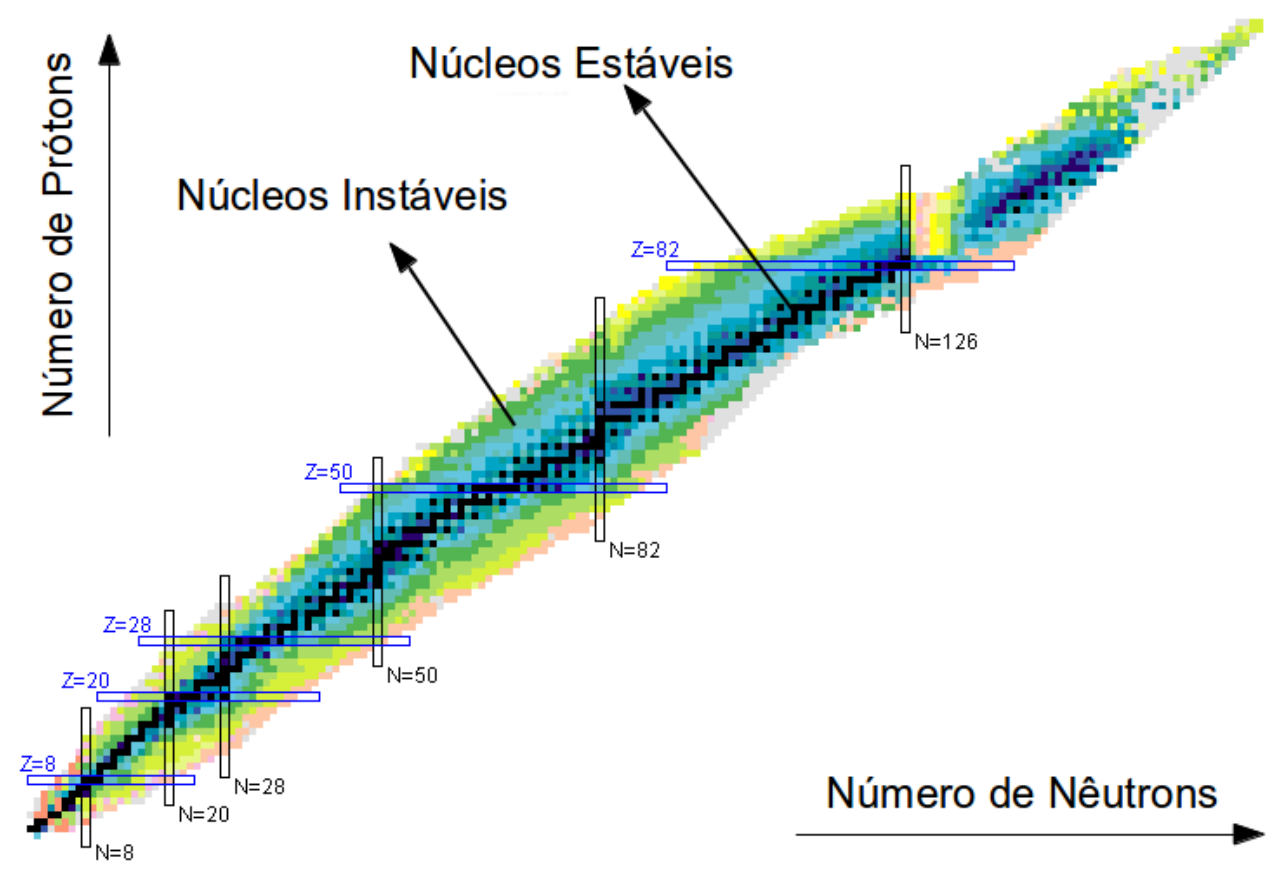

Figura 1.2: Carta de nuclídeos, formada em 1996, mostrando os núcleos estáveis, instáveis e os previstos mas ainda não descobertos [12].

Recentemente, a possibilidade de produção de feixes radioativos trouxe grandes avanços para o entendimento da estrutura nuclear e mecanismos de reação envolvendo esse tipo de elementos. Com isso, o interesse em núcleos longe da linha de estabilidade tem aumentado. O sistema RIBRAS [13, 14] é usado para a produção e focalização de feixes radioativos. Esses núcleos, ricos em prótons ou nêutrons, têm

\footnotetext{
${ }^{1}$ Para elementos leves, essa estabilidade se dá quando o número de prótons $(Z)$ está próximo do número de nêutrons $(A-Z)$. Conforme a massa elementar aumenta, a estabilidade ocorre para isótopos com número de nêutrons maior do que o número de prótons.
} 
características incomuns, como densidades nucleares anômalas ou halo nuclear ${ }^{2}$.

Nesse trabalho foi estudado o espalhamento ${ }^{7} \mathrm{Be}+{ }^{9} \mathrm{Be}$ em $\mathrm{E}_{\mathrm{lab}}=23,1 \mathrm{MeV}\left(\mathrm{E}_{\mathrm{CM}}\right.$ $=13,0 \mathrm{MeV}$ ). A energia da barreira Coulombiana para esse sistema no referencial do centro de massa é de 4,44 MeV. O ${ }^{7}$ Be é um importante núcleo no modelo de produção energética solar, pois é um isótopo relativamente leve, fazendo parte do chamado grupo do Lítio $(\mathrm{A}=7-12)$, que compreende cerca de $10^{-6 \%}$ do universo conhecido, sendo o grupo mais abundante após o Hidrogênio (74\%) e o Hélio (25\%) [15]. Esse núcleo está diretamente relacionado à produção de aproximadamente $9 \%$ da luminosidade solar através do ciclo próton-próton II (pp II), e de aproximadamente $47 \%$ dos neutrinos de alta energia pelo ciclo próton-próton III (pp III). A Tabela 1.1 mostra as reações envolvidas e as respectivas informações.

Tabela 1.1: Reações importantes relacionadas ao núcleo ${ }^{7} \mathrm{Be}$, mostrando a temperatura de dominância e a energia dos neutrinos liberados [15].

Ciclo \begin{tabular}{ccc} 
Reações envolvidas & Temperatura $(\mathrm{MK})$ & $\mathrm{E}_{\nu}(\mathrm{MeV})$ \\
pp II $\left\{\begin{array}{c}{ }^{3} \mathrm{He}+{ }^{4} \mathrm{He} \rightarrow{ }^{7} \mathrm{Be}+\gamma \\
{ }^{7} \mathrm{Be}+e^{-} \rightarrow{ }^{7} \mathrm{Li}+\nu_{e} \\
{ }^{7} \mathrm{Li}+{ }^{1} \mathrm{H} \rightarrow{ }^{4} \mathrm{He}+{ }^{4} \mathrm{He}\end{array}\right.$ & $\begin{array}{l}90 \%-0,861 \\
10 \%-0,383\end{array}$ \\
pp III $\left\{\begin{array}{l}{ }^{3} \mathrm{He}+{ }^{4} \mathrm{He} \rightarrow{ }^{7} \mathrm{Be}+\gamma \\
{ }^{7} \mathrm{Be}+{ }^{1} \mathrm{H} \rightarrow{ }^{8} \mathrm{~B}+\gamma \\
{ }^{8} \mathrm{~B} \rightarrow{ }^{8} \mathrm{Be}+e^{+}+\nu_{e} \\
{ }^{8} \mathrm{Be} \rightarrow{ }^{4} \mathrm{He}+{ }^{4} \mathrm{He}\end{array}\right.$ & $>23$ \\
\hline
\end{tabular}

O núcleo ${ }^{7}$ Be também é o núcleo espelho do ${ }^{7} \mathrm{Li}$, com estado ligado $(1 / 2)^{-} \mathrm{e}$ energia de excitação de aproximadamente $0,43 \mathrm{MeV}$ [16], o que permite estudos de simetria entre os dois, já que, no mesmo estado, o ${ }^{7} \mathrm{Li}$ tem energia de excitação de aproximadamente $0,48 \mathrm{MeV}$. A energia de break-up do decaimento ${ }^{7} \mathrm{Be} \rightarrow{ }^{3} \mathrm{He}+{ }^{4} \mathrm{He}$ é de aproximadamente $1,59 \mathrm{MeV}$, baixa o suficiente para que o ${ }^{7} \mathrm{Be}$ possa ser tratado como um problema de dois corpos [2].

O primeiro estado excitado do núcleo ${ }^{7}$ Be possui baixa energia, dificultando a

\footnotetext{
${ }^{2}$ Elementos que possuem núcleon de valência, como ${ }^{6} \mathrm{He},{ }^{8} \mathrm{~B},{ }^{11} \mathrm{Be}$ e ${ }^{17} \mathrm{Ne}$.
} 
separação dos eventos relacionados ao espalhamento elástico e inelástico. Por isso, comumente nomeia-se a reação estudada como espalhamento quasielástico ${ }^{3}$.

Na literatura podem ser encontradas algumas medidas relacionadas a esse espalhamento: Verma et al [1] mediram esse espalhamento nas energias de $\mathrm{E}_{\text {lab }}=17,19$ e $21 \mathrm{MeV}$ e Mukha et al [2] mediram esse espalhamento em $\mathrm{E}_{\text {lab }}=23,7 \mathrm{MeV}$. Nesse trabalho propomos estudar essa reação na energia de $23,1 \mathrm{MeV}$. A escolha dessa energia foi feita levando em consideração o fato de que os dados de Mukha et al não estão normalizados, necessitando uma confirmação para a análise nesses dados [2].

Neste trabalho, utilizou-se a reação de produção ${ }^{7} \mathrm{Li}\left({ }^{6} \mathrm{Li},{ }^{7} \mathrm{Be}\right)$, através de um alvo de LiF.

A Tabela 1.2 mostra algumas características do núcleo ${ }^{7} \mathrm{Be}[17]$.

Tabela 1.2: Algumas características do núcleo ${ }^{7} \mathrm{Be}$.

Massa atômica $\quad 7,01 \mathrm{u}$

Tempo de meia vida $\quad 53,22 \mathrm{~d}$

Método de decaimento Captura de elétrons

Spin nuclear $\quad(3 / 2)^{-}$

Esse trabalho está estruturado da seguinte forma:

Capítulo 2 - Descrição Experimental: Detalhamento do aparato utilizado e procedimentos realizados para a execução do experimento;

Capítulo 3 - Experimento ${ }^{7} \mathbf{B e}+{ }^{9} \mathrm{Be}$ : Detalhes da realização do experimento e resultados obtidos;

Capítulo 4 - Fundamentos Teóricos: Informações teóricas relevantes para o entendimento do experimento e dos resultados obtidos;

Capítulo 5 - Análise dos Dados: Comparação com modelos teóricos para obtenção do potencial que descreve esse sistema;

Capítulo 6 - Conclusões: Considerações finais e perspectivas futuras.

\footnotetext{
${ }^{3}$ Alguns autores definem processos quasielásticos como a soma de todos os processos com exceção da fusão
} 
Introdução 


\section{Capítulo 2}

\section{Descrição Experimental}

A experiência proposta neste trabalho foi realizada utilizando o acelerador de partículas localizado no Instituto de Física da Universidade de São Paulo e o sistema RIBRAS para a seleção e focalização do feixe radioativo de interesse. No presente capítulo será feita a descrição do aparato experimental e da montagem utilizada para a realização das medidas do espalhamento ${ }^{7} \mathrm{Be}+{ }^{9} \mathrm{Be}$.

\section{$2.1 \quad$ Fonte SNICS}

Para a produção do feixe secundário radioativo de ${ }^{7} \mathrm{Be}$ foi utilizado um feixe primário de ${ }^{6} \mathrm{Li}$, produzido por uma fonte do tipo MC-SNICS (Multi Cathode Source of Negative Ion by Cesium Sputtering), utilizada no acelerador Pelletron.

A fonte MC-SNICS é composta por uma câmara a vácuo, acoplada a um reservatório de Césio (Cs), e um disco multi-cátodo, que possibilita a utilização de até 32 diferentes tipos de feixes sem a necessidade de quebra de vácuo, e, também mantém a fonte em operação. Dentro da câmara existem dois ionizadores de formato cônico e a entrada por onde o cátodo resfriado é colocado. Dentro dos cátodos existem pastilhas (cadinhos), confeccionadas geralmente de sais misturado com o elemento com o qual se quer produzir o feixe. No presente experimento foi utilizada uma pastilha de ${ }^{6} \mathrm{Li}_{2} \mathrm{O}+\mathrm{Ag}$ em proporção volumétrica de aproximadamente $50 \%$ de cada material. O esquema da fonte pode ser visto na Fig. 2.1.

Ao aquecer o reservatório, o Cs vaporizado deposita-se na superfície do cátodo 


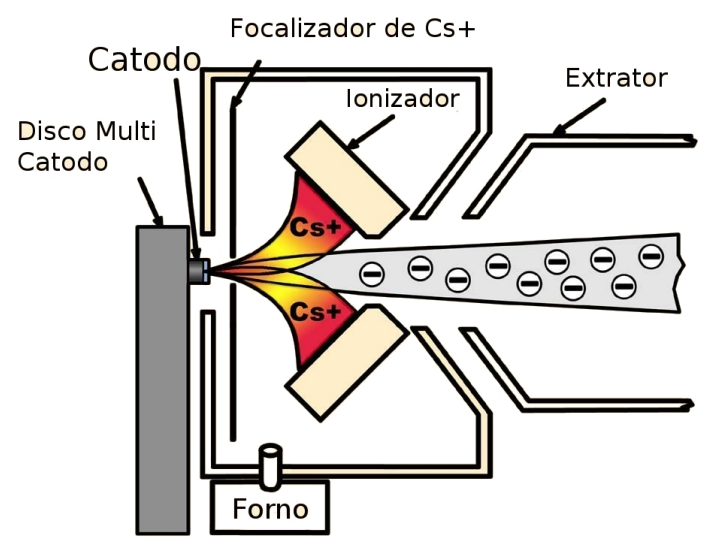

Figura 2.1: Esquema de funcionamento da fonte MC-SNICS [18].

resfriado, formando uma fina camada. Parte do vapor de Cs segue em direção ao ionizador, que tem a função de retirar elétrons do átomo, formando, dessa forma, $\mathrm{Cs}^{+}$. $\mathrm{O} \mathrm{Cs}^{+}$é atraído pelo potencial negativo do cátodo e, ao chocar-se com o cadinho, arranca átomos de diferentes elementos da pastilha, processo conhecido como sputtering. Esses átomos recebem elétrons ao atravessarem a fina camada de Cs formada, transição que ocorre devido ao fato deste ser um elemento muito eletropositivo. Desta forma esses átomos carregados negativamente serão repelidos pelo próprio cátodo e direcionados ao extrator, que os acelera eletrostaticamente por uma tensão de $90 \mathrm{kV}$ (a fonte de íons opera sob uma tensão de $20 \mathrm{kV}$ ). No processo de sputtering a corrente extraída é dada por:

$$
I=I^{+} S \eta
$$

onde $I^{+}$é a corrente de $\mathrm{Cs}^{+}$produzida pelo ionizador, $S$ é fração de íons que são emitidos pelo cátodo e $\eta$ é a probabilidade de que um átomo passando pela fina camada de Cs depositada sobre a saída do cátodo receba elétrons.

O feixe, ao ser produzido pela fonte de íons, contém diversos tipos de elementos com diferentes estados de carga. É possível selecionar o elemento desejado através de um eletroímã (ME-20), que deflete o feixe em $90^{\circ}$, selecionando os íons conforme a relação: 


$$
\xi=\frac{m E}{Z^{2}}
$$

onde $m$ é a massa, $E$ a energia e $Z$ o número atômico do íon ${ }^{1}$. O feixe selecionado é então injetado verticalmente no tubo do acelerador.

\section{$2.2 \quad$ Acelerador Pelletron}

O Pelletron é um acelerador eletrostático do tipo Tandem, produzido pela NEC e instalado no edifício Oscar Sala do IFUSP no ano de 1972 [19]. Com tensão máxima de $8 \mathrm{MV}$, o acelerador pode produzir feixes com energia até $5 \mathrm{MeV} / \mathrm{A}$.

A Fig. 2.2 mostra o esquema do acelerador, onde pode ser visto o corpo do acelerador, além da fonte de íons e a área experimental.

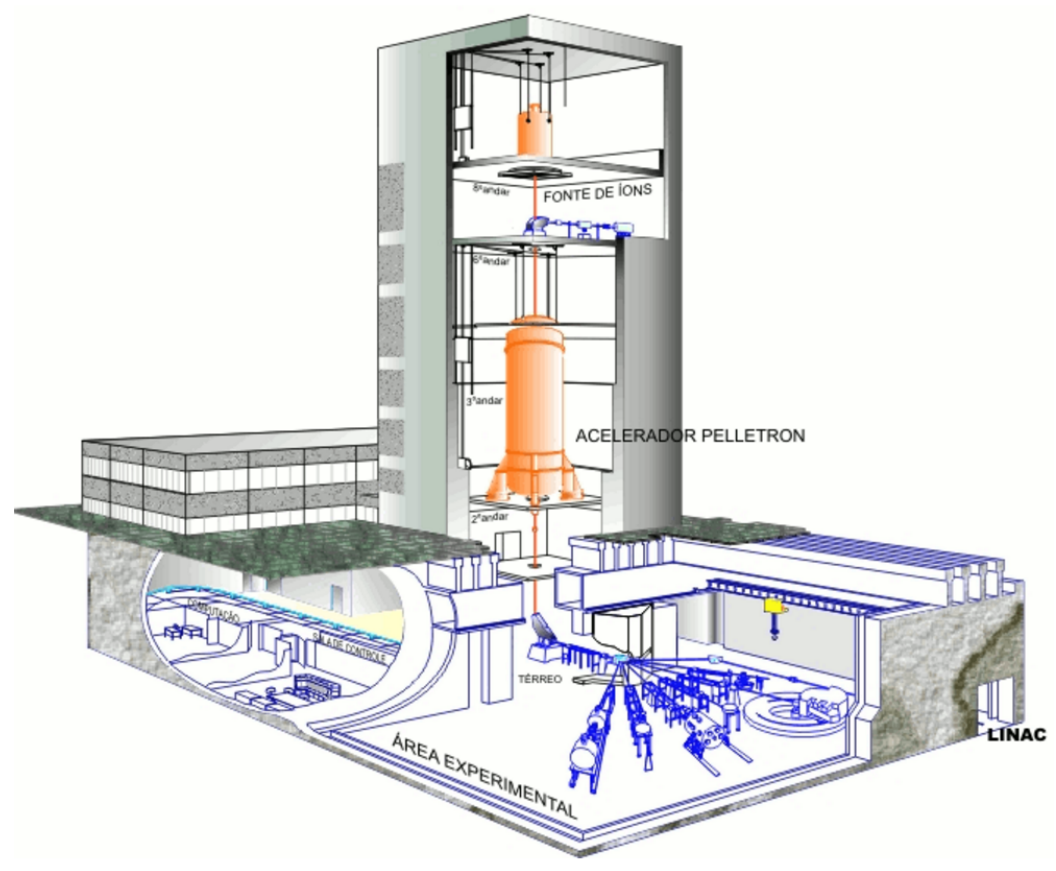

Figura 2.2: Esquema do acelerador Pelletron localizado no edifício Oscar Sala [20].

O feixe é acelerado em duas etapas principais:

\footnotetext{
${ }^{1} \mathrm{O}$ máximo valor que essa relação pode alcançar é $\xi=20$, valor que dá ao eletroímã o nome de ME-20.
} 
1. O feixe negativo, produzido pela fonte de íons, é atraído pelo potencial positivo do terminal de alta tensão do acelerador, localizado no centro do acelerador. Nessa etapa, o feixe atravessa uma folha de Carbono (stripper) que retira elétrons dos átomos, deixando-o carregado positivamente;

2. O feixe eletricamente positivo é repelido devido à tensão positiva do terminal. Desta forma, ao fim do processo de aceleração a energia do feixe é dada pela expressão:

$$
E_{f}=e\left(V_{e}+V_{p}\right)+(q+1) e V_{t}
$$

onde $e$ é a carga do elétron, $V_{e}$ é o potencial de extração da fonte de íons, $V_{p}$ é o potencial de pré-aceleração, $q$ é o estado de carga do feixe e $V_{t}$ é a tensão do terminal.

O método para a obtenção de altas tensões em um acelerador eletrostático se baseia no princípio de indução elétrica, sendo este uma evolução do gerador Van de Graff. Nesse acelerador uma cinta feita de nylon possui peças metálicas isoladas (pellets) que, ao passarem por um indutor, são carregadas positivamente e conduzidas pela cinta até o terminal do acelerador, carregando-o positivamente. Esse processo é capaz de produzir uma tensão muito estável no terminal. A Fig. 2.3 mostra um esquema desse método.

Como o feixe é produzido com diversos estados de carga em sua composição, ao término da aceleração este possuirá diferentes energias. Desta forma, um eletroímã (ME-200) é utilizado para realizar uma seleção em energia, segundo a Eq. (2.4) [22]:

$$
E=m c^{2}\left(\sqrt{1+\frac{2(q f c)^{2} k}{m c^{2}}}-1\right)
$$

onde $m$ é a massa de repouso do íon, $c$ é a velocidade da luz, $q$ é o estado de carga do feixe, $f$ é a frequência de ressonância do eletroímã e $k$ é a constante de calibração do ME-200. 


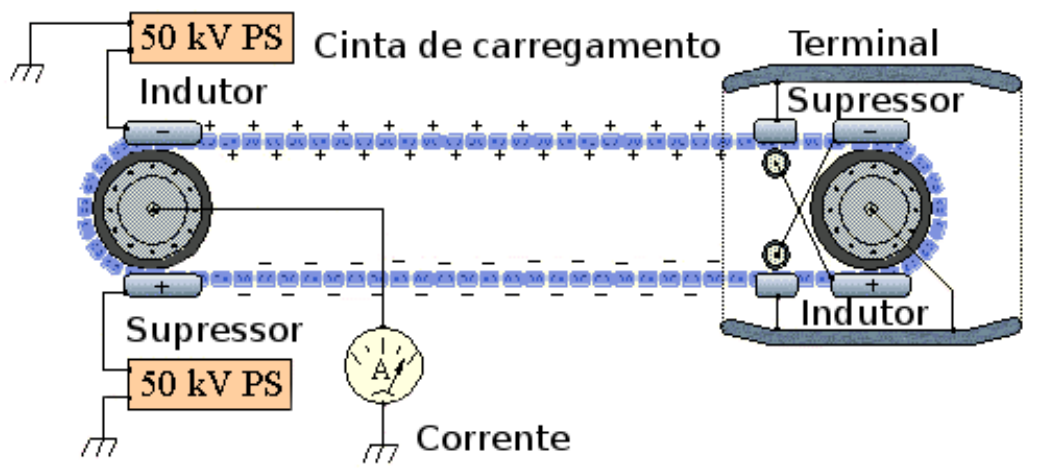

Figura 2.3: Esquema da geração de alta tensão no acelerador Pelletron (adaptado de $[21])$.

Além disso, o ME-200 tem a função de defletir em $90^{\circ}$ o feixe selecionado, que é então direcionado para a canalização desejada pelo switching magnet.

Ao longo de todo o tubo do acelerador existem quadrupolos elétricos e magnéticos para que o feixe seja constantemente alinhado e focalizado. A intensidade do feixe pode ser medida utilizando copos de Faraday, que estão instalados em diferentes posições do acelerador. A Fig. 2.4 apresenta um esquema da estrutura do acelerador. 


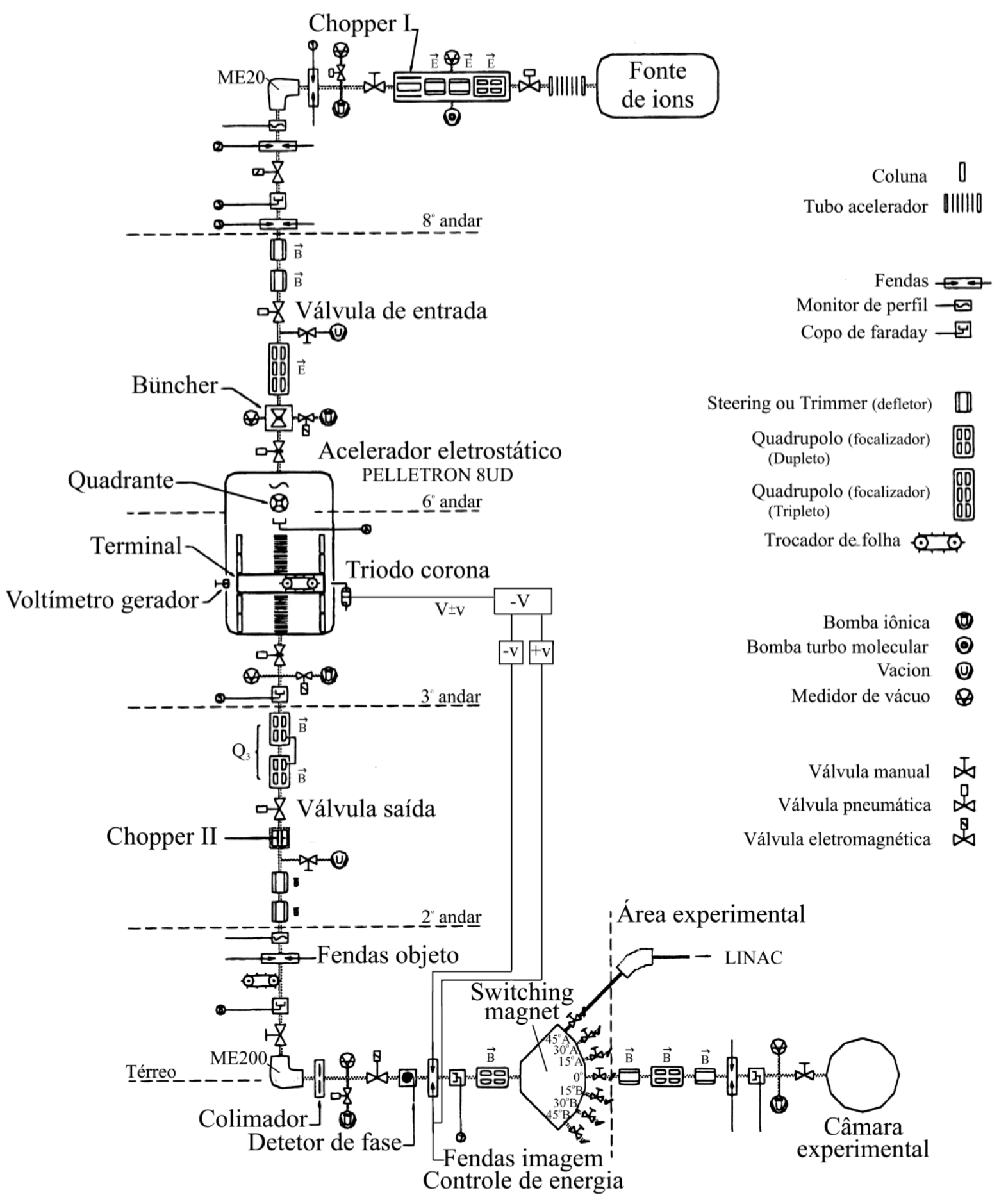

Figura 2.4: Esquema do acelerador eletrostático do laboratório Pelletron. Imagem de J.C. Terassi. 


\subsection{Sistema RIBRAS}

O sistema RIBRAS [13, 14] (Radioactive Ion Beams in Brasil) é formado por dois solenoides supercondutores que são utilizados para selecionar e focalizar núcleos fora da linha de estabilidade. Esse sistema, instalado na canalização a $45^{\circ}$ da sala experimental B do Laboratório Aberto de Física Nuclear do IFUSP, entrou em funcionamento em 2004 utilizando apenas um solenoide, apenas em 2011 começaram as experiências com ambos solenoides. Os solenoides foram fabricados pela empresa Cryomagnetics Inc.. A Fig. 2.5 mostra um esquema do Sistema RIBRAS.

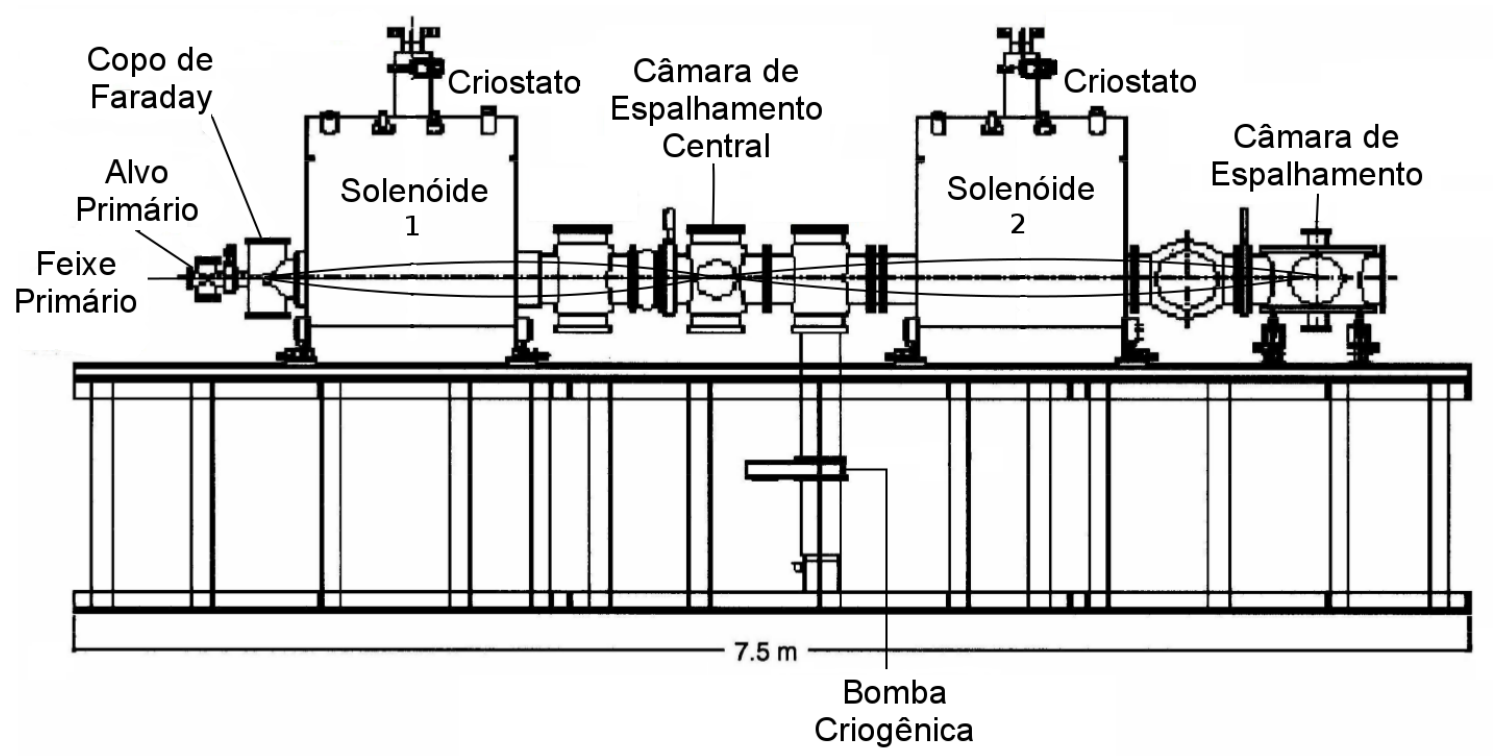

Figura 2.5: Esquema do sistema RIBRAS [5].

O feixe secundário é produzido através da interação entre o feixe primário, proveniente do acelerador Pelletron, e o alvo primário, localizado antes do primeiro solenoide (ver Fig. 2.5). O alvo primário consiste em uma pequena câmara com janelas que permitem a entrada e saída do feixe primário. A distância entre a janela de entrada e de saída é de 3,6 cm. Nesse sistema há a possibilidade de utilizar alvos gasosos e, neste caso, as janelas são fechadas com, por exemplo, folhas de havar.

Após o alvo primário há um copo de Faraday posicionado a zero grau, utilizado para interceptar o feixe primário e medir sua intensidade. Devido à presença do copo de Faraday e o uso de colimadores para bloquear partículas indesejadas, o ângulo 
de aceitação do solenoide é de 2 a 6 graus. A Fig. 2.6 mostra o esquema do alvo primário do sistema RIBRAS.

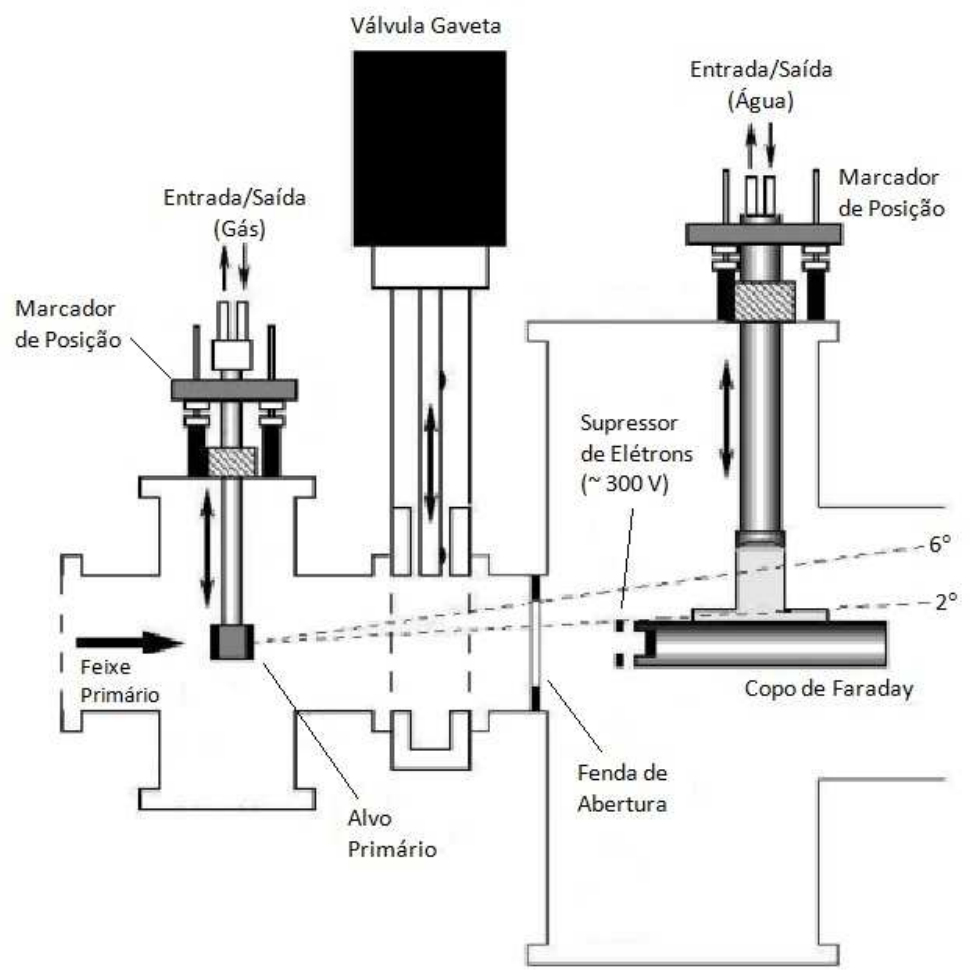

Figura 2.6: Esquema mostrando o alvo primário e o copo de Faraday [5].

As reações de produção em baixas energias possuem baixas seções de choque e, portanto, a intensidade do feixe secundário é relativamente baixa. Apesar disso, o RIBRAS é muito bem sucedido na produção e utilização de feixes instáveis como por exemplo ${ }^{8} \mathrm{Li},{ }^{6} \mathrm{He},{ }^{7,10} \mathrm{Be}$, entre outros. Algumas reações de produção dos feixes secundários e suas respectivas intensidades, obtidas com o sistema RIBRAS, são exemplificadas na Tabela 2.1.

A bobina interna dos solenoides, responsável pelo campo magnético, está mergulhada em Hélio (He) líquido a uma temperatura de 4K. Esse reservatório de He é isolado termicamente por duas camadas, sendo uma de vácuo e outra de Nitrogênio (N) líquido que deve ser mantido em um certo nível para que o He não evapore rapidamente.

Os solenoides são capazes de selecionar o feixe de interesse entre muitos que são produzidos na reação de produção entre o feixe primário e o alvo primário. A 
Tabela 2.1: Algumas reações utilizadas para produção de feixes no sistema RIBRAS.

\begin{tabular}{ccc}
\hline Feixe Secundário & Reação & Intensidade $(\mathrm{pps})$ \\
\hline${ }^{6} \mathrm{He}$ & ${ }^{9} \mathrm{Be}\left({ }^{6} \mathrm{Li},{ }^{6} \mathrm{He}\right)$ & $10^{5}$ \\
${ }^{6} \mathrm{He}$ & ${ }^{9} \mathrm{Be}\left({ }^{7} \mathrm{Li},{ }^{6} \mathrm{He}\right)$ & $10^{5}$ \\
${ }^{8} \mathrm{Li}$ & ${ }^{9} \mathrm{Be}\left({ }^{7} \mathrm{Li},{ }^{8} \mathrm{Li}\right)$ & $10^{6}$ \\
${ }^{7} \mathrm{Be}$ & ${ }^{3} \mathrm{He}\left({ }^{7} \mathrm{Li},{ }^{7} \mathrm{Be}\right)$ & $10^{5}$ \\
${ }^{7} \mathrm{Be}$ & ${ }^{3} \mathrm{He}\left({ }^{6} \mathrm{Li},{ }^{7} \mathrm{Be}\right)$ & $10^{5}$ \\
${ }^{8} \mathrm{~B}$ & ${ }^{3} \mathrm{He}\left({ }^{6} \mathrm{Li},{ }^{8} \mathrm{~B}\right)$ & $10^{4}$ \\
${ }^{18 m} \mathrm{~F}$ & ${ }^{3} \mathrm{He}\left({ }^{18} \mathrm{O},{ }^{18 m} \mathrm{~F}\right)$ & $10^{4}$ \\
\hline
\end{tabular}

seleção se faz pelo campo magnético $(\vec{B})$, produzido pelo solenoide, que distingue os elementos que possuem a mesma rigidez magnética (ver Eq. (2.9)). O campo magnético no interior do solenoide interage com as partículas carregadas em voo, produzindo a força de Lorentz na forma:

$$
\vec{F}_{m a g}=q \vec{v} \wedge \vec{B}
$$

onde $q$ é a carga e $\vec{v}$ a velocidade da partícula carregada incidente no campo magnético $\vec{B}$. A força de Lorentz tem a propriedade de ser perpendicular à direção de propagação da partícula carregada $(\vec{v})$, sendo uma resultante centrípeta e curvando o feixe segundo a equação:

$$
q v B=\frac{m v^{2}}{\rho}
$$

onde $\rho$ é o raio de curvatura da trajetória e $m$ é a massa de repouso. Define-se a rigidez magnética como:

$$
B \rho=\frac{m v}{q}
$$

Pode-se escrever a energia da seguinte forma: 


$$
E=\frac{p^{2}}{2 m}
$$

onde $p=m v$ e E é a energia da partícula.

Unindo as Eqs. 2.7 e 2.8, obtém-se a expressão matemática que coordena a seleção dos feixes carregados no campo magnético do solenoide:

$$
B \rho=\frac{\sqrt{2 m E}}{q}
$$

Para bloquear a passagem de partículas que possuem rigidez magnética diferente do valor referente ao feixe de interesse são utilizados colimadores e bloqueadores, estrategicamente posicionados conforme pode ser visto na Fig. 2.7.

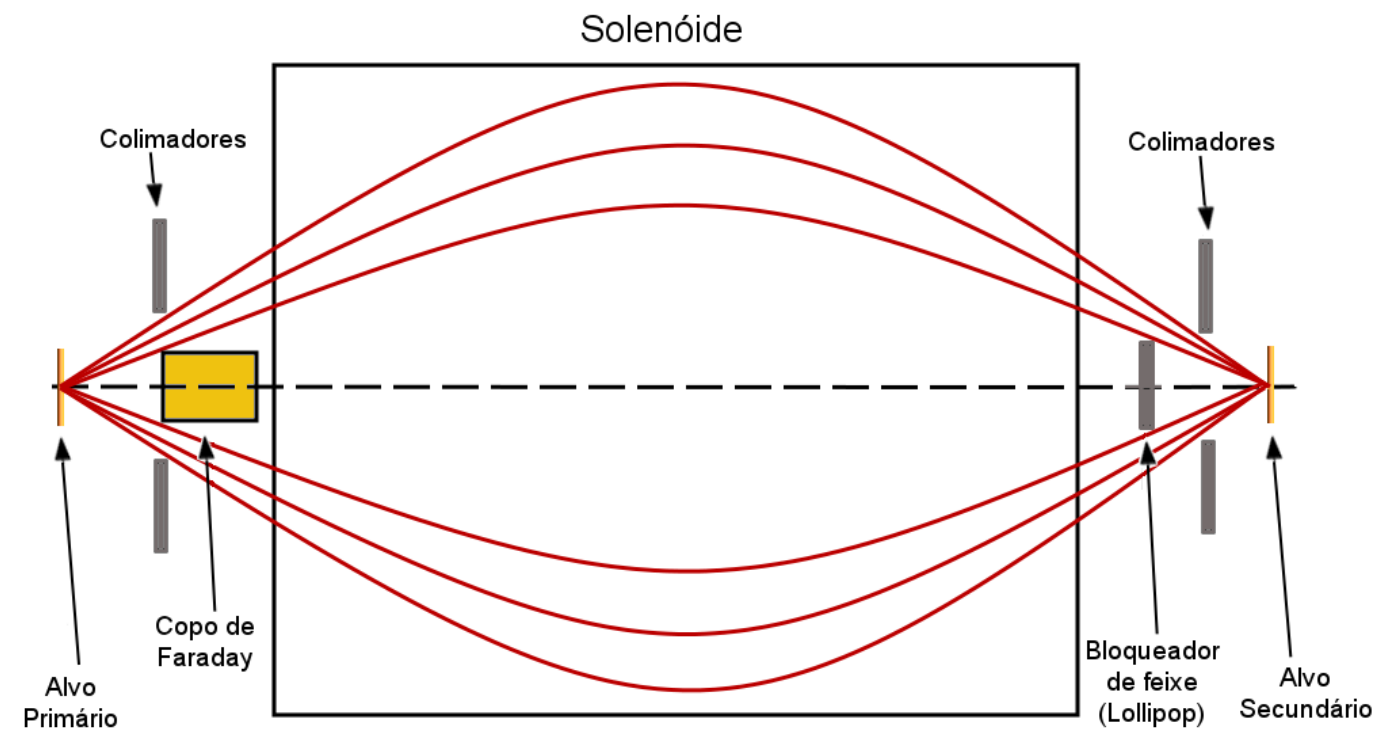

Figura 2.7: Exemplo de esquema dos colimadores e bloqueadores posicionados próximos do primeiro solenoide.

Alguns feixes secundários possuem rigidez magnética próxima do valor referente ao feixe de interesse sendo, portanto, focalizados e conhecidos como contaminantes. Esse problema pode ser parcialmente resolvido com a utilização dos dois solenoides, juntamente com um degradador na câmara central. O degradador tem como obje- 
tivo fazer com que os feixes selecionados pelo primeiro solenoide percam diferentes quantidades de energia, de tal forma que o segundo solenoide consiga separá-los utilizando o mesmo princípio de rigidez magnética.

\subsubsection{Câmara de Espalhamento e Sistema de Detecção}

A medida do espalhamento ${ }^{7} \mathrm{Be}+{ }^{9} \mathrm{Be}$ foi realizada na câmara de espalhamento central, que possui diâmetro de $25 \mathrm{~cm}$, um suporte para o posicionamento dos alvos e um prato giratório, que permite a movimentação dos detectores sem que haja a necessidade de desmontar o aparato e quebrar o vácuo do sistema (ver Fig. 2.8).

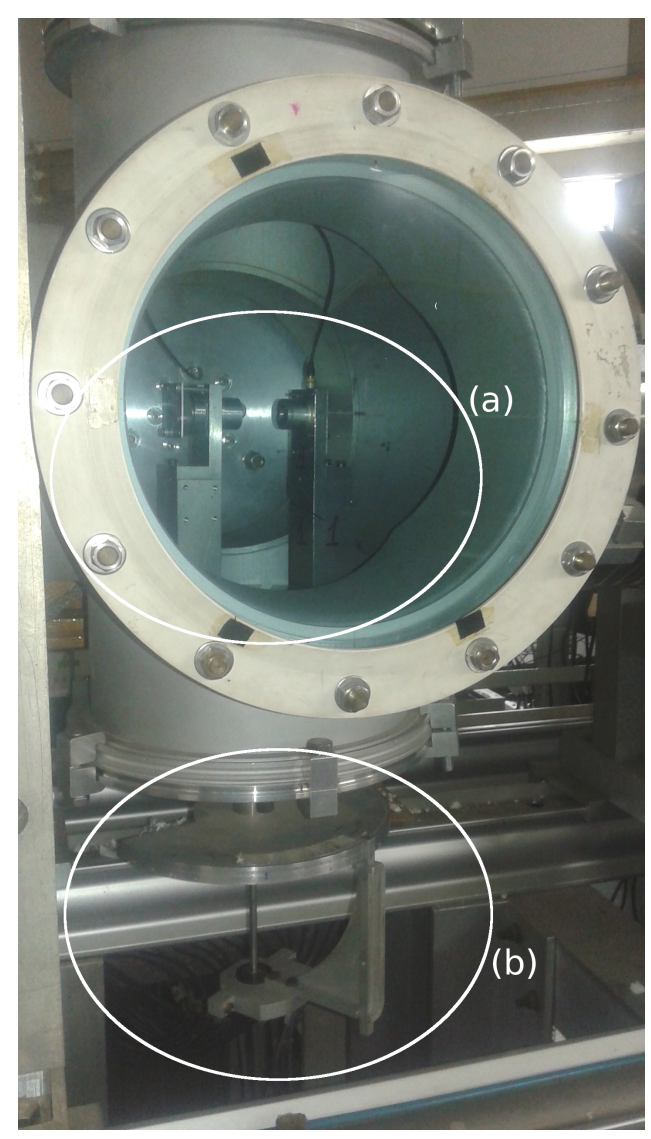

Figura 2.8: Foto da câmara central, mostrando (a) dois telescópios montados e (b) a haste para movimentação do prato giratório.

Foram montados dois telescópios $(\mathrm{E}-\Delta \mathrm{E})$ e dois detectores $(\mathrm{E})$ de barreira de superfície de Silício (Si). O princípio de funcionamento desses detectores é baseado no conceito de perda de energia, onde a energia depositada pela interação de uma 
partícula com o meio depende da massa $(m)$, número atômico $(Z)$ e energia da partícula $(E)$, fazendo que diferentes partículas percam diferentes quantidades de energia ao atravessar o detector, conforme a equação [23]:

$$
\frac{\Delta E}{\Delta x} \propto \frac{m Z^{2}}{E}
$$

A interação das partículas com o meio material dos detectores libera, em geral, elétrons, que são capturados por uma diferença de potencial aplicada no detector (semicondutor), produzindo uma corrente e uma carga total que é proporcional à energia inicial da partícula. Desta forma, é possível identificar as partículas que foram detectadas.

A Tabela 2.2 mostra as especificações dos detectores utilizados neste trabalho.

Tabela 2.2: Especificações dos detectores utilizados.

\begin{tabular}{lcc}
\hline Detector & Espessura $(\mu \mathrm{m})$ & Tensão Utilizada $(\mathrm{V})$ \\
\hline $\mathrm{E}_{1}$ & 150 & 70 \\
$\Delta \mathrm{E}_{2}$ & 25 & 40 \\
$\mathrm{E}_{2}$ & 150 & 70 \\
$\Delta \mathrm{E}_{3}$ & 25 & 40 \\
$\mathrm{E}_{3}$ & 500 & 170 \\
$\mathrm{E}_{4}$ & 150 & 70 \\
\hline
\end{tabular}

Uma técnica muito útil quando se trabalha com contaminantes de massa e energia próximas às da partícula de interesse, é considerar as diferentes perdas de energia de uma partícula em um telescópio $(\Delta \mathrm{E}-\mathrm{E})$. Com isso, espectros biparamétricos $(\Delta \mathrm{E}$ x E) são construídos de forma a identificar as partículas com relativa facilidade, já que desta forma é possível diferenciar os picos.

Os detectores $\Delta \mathrm{E}$-E foram montados em suportes e fixados no prato giratório da câmara de espalhamento central, conforme esquema apresentado na Fig. 2.9(a). Os telescópios foram posicionados com $60^{\circ}$ de espaçamento entre si e os detectores com $30^{\circ}$ em relação aos telescópios. Foram utilizados colimadores no suporte dos 
telescópios (ver Fig. 2.9(b)) para que o ângulo de aceitação não fosse muito grande e a intensidade do feixe incidente não prejudicasse os detectores.
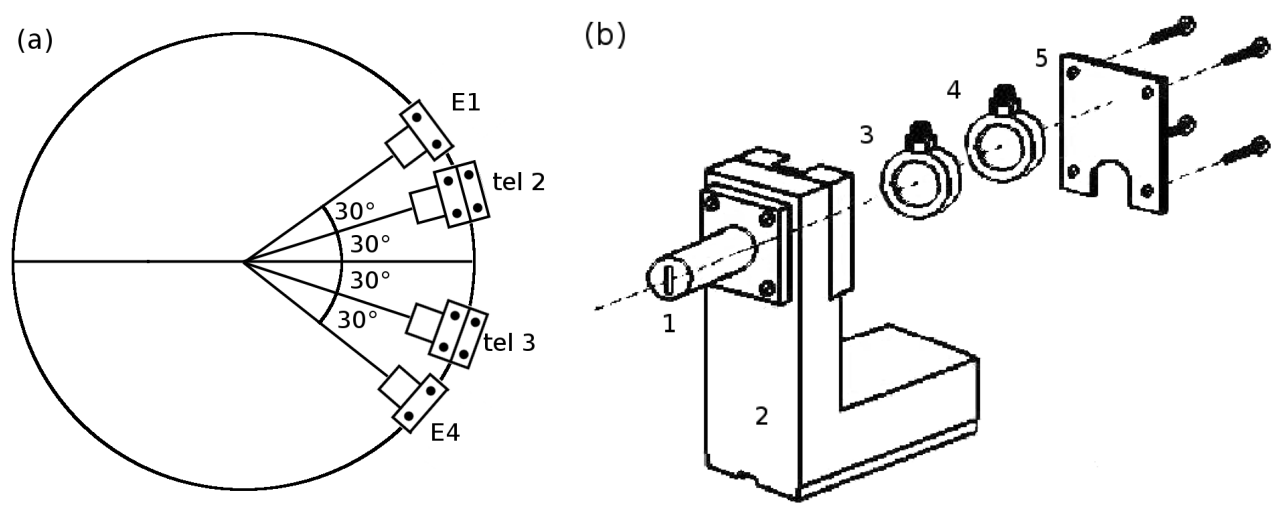

Figura 2.9: (a) Esquema da montagem dos detectores no prato giratório e (b) exemplo de um telescópio indicando o (1) colimador, (2) suporte, (3) detector $\Delta \mathrm{E},(4)$ detector E, (5) tampa de fixação [2].

Foram realizadas medidas na região angular compreendida entre $15^{\circ}-78^{\circ} \mathrm{com}$ passo de $3^{\circ}$ no sistema do laboratório. Um exemplo dos espectros mono e biparamétricos obtidos no experimento, é apresentado na Fig. 2.10.

O ângulo sólido do detector, pode ser obtido através da relação $\Delta \Omega=\frac{A}{d^{2}}[10]$, onde $A$ é a área de entrada do feixe de partículas no detector e $d$ a distância do centro do alvo secundário até a entrada do telescópio (colimador). A distância entre os detectores e o alvo e os valores dos ângulos sólidos obtidos para os detectores são apresentados na Tabela 2.3.

Tabela 2.3: Distâncias do alvo-detetor e ângulos sólidos obtidos ${ }^{1}$.

\begin{tabular}{lcc}
\hline Detector & Distância alvo-detector $(\mathrm{cm})$ & $\Delta \Omega(\mathrm{msr})$ \\
\hline $\mathrm{E}_{1}$ & $7,82(5)$ & $24,5(1)$ \\
$\Delta \mathrm{E}_{2}-\mathrm{E}_{2}$ & $7,81(5)$ & $24,6(1)$ \\
$\Delta \mathrm{E}_{3}-\mathrm{E}_{3}$ & $7,84(5)$ & $24,4(1)$ \\
$\mathrm{E}_{4}$ & $8,05(5)$ & $23,1(1)$ \\
\hline
\end{tabular}

\footnotetext{
${ }^{1}$ Os erros apresentados foram calculados levando em consideração as incertezas da geometria utilizada (áreas e distâncias).
} 

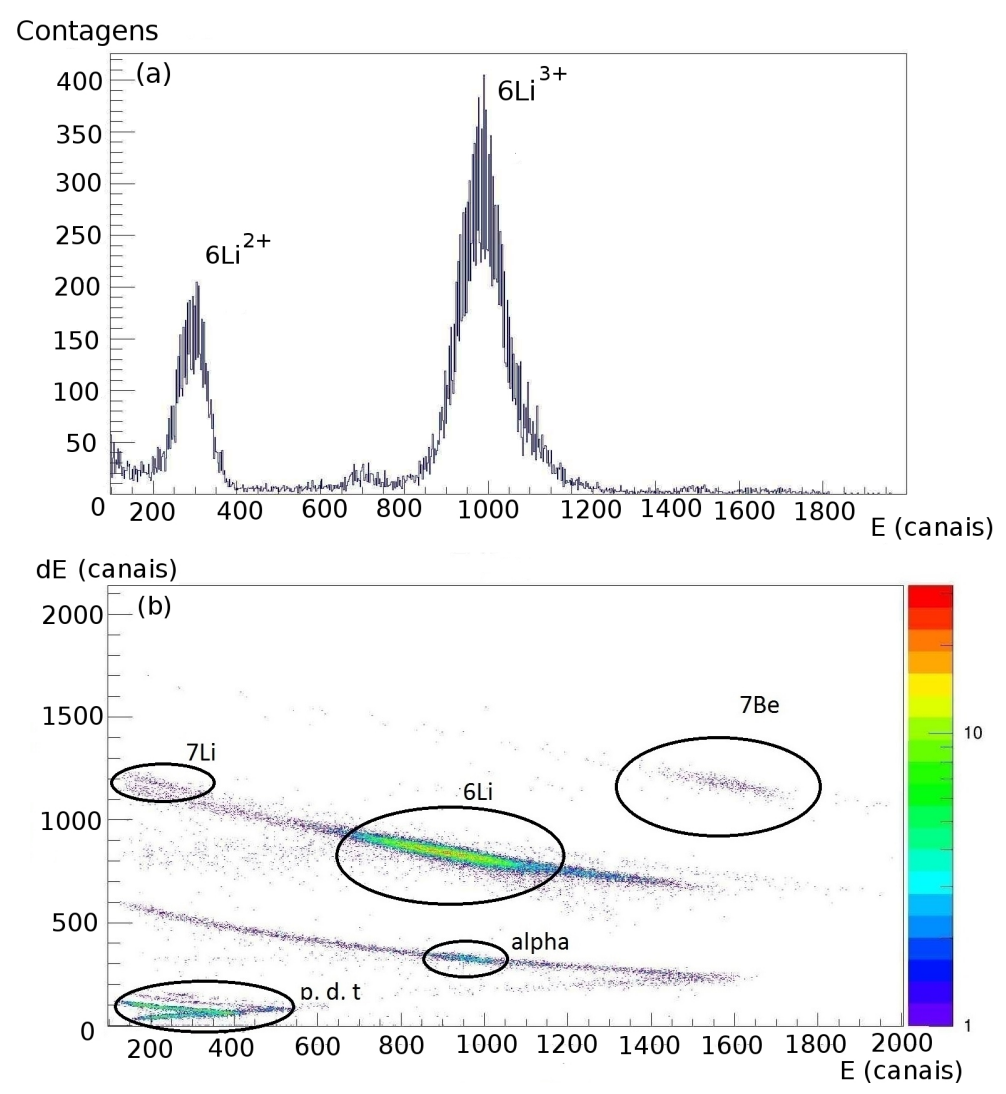

Figura 2.10: Exemplos de espectros (a) mono e (b) biparamétricos obtidos no experimento para o espalhamento quasielástico ${ }^{7} \mathrm{Be}+{ }^{9} \mathrm{Be}$.

\subsubsection{Sistema de Aquisição}

Os detectores produzem pulsos de carga que precisam ser processados para o seu armazenamento no computador. Esse processamento é feito por uma sequência de módulos eletrônicos. Primeiramente, os pulsos provenientes dos detectores passam por pré-amplificadores, que possuem duas saídas: uma de tempo e outra de energia.

O sinal de energia é enviado aos módulos amplificadores, onde são verificadas e, se necessário, ajustadas as alturas e formas dos pulsos. Esses sinais são enviados diretamente ao CAMAC (Computer Automated Measurement and Control), onde são convertidos em sinais digitais que serão enviados ao computador.

Os sinais de tempo, por sua vez, são enviados a um amplificador rápido e, em seguida, a um CFD (Constant Fraction Discriminator) que produz um sinal lógico chamado de gate. Os sinais de cada detector ( $\Delta \mathrm{E}$ ou $\mathrm{E})$ são direcionados ao módulo Fan-in/Fan-out, que produz um único sinal para todos os detectores. Esse sinal é 
enviado ao módulo Gate and Delay Generator, que produz o sinal utilizado pelo CAMAC para definir a chegada de um evento.

Os dois sinais, energia e tempo, são enviados ao CAMAC, que usa o sinal de gate (tempo) para integrar a corrente do sinal de energia e obter o valor da carga elétrica depositada pelo evento no detector. Esses sinais são, então, convertidos em sinais digitais e enviados ao microcomputador, que os armazena no Hard Drive. A Fig. 2.11 mostra o esquema da eletrônica utilizada.

No computador, os programas utilizados para receber os dados e criar os histogramas foram o SPM-ROOT e o SCANROOT [24], respectivamente.

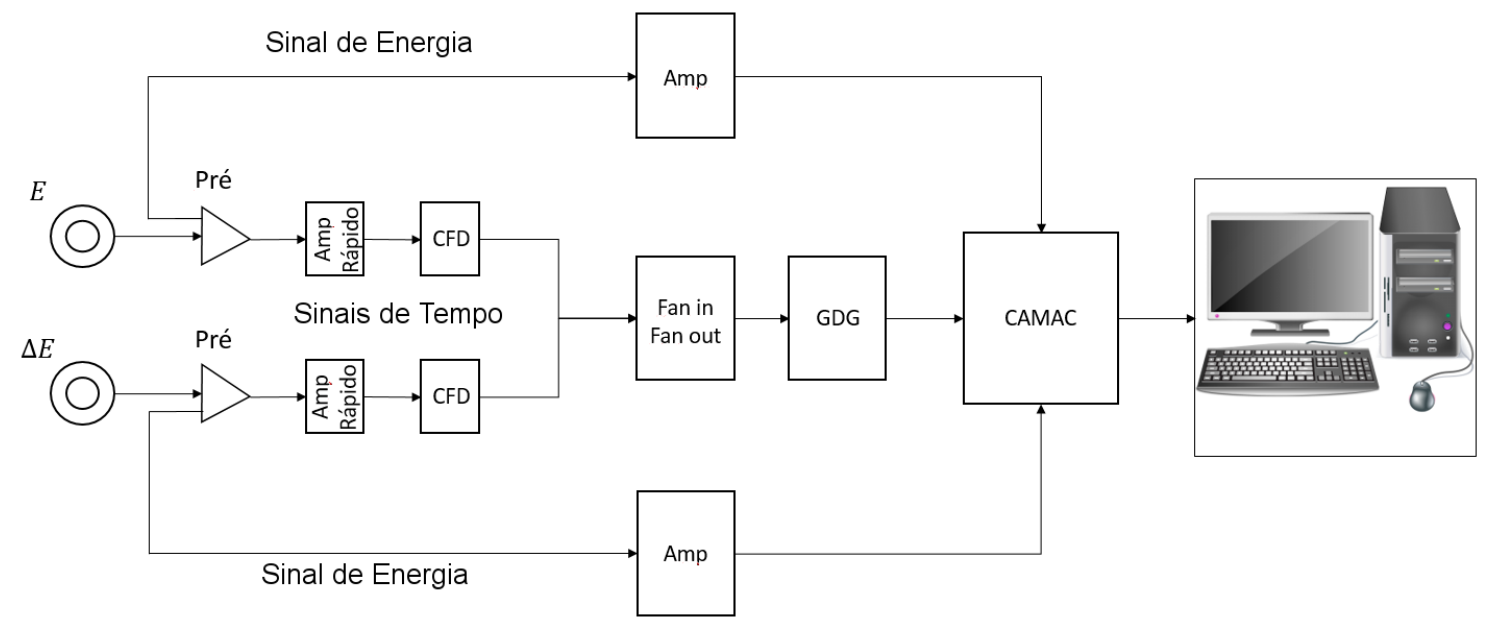

Figura 2.11: Esquema da eletrônica utilizada no experimento realizado neste trabalho. 


\section{Capítulo 3}

\section{O Experimento ${ }^{7} \mathrm{Be}+{ }^{9} \mathrm{Be}$}

Nesse capítulo será apresentado o método utilizado para a redução dos dados $e$ os resultados obtidos para o espalhamento quasielástico ${ }^{7} \mathrm{Be}+{ }^{9} \mathrm{Be} e{ }^{7} \mathrm{Be}+{ }^{197} \mathrm{Au}$ nas energias de $E_{\mathrm{lab}}=23,1 \mathrm{MeV}$ e $E_{\mathrm{lab}}=23,3 \mathrm{MeV}$, respectivamente. Cálculos preliminares de cinemática e perdas de energia, importantes para o planejamento e boa execução do experimento, foram efetuados utilizando o pacote UPAK [25], onde encontram-se os programas STOPX (perda de energia) e KINEQ (cinemática da reação).

\subsection{Alvos}

Os alvos utilizados nesse trabalho consistem em folhas finas de ${ }^{9} \mathrm{Be}, \mathrm{LiF}$ e ${ }^{197} \mathrm{Au}$. Os alvos de $\mathrm{LiF}$ e ${ }^{197} \mathrm{Au}$ foram confeccionados no Laboratório de Alvos do Instituto de Física da Universidade de São Paulo. O alvo de LiF foi produzido através de uma técnica de vaporização conhecida como PVD (Physical Vapor Deposition) [26], onde o sal de LiF foi vaporizado sendo depositado sobre uma fina camada de ${ }^{27} \mathrm{Al}^{1}$, produzida pelo mesmo método, necessária devido ao fato de o sal não ser autoportante, ou seja, não se sustenta sem a presença de outro material. O alvo de ${ }^{197} \mathrm{Au}$ foi produzido através da técnica de prensagem. O alvo de ${ }^{9} \mathrm{Be}$ foi confeccionado utilizando uma folha fina e auto-portante, adquirida da empresa BrushwellmanEUA.

\footnotetext{
${ }^{1} \mathrm{O}{ }^{27} \mathrm{Al}$ foi escolhido por ser um metal de fácil manipulação e por ter baixo ponto de evaporação.
} 
As espessuras dos alvos de ${ }^{9} \mathrm{Be},{ }^{197} \mathrm{Au}$ e $\mathrm{LiF}$ foram aferidas na sala de medidas localizada no primeiro andar do edifício Oscar Sala, utilizando uma fonte alfa $\left({ }^{241} \mathrm{Am}\right)$ com energia de 5,486 MeV (Medida 1). Essa medida se baseia na perda de energia dessas partículas ao atravessar o alvo. A comparação das energias obtidas para a partícula alfa, quando esta atravessa o alvo e em uma medida sem o alvo, fornece a espessura do mesmo [5].

Foi realizada, também, a medida da espessura do alvo de ${ }^{197} \mathrm{Au}$ no laboratório LAMFI utilizando a técnica Rutherford Backscattering Spectrometry (RBS) [27] que baseia-se no retroespalhamento Rutherford (Medida 2). Neste método um feixe monoenergético incide sobre o alvo gerando diferentes perdas de energia, que são relativas à profundidade em que ocorreu o espalhamento, e com isso é possível estimar a espessura do alvo. Os resultados obtidos estão apresentados na Tabela 3.1. As incertezas obtidas levam em consideração a largura dos picos obtidos com os dois métodos.

Tabela 3.1: Espessuras dos alvos utilizados neste trabalho.

\begin{tabular}{lcc}
\hline Alvo & Medida $1\left(\mathrm{mg} / \mathrm{cm}^{2}\right)$ & Medida $2\left(\mathrm{mg} / \mathrm{cm}^{2}\right)$ \\
\hline${ }^{9} \mathrm{Be}$ & $1,9(1)$ & - \\
${ }^{197} \mathrm{Au}$ & $4,4(1)$ & $4,22(1)$ \\
$\mathrm{LiF}$ & $0,4(2)$ & - \\
\hline
\end{tabular}

Observando a Tabela 3.1 nota-se que os valores obtidos para a espessura do alvo de ${ }^{197} \mathrm{Au}$, utilizando ambos os métodos, são compatíveis dentro da barra de erro. Desta forma, e devido ao fato de termos as espessuras dos outros alvos unicamente pelo método $1^{2}$, optou-se por utilizar apenas os resultados obtidos por esse método em todo o trabalho.

\footnotetext{
${ }^{2} \mathrm{O}$ método de RBS não é eficaz em medir diferentes camadas de um alvo, como o alvo de LiF. Devido à sensibilidade do alvo de ${ }^{9} \mathrm{Be}$, optou-se por não realizar a medida no LAMFI com este alvo.
} 


\subsection{Taxa de Produção}

Medidas envolvendo núcleos radioativos têm baixa seção de choque de produção, por isso, a focalização do feixe de interesse no alvo secundário deve ser maximizada no início do experimento. A corrente do solenoide, que maximiza a incidência do feixe de interesse no alvo secundário, foi calculada numericamente através do código computacional TWSP, adaptado do programa TWINSOL [11] para o sistema RIBRAS por R. Lichtenthäler. O valor obtido através desse método foi de $I_{\text {sol }}=$ 23,285 A. A Fig.3.1 mostra um exemplo das trajetórias calculadas numericamente, onde a linha representa o feixe de ${ }^{7} \mathrm{Be}$ de interesse. Pode-se observar o modo que o conjunto de colimadores e bloqueadores reduzem os possíveis contaminantes com rigidez magnética diferente.

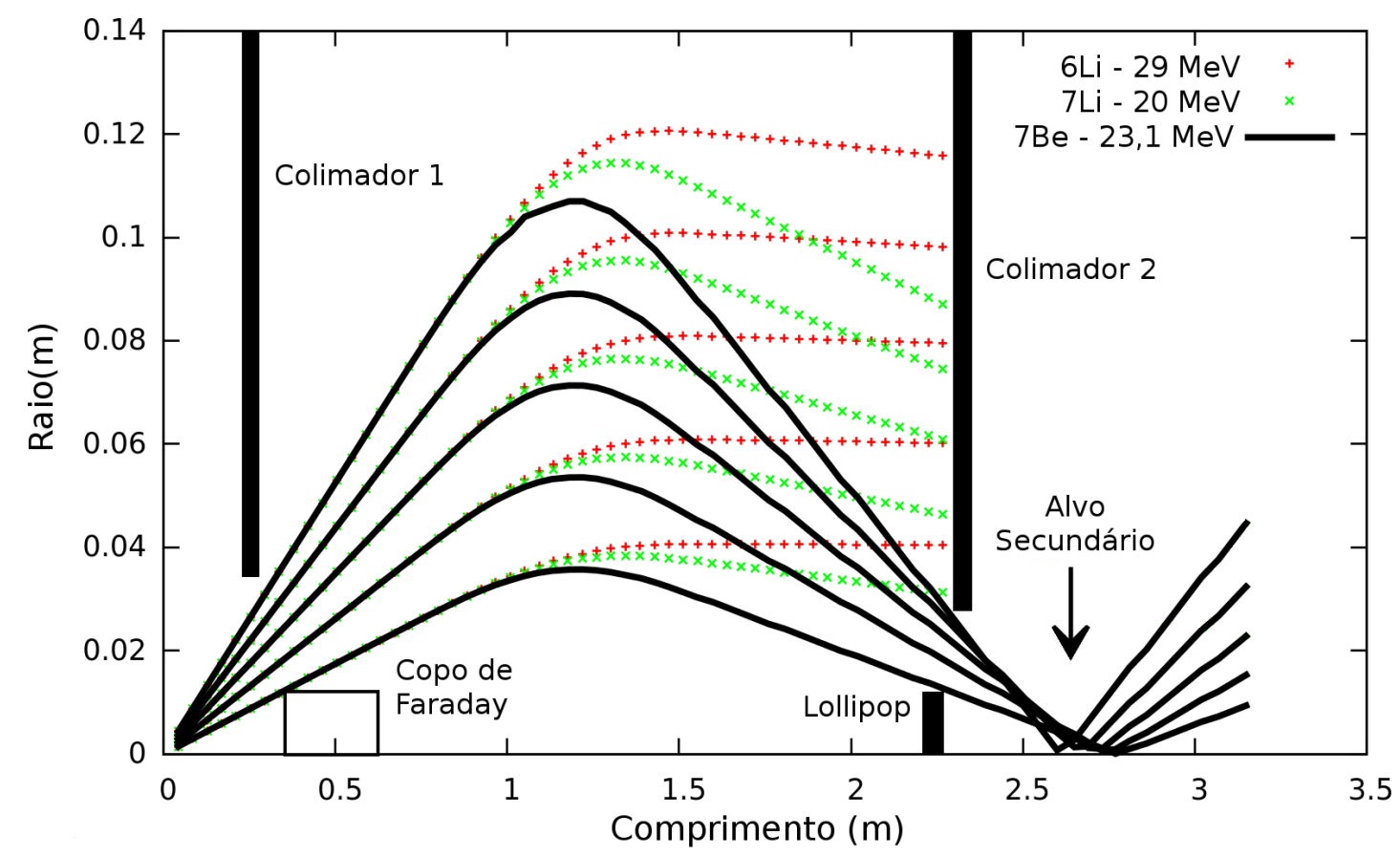

Figura 3.1: Trajetórias calculadas com o algoritmo TWSP [11].

É importante verificar experimentalmente o valor obtido através da simulação computacional. Para isso, varia-se a corrente do solenoide maximizando a razão:

$$
T=\frac{N_{7} B e}{I}
$$


onde $N_{7}$ Be é a área do pico de interesse ${ }^{7} B$ e e $I$ é corrente total do feixe primário de ${ }^{6} \mathrm{Li}$ integrada no tempo (integrador). Ao maximizar essa razão garante-se que o maior número possível de partículas de interesse atinjam o alvo secundário, aumentando o número de contagens da reação. A Fig. 3.2 mostra a variação dessa razão em função da corrente do solenoide obtida no experimento.

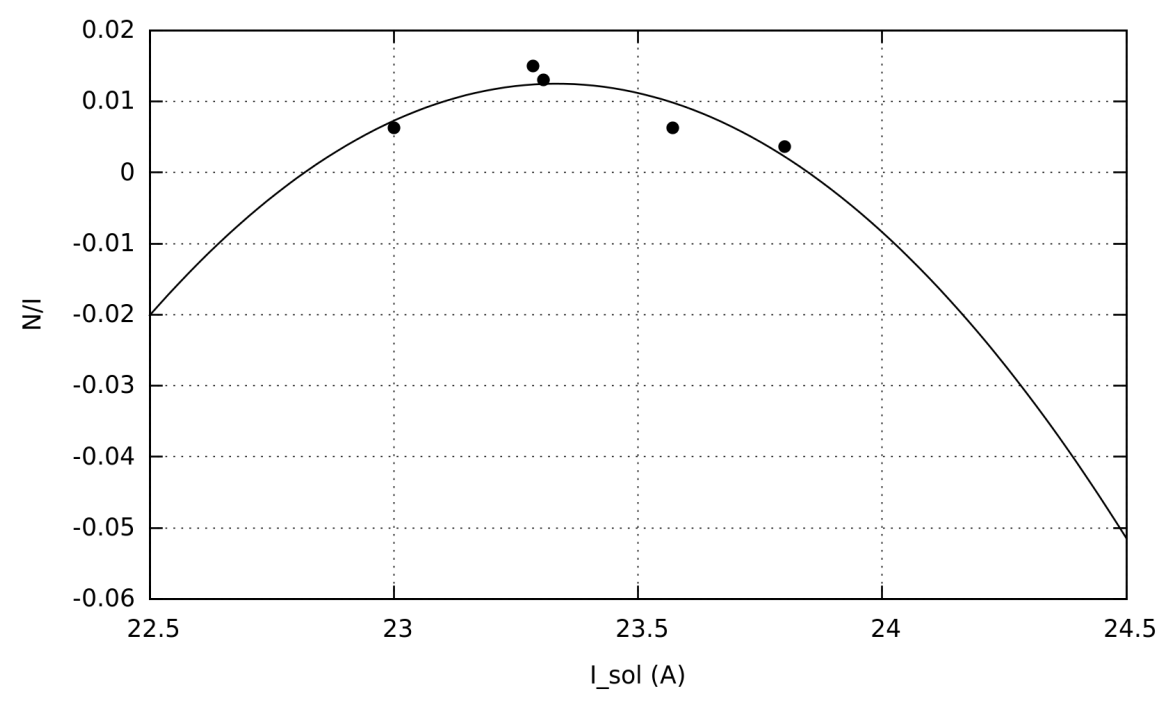

Figura 3.2: Gráfico da taxa de produção x corrente do solenoide.

O ajuste dos pontos forneceu a função:

$$
I_{\text {sol }}=-0,04(2) x^{2}+2(1) x-25(11)
$$

que tem ponto de máximo em $I_{\text {sol }}^{\max }=23,3 \mathrm{~A}$, igual ao valor de corrente do solenoide utilizado para a realização do experimento.

\subsection{Medidas do Espalhamento ${ }^{7} \mathrm{Be}+{ }^{9} \mathrm{Be}$}

Foram realizadas medidas de espalhamento do sistema ${ }^{7} \mathrm{Be}+{ }^{9} \mathrm{Be}$ para $\mathrm{E}_{\mathrm{lab}}=23,1$ $\mathrm{MeV}$. A energia da barreira Coulombiana no sistema do centro de massa é de $\mathrm{E}_{\mathrm{B}}=$ 4,44 MeV. Um exemplo típico do espectro biparamétrico obtido com alvo de ${ }^{9} \mathrm{Be} e$ 
${ }^{197} \mathrm{Au}$ é apresentado na Fig. 3.3, onde é possível ver os picos de ${ }^{7} \mathrm{Be},{ }^{9} \mathrm{Be},{ }^{6} \mathrm{Li},{ }^{7} \mathrm{Li}$, alfas, prótons, deutérios e trítios.
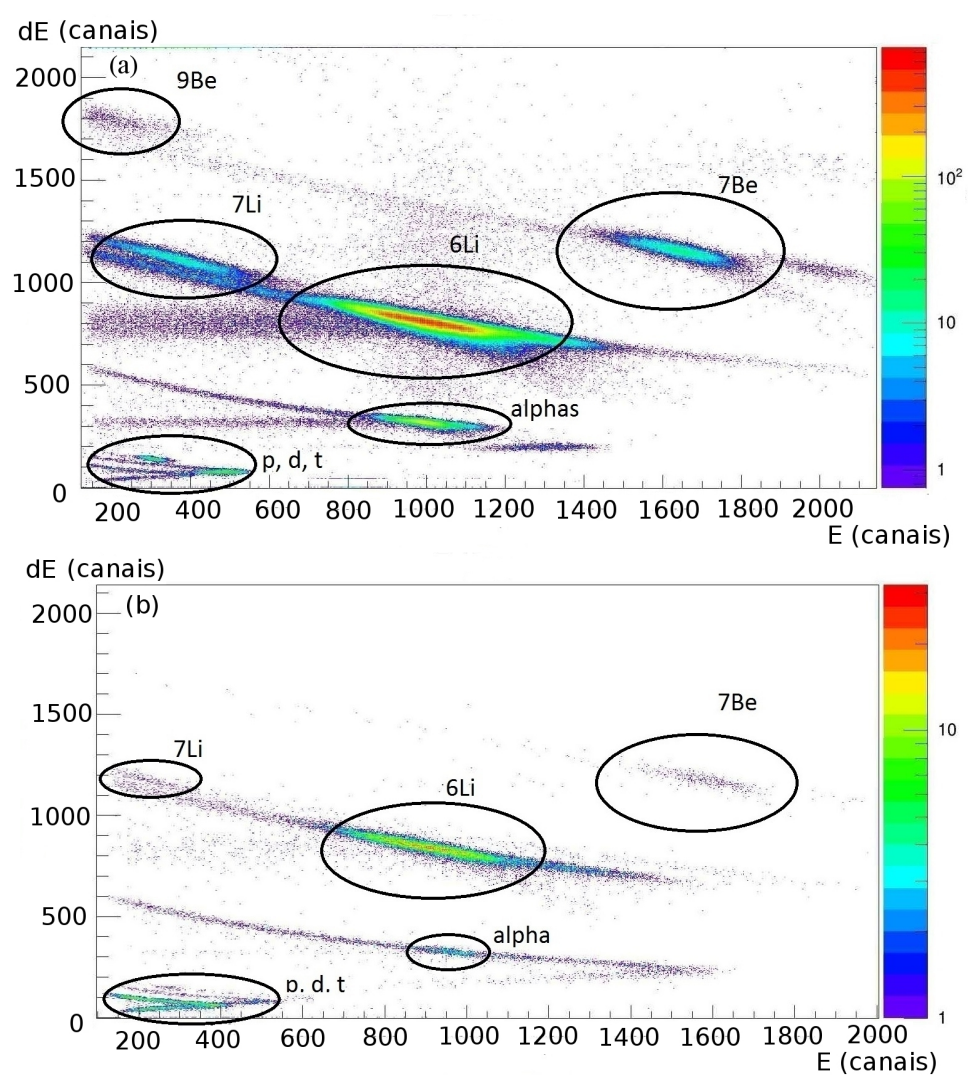

Figura 3.3: Espectros biparamétricos obtidos com feixe de ${ }^{7} \mathrm{Be}$ e alvo de (a) ${ }^{197} \mathrm{Au}$ e (b) ${ }^{9} \mathrm{Be}$ para $\theta_{\text {lab }}=15^{\circ}$. dE é a perda de energia no detector $\Delta \mathrm{E}$ e E é a energia residual.

\subsubsection{Calibração dos Detectores}

A calibração dos detectores é muito importante, pois através dela certifica-se os picos de interesse, além de identificar possíveis reações não inclusas no planejamento. Nesse caso, a calibração foi realizada com o auxílio da fonte alfa $\left({ }^{241} \mathrm{Am}\right)$ e dos picos com melhor definição presentes nos espectros biparamétricos. Foram utilizadas as posições dos picos das partículas (em canais), obtidas através dos espectros, e os valores das perdas de energia correspondentes (em MeV), calculados pelo programa STOPX, para construir retas de calibração $E(M e V)$ x Canal para cada detector. A precisa construção dessas retas nos permite transformar os espectros obtidos em 
canais para energia. A Fig. 3.4 mostra as retas de calibração obtidas. O ajuste linear dessas retas forneceu informações a respeito dos coeficientes a e b, dada a equação da reta: $y=a x+b$. Os resultados podem ser vistos na Tabela 3.2.
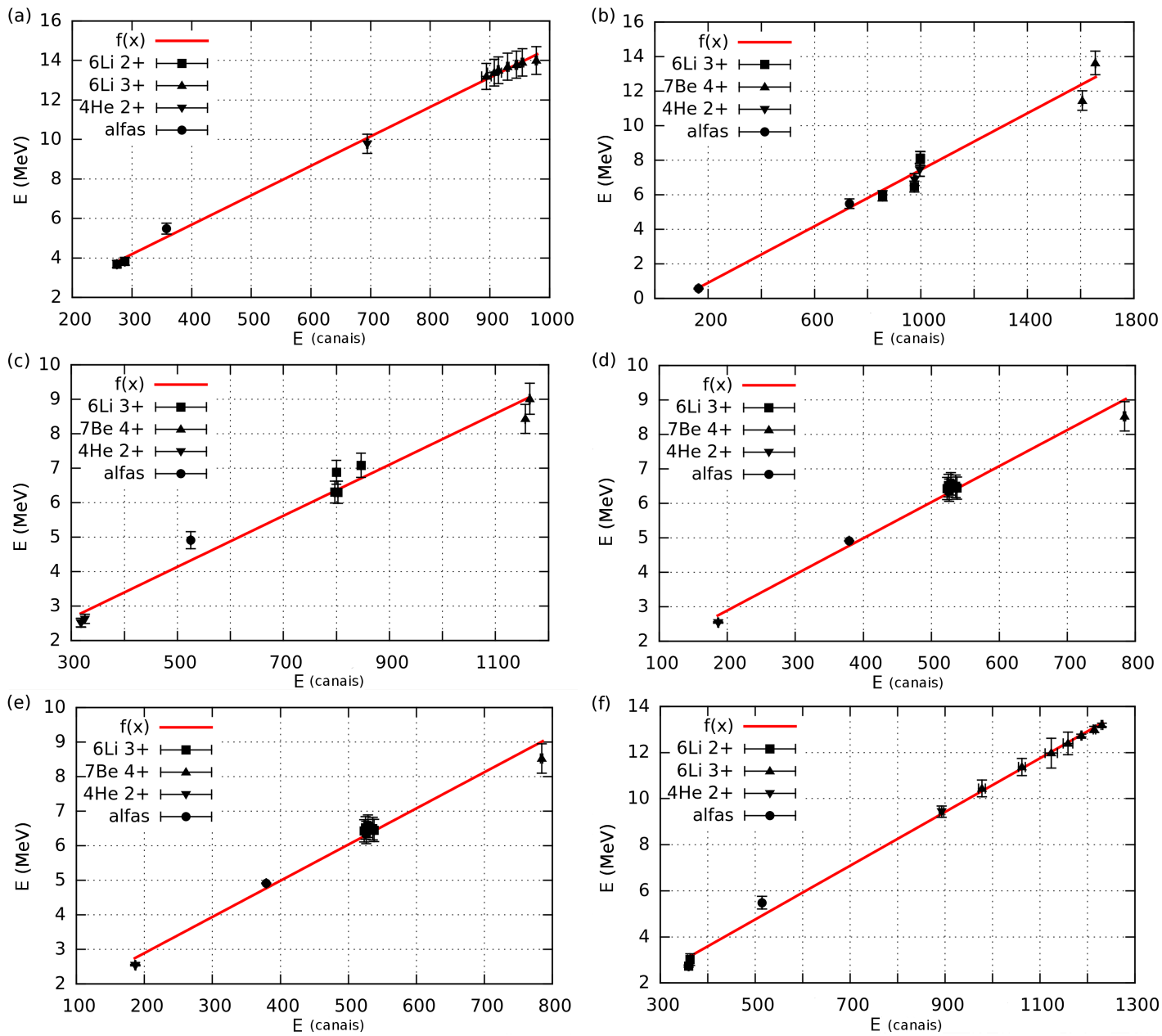

Figura 3.4: Retas calibração para os detectores (a) $E_{1}$ (b) $E_{2}$ (c) $\Delta E_{2}$ (d) $E_{3}$ (e) $\Delta \mathrm{E}_{3}$ (f) $\mathrm{E}_{4}$. Os pontos identificados como alfas representam os dados obtidos pela calibração com a fonte de ${ }^{241} \mathrm{Am}$, enquanto os identificados como $4 \mathrm{He} 2+$ representam as partículas alfas contaminantes obtidas no experimento. Para os detectores $\mathrm{E}_{2}$ e $\mathrm{E}_{3}$, os pontos mostram a calibração realizada com e sem o detector $\Delta \mathrm{E}$ na frente.

Com essas informações foi possível construir espectros biparamétricos $\Delta \mathrm{E} \mathrm{x}$ $\mathrm{E}_{\mathrm{total}^{3}}{ }^{3}$, que fornece a melhor resolução em energia dos picos de interesse. A Fig. 3.5 mostra exemplos dos espectros obtidos.

\footnotetext{
${ }^{3} \mathrm{E}_{\text {total }}$ é a soma das energias perdidas nos detectores $\Delta \mathrm{E}$ e E.
} 
Tabela 3.2: Resultado do ajuste das retas de calibração. As incertezas foram obtidas pelo programa GNUPLOT [28], usado para construir os gráficos deste trabalho.

\begin{tabular}{lcc}
\hline Detector & $\mathrm{a}$ & $\mathrm{b}$ \\
\hline $\mathrm{E} 1$ & $0,0148(2)$ & $-0,2(2)$ \\
$\mathrm{E} 2$ & $0,0081(4)$ & $-0,7(5)$ \\
$\Delta \mathrm{E} 2$ & $0,0074(4)$ & $0,4(3)$ \\
$\mathrm{E} 3$ & $0,0102(2)$ & $-0.5(2)$ \\
$\Delta \mathrm{E} 3$ & $0,0104(4)$ & $0,8(2)$ \\
$\mathrm{E} 4$ & $0,0116(2)$ & $-1,0(2)$ \\
\hline
\end{tabular}
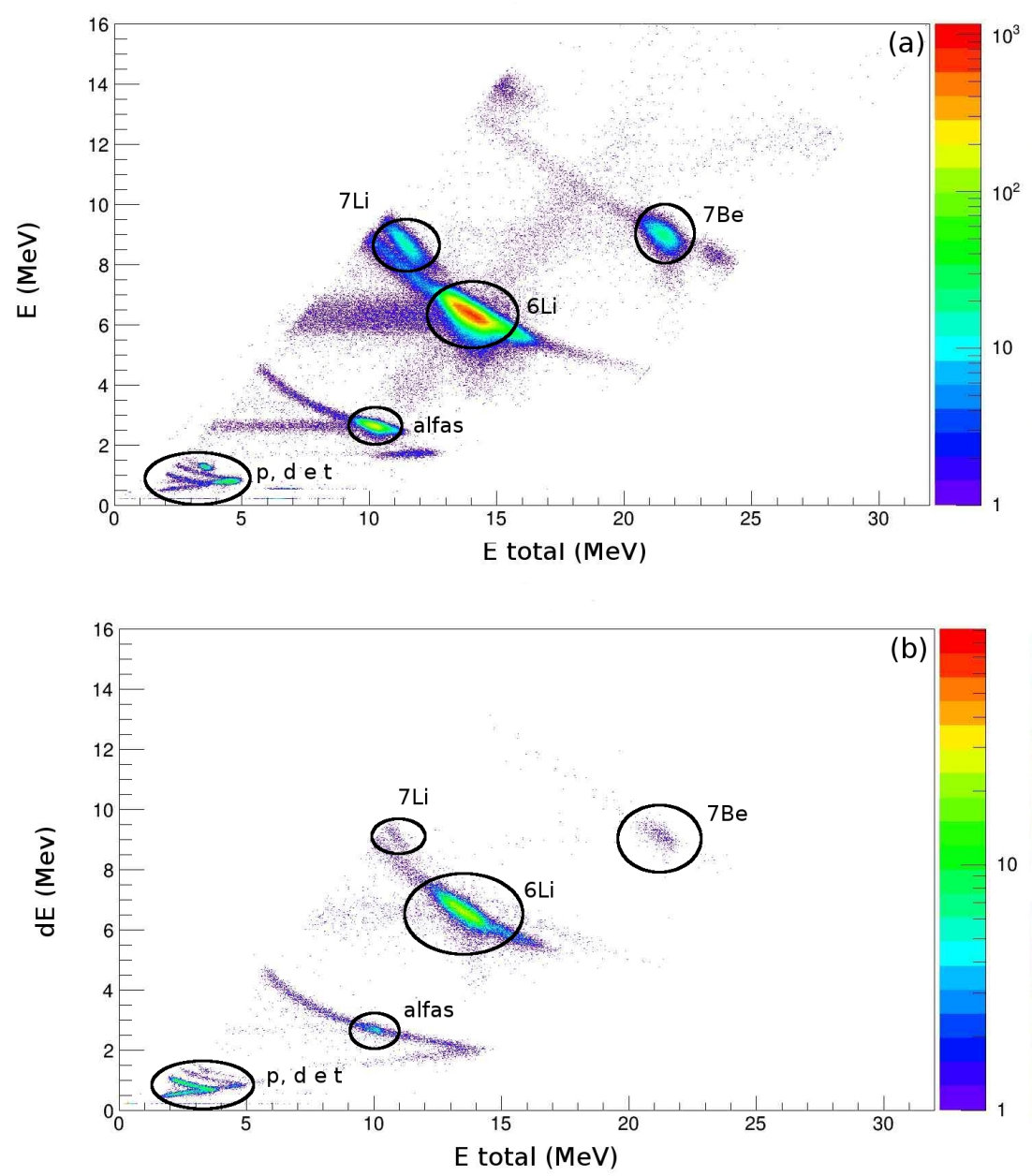

Figura 3.5: Espectros biparamétricos $\Delta \mathrm{E} \times \mathrm{E}_{\text {total }}$ obtidos com feixe de ${ }^{7} \mathrm{Be}$ e alvo de (a) ${ }^{197} \mathrm{Au}$ e (b) ${ }^{9} \mathrm{Be}$ para $\theta_{\text {lab }}=15^{\mathrm{o}}$. 


\subsubsection{Eficiência de Produção}

A normalização dos dados ${ }^{4}$ é realizada através da medida de espalhamento em algum alvo de grande massa, de forma que o potencial de interação entre os núcleos seja Coulombiano, tornando esse espalhamento puramente Rutherford. Neste trabalho foi utilizado um alvo de ${ }^{197} \mathrm{Au}$. A energia da barreira para o sistema ${ }^{7} \mathrm{Be}+{ }^{197} \mathrm{Au}$ é de $\mathrm{E} \approx 45 \mathrm{MeV}$, muito superior comparada com a energia utilizada neste trabalho.

Para o cálculo das seções de choque do sistema ${ }^{7} \mathrm{Be}+{ }^{197} \mathrm{Au}$ normalizadas por Rutherford é necessária a informação da taxa de conversão do feixe primário $\left({ }^{6} \mathrm{Li}\right)$ para o feixe secundário $\left({ }^{7} \mathrm{Be}\right)$, que é obtida utilizando a chamada eficiência $(\epsilon)$, definida como:

$$
\epsilon=\frac{N_{7} B e}{N_{6} L i}
$$

onde $N_{7} B e$ é o número de partículas do feixe secundário e $N_{6} \mathrm{Li}$ é o número de partículas do feixe primário, dado por:

$$
N_{6 i}=\frac{I \times 10^{-10}}{q \times 1,6 \times 10^{-19}}
$$

onde $I$ é o valor da integral de corrente do feixe primário (integrador).

Para o cálculo da quantidade $N_{7}$ Be calcula-se a seção de choque Rutherford para o sistema ${ }^{7} \mathrm{Be}+{ }^{197} \mathrm{Au}$, dada por:

$$
\left(\frac{d \sigma}{d \Omega}\right)_{\text {Ruth }}=\left(\frac{Z_{a} Z_{p}}{4 E_{C M}}\right)^{2} \operatorname{cosc}^{4}\left(\frac{\theta_{C M}}{2}\right)
$$

onde $Z_{a}$ e $Z_{p}$ são os números atômicos do alvo e do projétil, respectivamente, $E_{\mathrm{CM}}$ e $\theta_{\mathrm{CM}}$ são a energia e ângulo de espalhamento no referencial do centro de massa. Sabendo, também, que a seção de choque experimental pode ser calculada a partir do número de contagem dos picos de interesse, através da seguinte equação [10]:

\footnotetext{
${ }^{4}$ Entende-se por normalização dos dados o cálculo da razão $\sigma / \sigma_{R u t h}$, onde pode-se obter a distribuição angular sem interferência do ângulo sólido.
} 


$$
\frac{d \sigma}{d \Omega}=\frac{N_{d} J}{N_{a} N_{f} \Delta \Omega}
$$

onde $N_{d}$ é o número de contagens no pico de interesse, $J$ é o fator de conversão do sistema do laboratório para o sistema do centro de massa (Jacobiano), $N_{a}$ é o número de átomos $/ \mathrm{cm}^{2}$ no alvo secundário e $N_{f}$ é o número de partículas do feixe secundário. Assim, é possível escrever ${ }^{5}$ :

$$
N_{{ }^{B} \mathrm{Be}}=\frac{N_{7_{B e}^{197} A u} J}{\sigma_{7_{B e+}^{R u t h}}^{\text {Rer } A u}{ }^{N_{197} A u} \Delta \Omega}
$$

Resolvendo a Eq. (3.3) utilizando as Eqs. (3.4) e (3.7), foi possível notar uma pequena variação na eficiência de produção ao longo do experimento, indicando uma possível não homogeneidade ou degradação do alvo primário durante o experimento. O gráfico da eficiência de produção no decorrer do experimento pode ser visto na Fig. 3.6.

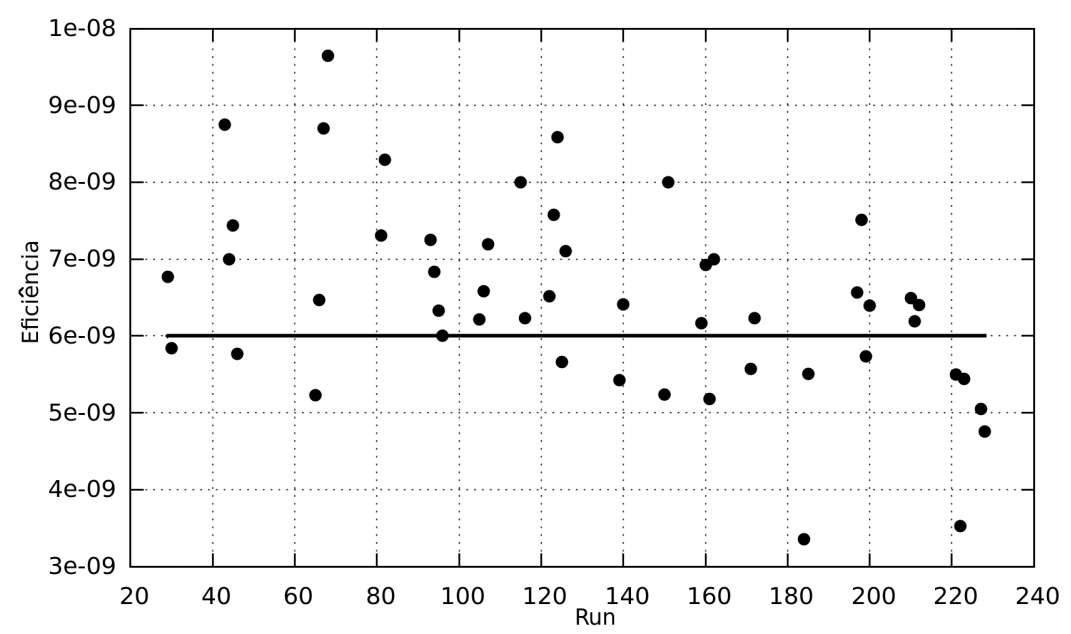

Figura 3.6: Variação da eficiência de produção ao longo do experimento. Mostrando a média $\left(\bar{\epsilon} \approx 6 \times 10^{-9}\right)$ obtida ao longo do experimento.

\footnotetext{
${ }^{5}$ Onde $\frac{d \sigma}{d \Omega}$ foi denotado por $\sigma$ para simplificar a notação.
} 


\subsection{Distribuições Angulares}

Distribuições angulares envolvendo feixes exóticos são geralmente normalizadas utilizando-se informações do espalhamento do mesmo projétil em um alvo de ${ }^{197} \mathrm{Au}$, visto que não tem-se uma medida direta da intensidade do feixe secundário.

\subsubsection{Correção Angular}

Fatores ligados à geometria do experimento podem mudar sensivelmente os valores obtidos para a seção de choque experimental, principalmente em ângulos dianteiros. Em espalhamentos com feixes de baixa intensidade geralmente utiliza-se detectores com ângulo sólido grande para que mais partículas sejam detectadas em cada medida, isso produz uma aceitação angular grande, fazendo com que detectores posicionados em um ângulo $\theta$ detectem partículas com ângulo de espalhamento entre $\theta \pm \Delta \theta$.

O sistema RIBRAS produz feixes com certa divergência angular $\left(1,5^{\circ}-4,5^{\circ}\right)$, gerada pelo ângulo de aceitação dos solenoides e pelo fato de o feixe sofrer straggling ${ }^{6}$ angular e em energia quando atravessa o alvo secundário. Diante disso, torna-se necessário uma correção nos ângulos medidos experimentalmente, que foi realizada utilizando o código RIBRAS I [29], que usa o Método de Monte Carlo para simular o espalhamento dos feixes e calcula um ângulo médio de espalhamento das partículas. Essa correção torna-se mais importante em ângulos dianteiros, onde a probabilidade de as partículas serem espalhadas é maior, como é previsto pela Eq. (3.5). A Fig. 3.7 mostra a distribuição angular ${ }^{7} \mathrm{Be}+{ }^{197} \mathrm{Au}$ antes e depois da correção angular.

\subsubsection{Distribuições Angulares Elásticas}

Escrevendo a Eq. (3.6) para as medidas dos espalhamentos ${ }^{9} \mathrm{Be}\left({ }^{7} \mathrm{Be},{ }^{7} \mathrm{Be}\right)$ e ${ }^{197} \mathrm{Au}\left({ }^{7} \mathrm{Be},{ }^{7} \mathrm{Be}\right)$, é possível calcular a razão:

\footnotetext{
${ }^{6}$ Variação no ângulo de espalhamento e perdas de energia devido aos diferentes percursos e interações da partícula com o alvo
} 


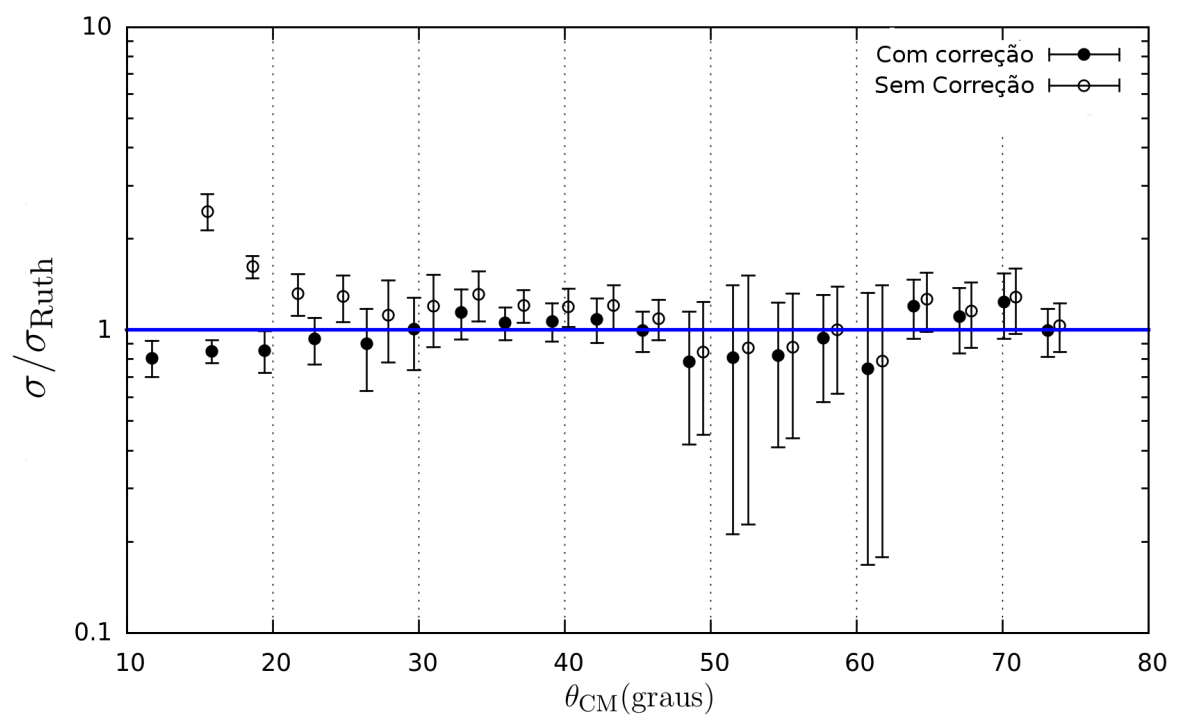

Figura 3.7: Distribuição angular ${ }^{7} \mathrm{Be}+{ }^{197} \mathrm{Au}$ para $\mathrm{E}_{\mathrm{lab}}=23,3 \mathrm{MeV}$, com e sem a correção angular utilizando o código RIBRAS I.

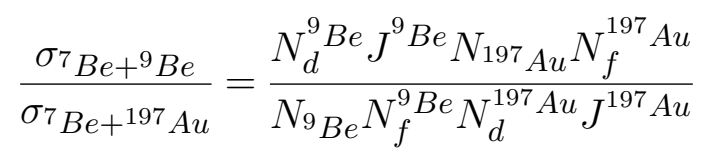

onde o sobrescrito se refere ao alvo utilizado e $\frac{d \sigma}{d \Omega}$ foi denotado por $\sigma$ para simplificar a notação.

Como não tem-se medidas diretas da intensidade do feixe secundário, faz-se necessário a seguinte consideração:

$$
\frac{N_{f}^{197} A u}{N_{f}^{9} B e}=\frac{\epsilon}{\epsilon} \frac{I^{197} A u}{I^{9} B e}=\frac{I^{197} A u}{I^{9} B e}
$$

onde $I$ é o integrador. Substituindo a Eq. (3.9) na Eq. (3.8) e dividindo ambos os lados por $\sigma_{7 B e+{ }^{9} B e}^{R u t h}$, obtém-se a seção de choque experimental normalizada, relativa à Rutherford ${ }^{7}$ :

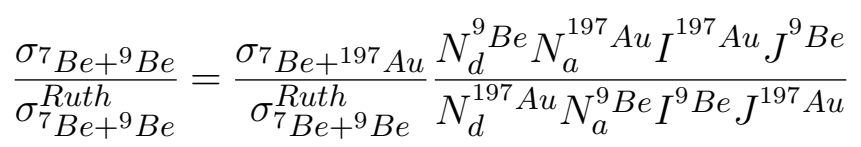

\footnotetext{
${ }^{7}$ Como foi visto na Eq. 3.9, não há mais dependência com a eficiência, eliminando os erros referentes a variação de $\epsilon$.
} 
onde $\sigma_{7 B e+{ }^{9} B e}^{R u t h}$ é dada pela Eq. (3.5). A Fig. 3.8 apresenta a distribuição angular normalizada e absoluta obtida para o sistema ${ }^{7} \mathrm{Be}+{ }^{9} \mathrm{Be}$ a $\mathrm{E}_{\text {lab }}=23,1 \mathrm{MeV}$.

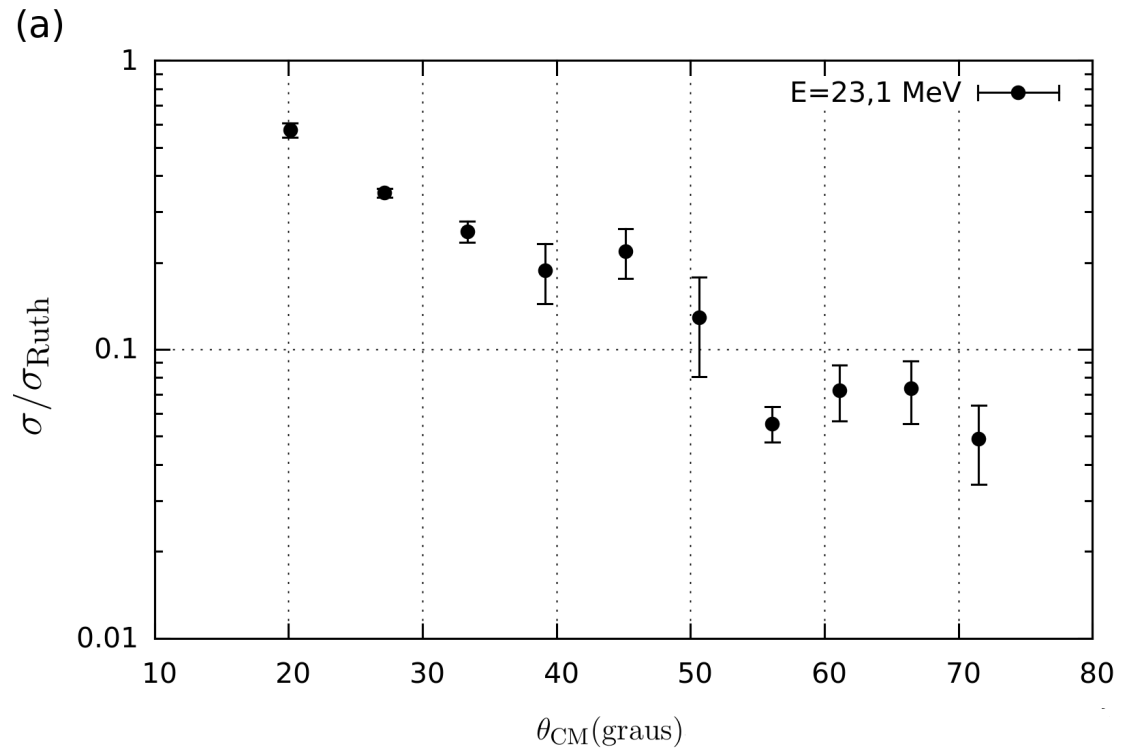

(b)

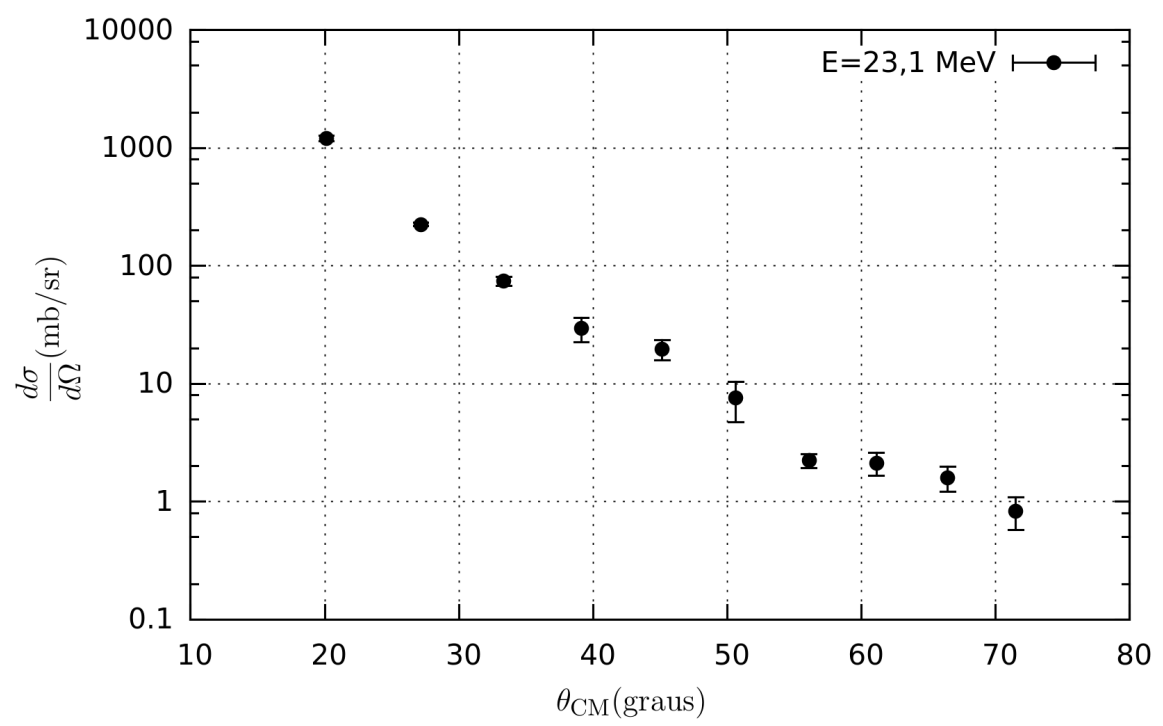

Figura 3.8: Distribuição angular normalizada (a) e absoluta (b) para o espalhamento quasielástico ${ }^{7} \mathrm{Be}+{ }^{9} \mathrm{Be}$ a $\mathrm{E}_{\mathrm{lab}}=23,1 \mathrm{MeV}$.

Observando a Fig. 3.8 pode-se notar um comportamento oscilatório da seção de choque em função do ângulo de espalhamento. É importante mencionar que os valores dos ângulos de espalhamento já levam em consideração a correção angular realizada utilizando o programa RIBRAS I, mencionada anteriormente. 


\subsubsection{Comparação com dados da literatura}

Os dados obtidos neste trabalho foram comparados com medidas já realizadas anteriormente para o espalhamento ${ }^{7} \mathrm{Be}+{ }^{9} \mathrm{Be}$ a $\mathrm{E}_{\mathrm{lab}}=23,7 \mathrm{MeV}$ por $I$. Mukha et $a l^{8}[2]$ (ver Fig. 3.9(a)) e a $\mathrm{E}_{\mathrm{lab}}=21 \mathrm{MeV}$ por S. Verma et al ${ }^{9}$ [1] (ver Fig. 3.9(b)).

(a)

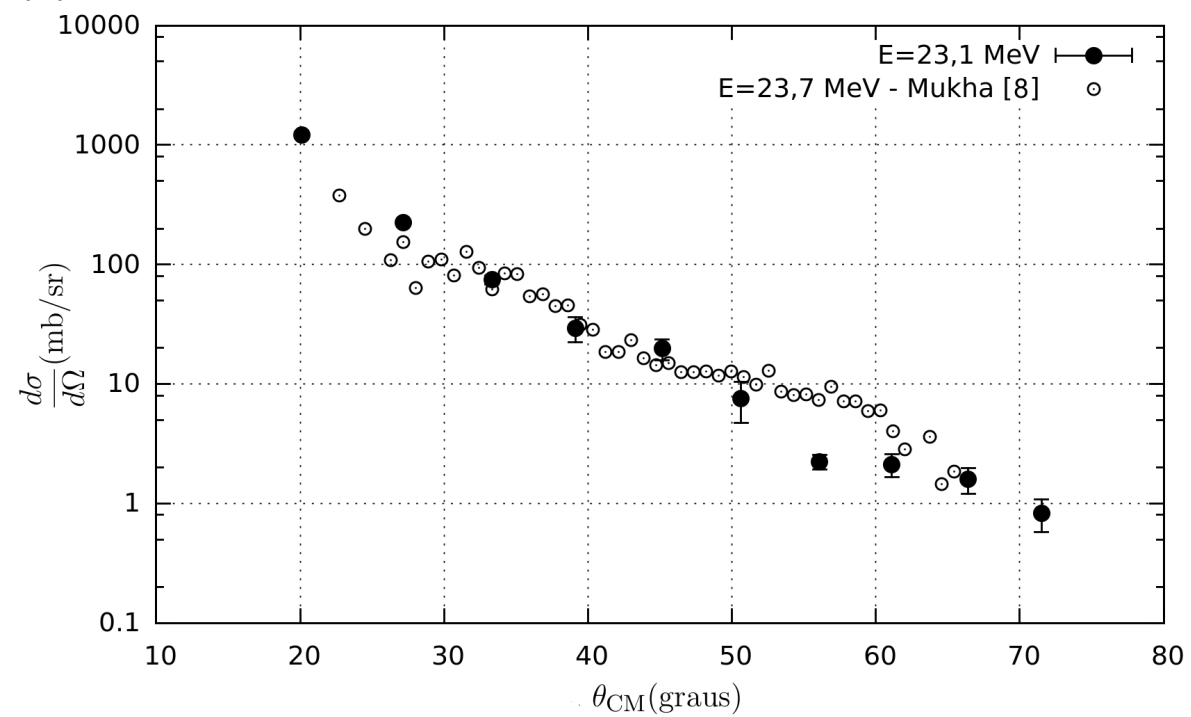

(b)

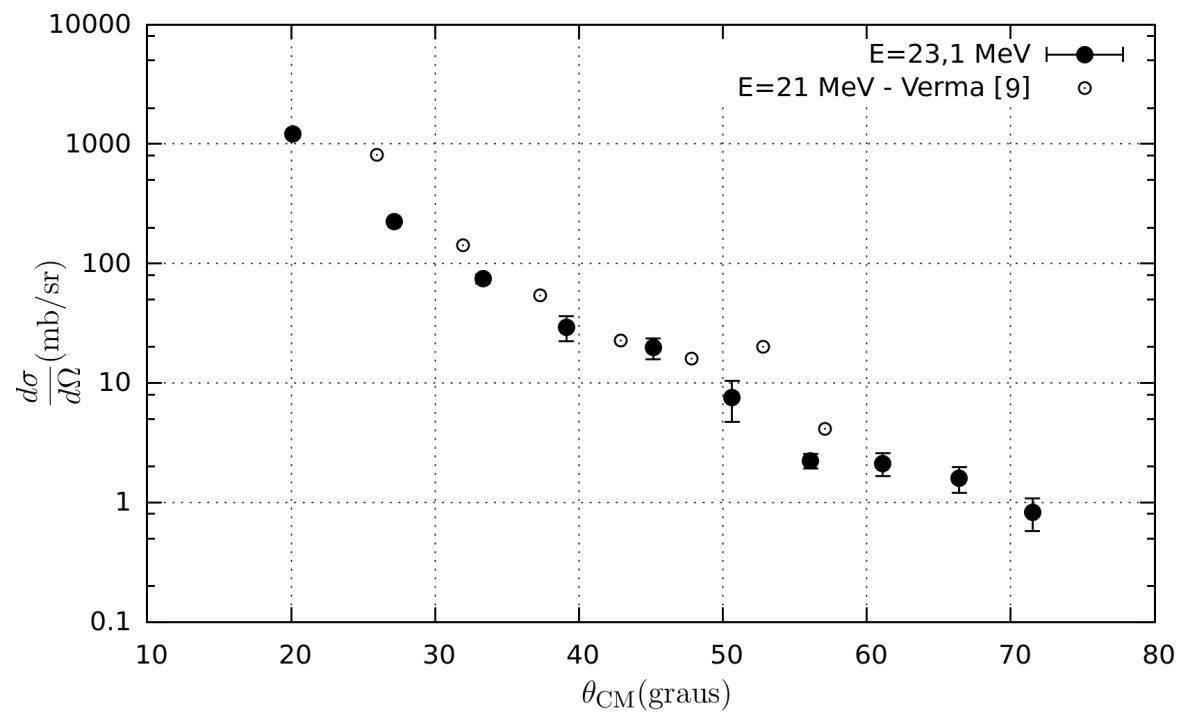

Figura 3.9: Comparação com dados obtidos por (a) Mukha et al [2] e (b) S. Verma et al [1], com as respectivas energias.

Observando a Fig. 3.9(a) nota-se uma pequena discrepância entre os dados na

${ }^{8}$ Pesquisador do Instituto Helmholzzentrum für Schwerionenforschung, Alemanha;

${ }^{9}$ Pesquisadora do Department of Physics and Astrophysics da University of Delhi. 
região de $\theta_{\mathrm{CM}} \approx 55^{\circ}$. No caso da Fig. $3.9(\mathrm{~b})$ observa-se uma ligeira diferença nos ângulos dianteiros.

As discrepâncias observadas podem ser devido às diferenças intrínsecas aos dois sistemas, como, por exemplo, a diferença de energia e a resolução angular ou até mesmo a aceitação angular dos sistemas utilizados para aquisição dos dados. Entretanto, as medidas são compatíveis entre si e mostram que os dados obtidos neste trabalho estão consistentes com dados encontrados na literatura.

\subsubsection{Transferência Elástica}

Nos espectros biparamétricos obtidos na aquisição de dados do espalhamento quasielástico ${ }^{9} \mathrm{Be}\left({ }^{7} \mathrm{Be},{ }^{7} \mathrm{Be}\right){ }^{9} \mathrm{Be}$ foram observadas contagens acima do pico de ${ }^{7} \mathrm{Be}$, como pode ser visto na Fig. 3.10.

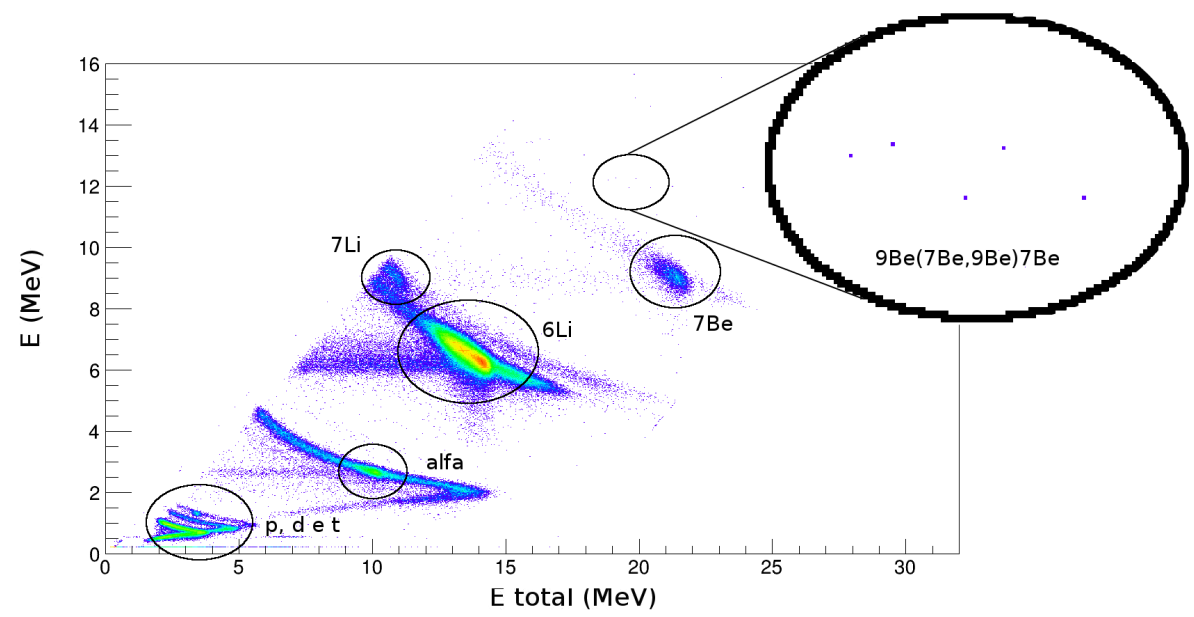

Figura 3.10: Espectro biparamétrico $\Delta \mathrm{E} \times \mathrm{E}_{\text {Total }}$ obtido com feixe de ${ }^{7} \mathrm{Be}$ e alvo de ${ }^{9}$ Be para $\theta_{\mathrm{CM}}=15^{\circ}$. É possível observar as contagens referentes ao processo de transferência elástica ${ }^{9} \mathrm{Be}\left({ }^{7} \mathrm{Be},{ }^{9} \mathrm{Be}\right){ }^{7} \mathrm{Be}$.

Através das retas de calibração foi possível verificar que esses dados referem-se à transferência elástica ${ }^{9} \mathrm{Be}\left({ }^{7} \mathrm{Be},{ }^{9} \mathrm{Be}\right){ }^{7} \mathrm{Be}$, onde há uma transferência de 2 nêutrons do ${ }^{9}$ Be para o ${ }^{7}$ Be durante a colisão. Como os canais de entrada e saída são iguais nos dois casos, a medida da transferência elástica pode ser utilizada para complementar a distribuição angular do espalhamento quasielástico ${ }^{7} \mathrm{Be}+{ }^{9} \mathrm{Be}$ na região de ângulos traseiros, já que a detecção de um ${ }^{9} \mathrm{Be}$ em ângulos dianteiros corresponde a um ${ }^{7} \mathrm{Be}$ 
em ângulos traseiros. Porém, a contribuição da transferência elástica não pode ser separada da parte puramente elástica da seção de choque, fazendo com que a análise tenha que ser feita separadamente.

O ângulo de recuo do ${ }^{7} \mathrm{Be}$ pode ser obtido pelas leis de conservação de momento e energia que fornecem a relação $\theta_{C M}^{7} B e=\pi-\theta_{C M}^{9} B e$, onde $\theta_{C M}^{9} B e$ é o ângulo de espalhamento do ${ }^{9} \mathrm{Be}$ em ângulos dianteiros no referencial do centro de massa.

A partir das contagens obtidas foi possível estimar a seção de choque para esse processo. A distribuição angular foi calculada e os resultados obtidos podem ser vistos na Fig. 3.11. Devido ao fato de o número de contagens ser extremamente baixo as incertezas obtidas foram relativamente grandes.

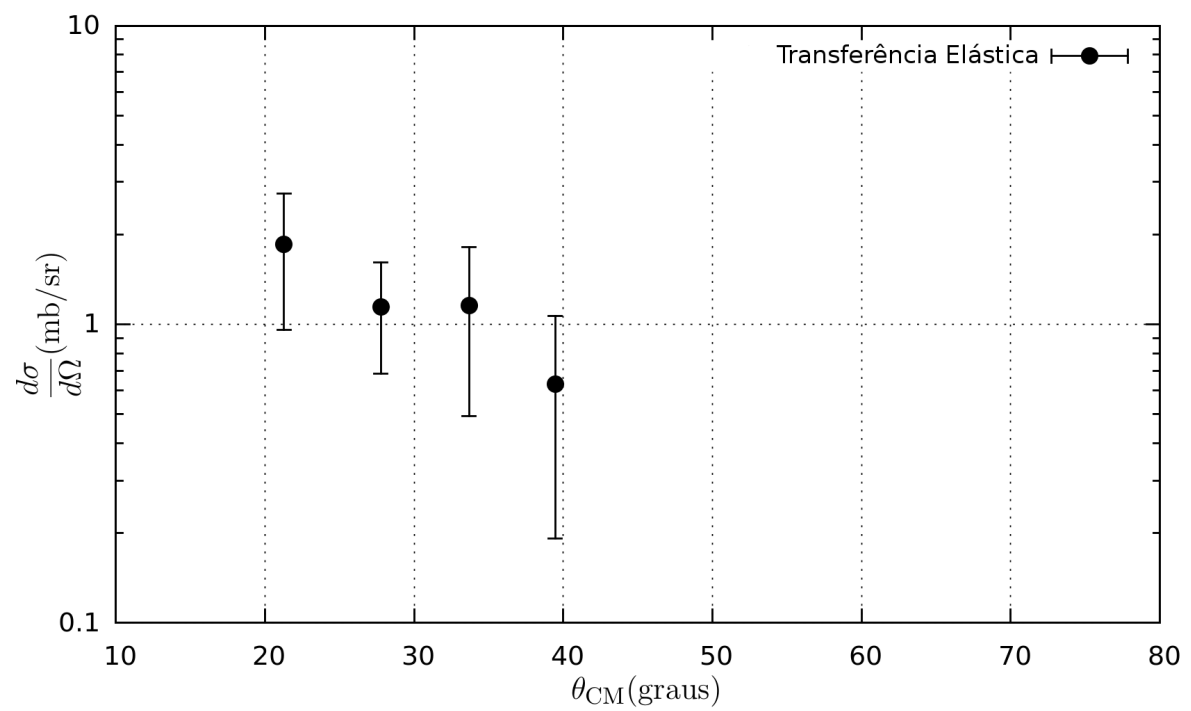

Figura 3.11: Distribuição angular da reação de transferência ${ }^{9} \mathrm{Be}\left({ }^{7} \mathrm{Be},{ }^{9} \mathrm{Be}\right){ }^{7} \mathrm{Be}$ somada à detecção de ${ }^{9} \mathrm{Be}$ de recuo em ângulos dianteiros.

Esses dados puderam, então, ser incluídos na distribuição angular para o espalhamento ${ }^{7} \mathrm{Be}+{ }^{9} \mathrm{Be}$ já apresentada anteriormente (ver Fig. 3.8(b)). A Fig. 3.12 apresenta a distribuição angular completa. 


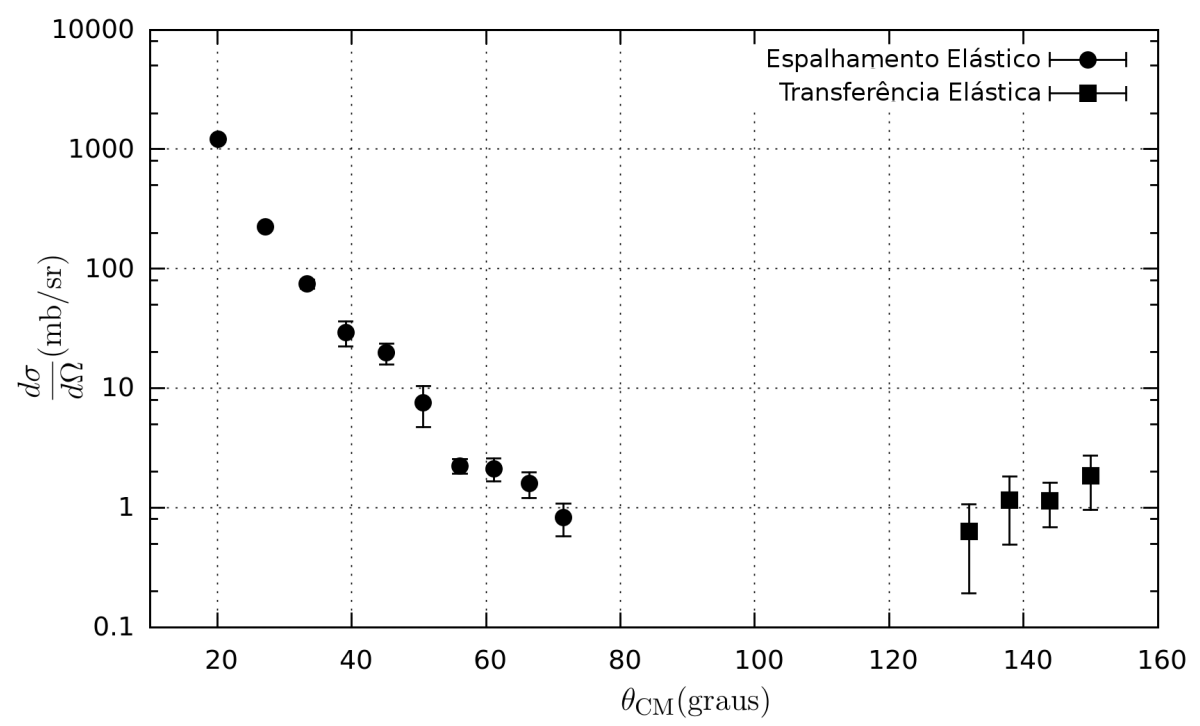

Figura 3.12: Distribuição angular do espalhamento quasielástico ${ }^{7} \mathrm{Be}+{ }^{9} \mathrm{Be}$ somada à contribuição referente à transferência elástica em ângulos traseiros. 


\section{Capítulo 4}

\section{Fundamentos Teóricos}

Nesse Capítulo será feita uma breve descrição sobre as reações diretas, espalhamento Rutherford e espalhamento para potenciais nucleares de curto alcance. Além disso, será apresentada a descrição teórica necessária para a análise do espalhamento ${ }^{7} \mathrm{Be}+{ }^{9} \mathrm{Be}$, bem como a influência do primeiro estado excitado do núcleo ${ }^{7} \mathrm{Be}$, que não pôde ser separado devido à baixa resolução experimental. A análise foi feita através dos formalismos de Modelo Óptico, Canais Acoplados e Aproximação de Born de Ondas Distorcidas.

\subsection{Reações Diretas}

O conceito de uma interação nuclear forte de curto alcance induz o conceito de que reações nucleares se processam unicamente pela formação de um núcleo composto, onde a energia incidente seria aleatoriamente distribuída entre os núcleons. Esse conceito ignora completamente os casos onde a interação nuclear é realizada próxima à borda de alcance da força nuclear forte. Nessa região, onde a interação nuclear de curto alcance sobrepuja a Coulombiana, existe uma grande probabilidade de que a partícula incidente seja rapidamente espalhada. Essas reações em que a interação núcleo-alvo acontece em tempos da ordem de $10^{-22}$ s e há um rearranjo mínimo na estrutura nuclear dos constituintes são chamadas de reações diretas, como por exemplo os espalhamentos elásticos, inelásticos, reações de transferências como pick-up ou stripping, ou então o processo de knock-out. A Fig. 4.1 mostra um 
esquema das reações diretas e com formação de núcleo composto.

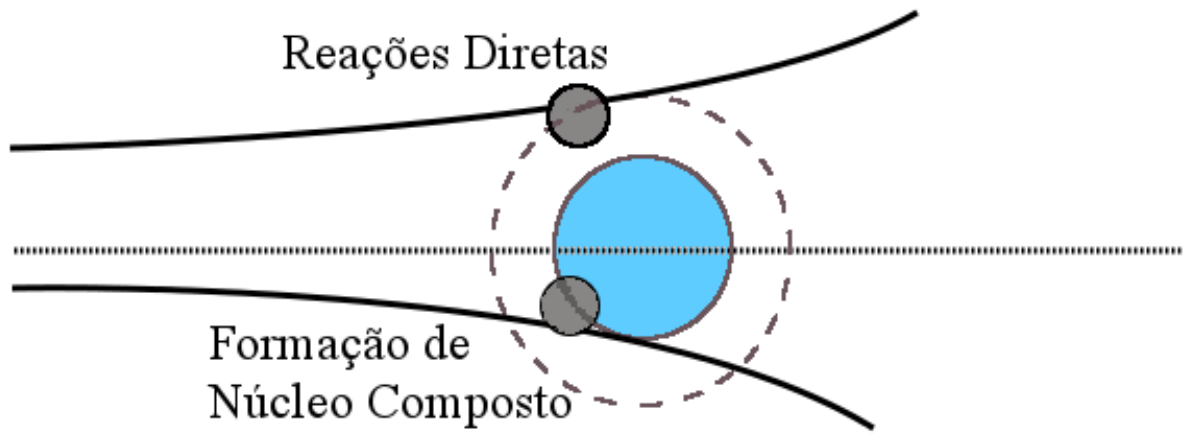

Figura 4.1: Espalhamento através de reações diretas e pela formação de núcleo composto.

\subsection{Espalhamento Elástico}

Diversos processos podem ocorrer durante uma colisão nuclear, como o espalhamento elástico, o espalhamento inelástico, a transferência de núcleons, etc. Dentre todos os processos, o mais comum de ocorrer é o espalhamento elástico, onde as partículas incidente e espalhada têm a mesma estrutura, não havendo transferência de núcleons nem excitação dos núcleos espalhados, podendo haver apenas mudança na orientação do spin. Pode-se, portanto, descrever a partícula espalhada com os mesmos números quânticos da partícula incidente.

Potenciais centrais são extremamente comuns na teoria de espalhamento, pois as interações entre núcleons são representadas pelas forças Coulombianas e Nucleares, ambas centrais. Portanto, pode-se escrever a equação de Schrödinger como:

$$
\left[-\nabla^{2}+\frac{2 \mu}{\hbar^{2}} V(r)\right] \Psi(\vec{r})=k^{2} \Psi(\vec{r})
$$

onde $k^{2}=\frac{2 \mu E}{\hbar^{2}}$ e $\mu=\frac{m_{p} m_{a}}{m_{p}+m_{a}}$ é a massa reduzida do sistema. Para os potenciais centrais tratados nesse trabalho, teremos: 


$$
V(r)=V_{N}(r)+V_{C}(r)
$$

onde $V_{N}(r)$ representa o potencial nuclear e $V_{C}(r)$ o potencial Coulombiano. Desta forma, a equação de Schrödinger pode ser resolvida pelo método de separação de variáveis:

$$
\Psi=\sum_{l} a_{l} \frac{u_{l}(r)}{k r} P_{l}(\cos \theta)
$$

onde $P_{l}(\cos \theta)$ são os polinômios de Legendre, soluções da parte angular da Eq. (4.1), e $u_{l}(r)$ é solução da parte radial:

$$
\frac{d^{2} u(r)}{d r^{2}}+\frac{2 m}{\hbar^{2}}\left[E-V(r)-\frac{\hbar^{2}}{2 m} \frac{l(l+1)}{r^{2}}\right] u(r)=0
$$

A distância dos centros espalhadores aos detectores é muito maior que as dimensões nucleares, de tal forma que será de interesse o uso da parte assintótica da função de onda radial espalhada. A partícula incidente pode ser descrita como uma onda plana, que ao ser espalhada por um potencial central assume a forma de uma onda esférica emergente. Com isso, a função de onda assintótica será a soma da onda incidente com a onda espalhada:

$$
\Psi \stackrel{r \rightarrow \infty}{\rightarrow} e^{i k z}+f(\theta) \frac{e^{i k r}}{r}
$$

onde $f(\theta)$ é a amplitude de espalhamento nuclear, responsável por mostrar que as direções de espalhamento não são igualmente prováveis nessa coordenada.

A seção de choque diferencial e a amplitude de espalhamento podem ser associadas pela expressão:

$$
\frac{d \sigma}{d \Omega}=|f(\theta)|^{2}
$$




\subsubsection{Espalhamento Rutherford}

Para o espalhamento em um potencial central fora do alcance da força nuclear, pode-se escrever o termo relacionado ao potencial Coulombiano ${ }^{1}$ da seguinte forma $[30]:$

$$
V_{C}(r)=\left\{\begin{array}{cc}
\frac{Z_{a} Z_{p} e^{2}}{2 R_{C}}\left(3-\frac{r^{2}}{R_{C}^{2}}\right) & \left(r \leq R_{C}\right) \\
\frac{Z_{a} Z_{p} e^{2}}{r} & \left(r>R_{C}\right)
\end{array}\right.
$$

onde $R_{C}$ é o raio da barreira Coulombiana, $Z_{a}$ é o número de prótons do alvo, $Z_{p}$ é o número de prótons do projétil e e a carga elementar. Assim a Eq. 4.1 toma a forma [30]:

$$
\left[-\nabla^{2}+\frac{2 \eta k}{r}\right] \Psi(\vec{r})=k^{2} \Psi(\vec{r})
$$

onde $\eta=\frac{Z_{a} Z_{p} e^{2}}{\hbar v}$ é conhecido como o fator de Sommerfeld.

Considerando apenas a contribuição devido ao potencial Coulombiano, a solução da equação de Schrödinger é dada por:

$$
\Psi_{C}(\vec{r})=\Phi_{i}(\vec{r})+\Phi_{e}(\vec{r})
$$

ou seja, a soma das funções de onda incidente $\left(\Phi_{i}(\vec{r})\right)$ e espalhada $\left(\Phi_{e}(\vec{r})\right)$.

Apenas a parte espalhada na zona assintótica é importante no caso tratado, desta forma:

$$
\Psi \stackrel{r \rightarrow \infty}{\rightarrow} f_{C}(\theta) \frac{e^{i[k r-\eta \ln (2 k r)]}}{r}
$$

com a amplitude de espalhamento Rutherford dada por:

$$
f_{C}(\theta)=-\frac{\eta}{2 k \operatorname{sen}^{2}\left(\frac{\theta}{2}\right)} e^{i\left\{\eta \ln \left[\operatorname{sen}^{2}\left(\frac{\theta}{2}\right)\right]+2 \sigma_{0}\right\}}
$$

\footnotetext{
${ }^{1}$ No contexto de Física Nuclear, potencial Coulombiano refere-se à energia potencial elétrica.
} 
onde $\sigma_{0}$ é a defasagem correspondente ao potencial Coulombiano. Substituindo a expressão acima na Eq. (4.6) [30]:

$$
\frac{d \sigma}{d \Omega}=\frac{\eta^{2}}{4 k^{2} \operatorname{sen}^{4}\left(\frac{\theta}{2}\right)}=\left(\frac{Z_{a} Z_{p}}{4 E_{C M}}\right)^{2} \operatorname{cosc}^{4}\left(\frac{\theta_{C M}}{2}\right)
$$

\subsubsection{Espalhamento por um potencial adicional de curto al- cance}

Em um espalhamento geral, juntamente com o potencial Coulombiano tem-se o potencial nuclear. Desta forma, o potencial total é da forma apresentada na Eq. (4.2), ou seja, a soma de ambas as contribuições. Com isso, a equação de Schrödinger pode ser escrita como [30]:

$$
\left[-\nabla^{2}+\frac{2 \eta k}{r}+\frac{2 \mu}{\hbar^{2}} V_{N}(r)\right] \Psi(\vec{r})=k^{2} \Psi(\vec{r})
$$

que tem solução assintótica na forma:

$$
\Psi \stackrel{r \rightarrow \infty}{\rightarrow} f_{N}(\theta) \frac{e^{i[k r-\eta \ln (2 k r)]}}{r}
$$

Assim, forma a amplitude de espalhamento é escrita como:

$$
f_{N}(\theta)=\frac{1}{2 i k} \sum_{l}(2 l+1) e^{2 i \sigma_{l}}\left(S_{l}-1\right) P_{l}(\cos \theta)
$$

onde $S_{l}$ corresponde aos elementos diagonais da matriz de espalhamento elástico ou matriz $\mathrm{S}$, e $P_{l}$ são os polinômios de Legendre, que carregam a dependência angular das soluções da equação de Schrödinger.

Com isso, a amplitude de espalhamento total será dada pela soma das componentes Coulombiana e Nuclear:

$$
f(\theta)=f_{C}(\theta)+f_{N}(\theta)
$$


assim:

$$
\frac{d \sigma}{d \Omega}=|f(\theta)|^{2}=\left|f_{C}(\theta)+f_{N}(\theta)\right|^{2}=\left|f_{C}(\theta)\right|^{2}+2 R e\left(f_{C}^{*} f_{N}\right)+\left|f_{N}(\theta)\right|^{2}
$$

\subsubsection{Modelo Óptico (MO)}

O modelo óptico foi introduzido com o intuito de resolver algumas inconsistências que existiam com as expressões analíticas obtidas a partir da suposição de que a partícula incidente era sempre absorvida quando atingisse o núcleo alvo. Como exemplo, podemos citar:

- indicativo de que a seção de choque deveria decrescer com a energia e crescer $\operatorname{com} A^{1 / 3}$

- falta de explicação com relação ao comportamento ondulatório da seção de choque total para o espalhamento de nêutrons.

Em 1953, H. Feshbach et al [31] introduziram as bases desse modelo partindo do conceito de que o núcleo alvo tem certa chance de não absorver a partícula incidente, ou seja, a probabilidade de a partícula ser absorvida é menor que 1. Esse modelo foi inspirado no espalhamento da luz por um meio com índice de refração complexo, onde o potencial mais geral é dado pela soma dos termos:

$$
U_{N}(r)=U_{R}(r)+U_{I}(r)+U_{D}(r)+U_{S}(r)
$$

o significado de cada termo será discutido na próxima subseção. Esse modelo tem boa precisão em explicar as oscilações existentes em distribuições elásticas devido a estados excitados no sistema composto $^{1}$ e por estados residuais nos canais não elásticos [32].

\footnotetext{
${ }^{1}$ Sistema de interação máxima entre o núcleo alvo e a partícula incidente.
} 


\subsubsection{Potenciais Ópticos}

Os potenciais apresentados na Eq. (4.18) representam as possíveis interações que podem ocorrer no momento de uma colisão. O primeiro termo, $U_{R}(r)$, representa a parte real do potencial nuclear [33]:

$$
U_{R}(r)=-V f(r, R, a)
$$

onde $V$ é profundidade do poço, $R$ é o raio nuclear, $a$ é a difusividade superficial e $f(r, R, a)$ é o fator de forma, que pode, por exemplo, ser descrito pelos potenciais de Woods-Saxon ou de São Paulo.

Os efeitos de absorção são descritos por duas parcelas do potencial óptico [33]:

$$
\begin{gathered}
U_{I}(r)=-i W_{I} f\left(r, R_{i}, a_{i}\right) \\
U_{D}(r)=4 i W_{D} \frac{d}{d r} f\left(r, R_{i}, a_{i}\right)
\end{gathered}
$$

onde $U_{I}(r)$ e $U_{D}(r)$ são as partes imaginárias de volume e superfície do potencial, respectivamente, e representam a remoção do fluxo incidente do canal de entrada (canal elástico) para outros canais de reação. Os termos $W_{I}$ e $W_{D}$ são as profundidades dos potenciais e atuam de forma complementar: $W_{D}$ é predominante em baixas energias, onde as interações ocorrem basicamente na superfície e $W_{I}$ domina em altas energias, onde a partícula incidente atinge maior profundidade.

A interação spin-órbita é descrita pela equação [33]:

$$
U_{S}(r)=\vec{s} \cdot \vec{l}\left(\frac{\hbar}{M_{\pi} c^{2}}\right)^{2} V_{s o} \frac{1}{r} \frac{d}{d r} f\left(r, R_{s o}, a_{s o}\right)
$$

onde $\vec{s}$ e $\vec{l}$ são os operadores de spin e momento angular respectivamente, $M_{\pi}$ é a massa do píon. Essa parcela é importante para descrever o efeito de polarização e é extremamente pequena, sendo geralmente ignorada nos cálculos experimentais. 


\subsubsection{Potencial Woods-Saxon}

Uma das parametrizações mais utilizadas na análise de dados experimentais é a proposta por Woods e Saxon em 1954 [34]. Essa parametrização foi capaz de obter soluções exatas para as equações de Schrödinger e Klein-Gordon para um nêutron se movendo em um potencial central. Esse fator de forma é escrito como:

$$
f(r, R, a)=\frac{1}{1+e^{(r-R) / a}}
$$

onde $r, R$ e $a$ já foram definidos anteriormente. Levando em consideração a incompressibilidade do núcleo atômico pode-se associar o raio nuclear $(\mathrm{R})$ com o número de núcleons $(A)$, do projétil $\left(A_{P}\right)$ e alvo $\left(A_{T}\right)$, e com o raio reduzido $\left(r_{0}\right)$ através da equação:

$$
R=r_{0}\left(A_{T}^{1 / 3}+A_{P}^{1 / 3}\right)
$$

A forma do potencial de Woods-Saxon, apresentado na Fig. 4.2, tem as bordas mais arredondadas que um potencial do tipo poço quadrado, sendo assim, mais realista que os potenciais de curto alcance comuns e vai rapidamente a zero quando a distância entre os núcleos ultrapassa a distância de interação nuclear.

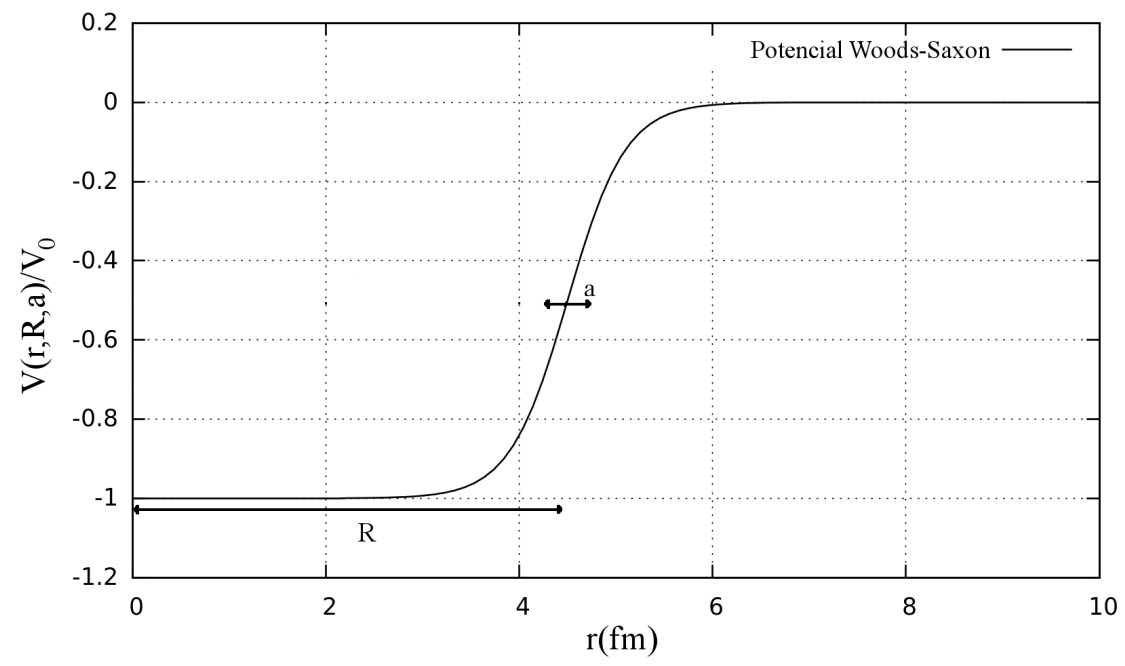

Figura 4.2: Forma do potencial de Woods-Saxon. 


\subsubsection{Potencial de São Paulo}

Outra importante parametrização muito utilizada é a baseada no potencial de convolução, que foi proposto com o objetivo de eliminar possíveis ambiguidades que surgem da utilização de potenciais fenomenológicos, como o Woods-Saxon. Nesse caso o potencial efetivo é associado à interação individual dos núcleons de cada componente da reação. Desta forma, a distribuição nuclear segue supostamente a distribuição de Fermi-Dirac. A Fig. 5.5 mostra a geometria utilizada no potencial de São Paulo.

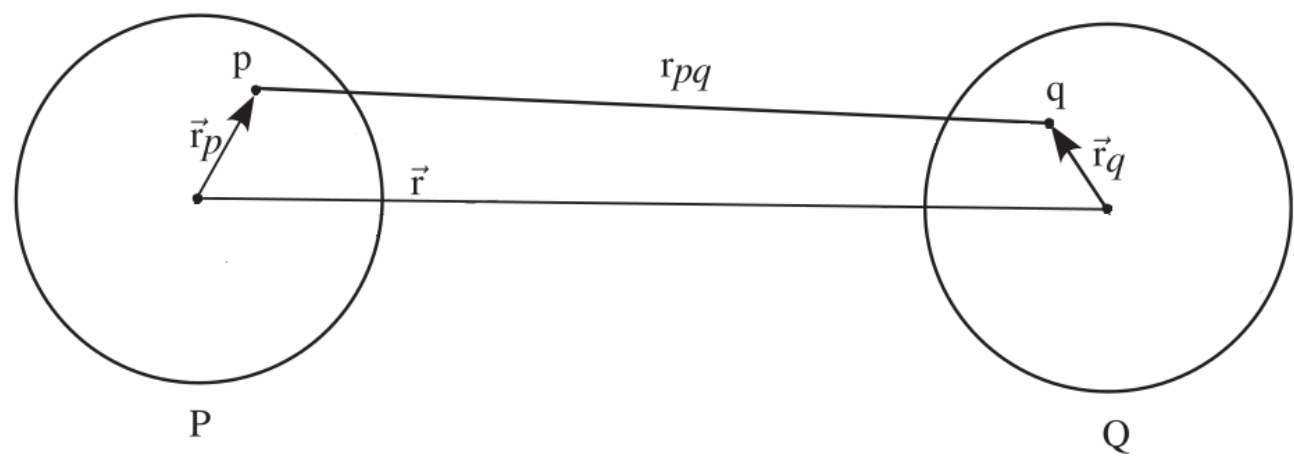

Figura 4.3: Geometria utilizada no potencial de São Paulo.

Chamon et al [35, 36, 4] desenvolveram um modelo para a interação nuclear baseado em efeitos da não-localidade de Pauli, onde a interação nuclear está relacionada com o potencial de convolução $\left(V_{S P P}\right)$ através da seguinte equação:

$$
V_{N}\left(\vec{r}_{p q}, E\right)=V_{S P P} e^{-4 v^{2} / c^{2}}
$$

onde $c$ é a velocidade da luz no vácuo e $v$ é a velocidade relativa entre os dois núcleos, que pode ser escrita como:

$$
v^{2}\left(\vec{r}_{p q}\right)=\frac{2}{\mu}\left[E-V_{C}\left(\vec{r}_{p q}\right)-V_{N}\left(\vec{r}_{p q}, E\right)\right]
$$

sendo $V_{C}$ é o potencial Coulombiano. O potencial de convolução $\left(V_{S P P}\right)$ é dado em termos da interação núcleon-núcleon e das densidades nucleares: 


$$
V_{S P P}\left(\vec{r}_{p q}\right)=\iint \rho_{P}\left(\vec{r}_{p}\right) \rho_{Q}\left(\vec{r}_{q}\right) V_{N N}\left(\vec{r}_{p q}-\vec{r}_{p}+\vec{r}_{q}\right) d \vec{r}_{p} d \vec{r}_{q}
$$

onde $\rho_{P}\left(\vec{r}_{p}\right)\left(\rho_{Q}\left(\vec{r}_{q}\right)\right)$ é a densidade nuclear dos núcleos e:

$$
V_{N N}\left(\vec{r}_{p q}\right)=\iint \rho_{m}\left(\vec{r}_{p}\right) \rho_{m}\left(\vec{r}_{q}\right) V_{0} \delta\left(\vec{r}_{p q}-\vec{r}_{p}+\vec{r}_{q}\right) d \vec{r}_{p} d \vec{r}_{q}
$$

sendo $V_{0}=-456 \mathrm{MeV} \cdot \mathrm{fm}^{3}$ e $\rho_{m}$ a densidade de matéria do núcleon.

É possível obter o potencial de São Paulo numericamente através da resolução da Eq. (4.27).

\subsection{Espalhamento Inelástico}

O processo conhecido como espalhamento inelástico ocorre quando não há troca de núcleons entre os núcleos interagentes, porém, há a redistribuição de energia da partícula incidente entre os dois núcleos. Esses espalhamentos são escritos na forma $A\left(a, a^{\prime}\right) A^{*}$, onde $a^{\prime}$ representa o mesmo núcleo de $a$, mas com energia diferente e $A^{*}$ o mesmo núcleo $A$, porém num estado excitado. Processos inelásticos podem ser interpretados através do método de canais acoplados.

\subsubsection{Método de Canais Acoplados (CC)}

Diferentemente do modelo óptico, onde a parte imaginária do potencial nuclear descreve a contribuição de possíveis canais inelásticos, o Método de Canais Acoplados inclui explicitamente alguns processos inelásticos. Desta forma, torna-se possível verificar a influência destes na seção de choque.

Considerando o espalhamento de um projétil $p$ sobre um alvo $t$, pode-se escrever a hamiltoniana do sistema como:

$$
H=T+h+\sum V
$$


onde $T$ é a energia cinética total, $h=H_{p}+H_{t}$ são as hamiltonianas internas dos projétil e alvo respectivamente e $V$ são os potenciais de interação entre cada núcleon do sistema projétil-alvo. Essa equação pode ser escrita da mesma forma para os canais elásticos e inelásticos, porém a função de onda é uma combinação linear dos estados fundamental $(\alpha)$ e excitado $\left(\alpha^{\prime}\right)$. Por simplicidade, será considerado que apenas um dos núcleos, por exemplo o projétil, é excitado na colisão e que este núcleo tem apenas um estado excitado. Assim, a função de onda pode ser escrita como:

$$
|\Psi\rangle=\chi_{\alpha}\left|\phi_{\alpha}\right\rangle+\chi_{\alpha^{\prime}}\left|\phi_{\alpha^{\prime}}\right\rangle
$$

onde $\phi_{\alpha}\left(\phi_{\alpha^{\prime}}\right)$ representa a função de onda do projétil no estado fundamental (excitado) e $\chi_{\alpha}\left(\chi_{\alpha^{\prime}}\right)$ representa o movimento relativo projétil-alvo no estado fundamental (excitado).

A equação de Schrödinger para os estados fundamental e excitado pode ser escrita como:

$$
H_{\alpha}|\Psi\rangle=E_{\alpha}|\Psi\rangle \quad H_{\alpha^{\prime}}|\Psi\rangle=E_{\alpha^{\prime}}|\Psi\rangle
$$

Empregando as Eqs. (4.29) e (4.30) na Eq. (4.31), obtém-se para o estado fundamental:

$$
\left[T_{\alpha}+h_{\alpha}+\sum V_{\alpha}\right]\left[\chi_{\alpha}\left|\phi_{\alpha}\right\rangle+\chi_{\alpha^{\prime}}\left|\phi_{\alpha^{\prime}}\right\rangle\right]=E_{\alpha}\left[\chi_{\alpha}\left|\phi_{\alpha}\right\rangle+\chi_{\alpha^{\prime}}\left|\phi_{\alpha^{\prime}}\right\rangle\right]
$$

Projetando nos estados $\left\langle\phi_{\alpha}\right|$ e usando as identidades $h_{\alpha}\left|\phi_{\alpha}\right\rangle=\varepsilon_{\alpha}\left|\phi_{\alpha}\right\rangle$ e $\left\langle\phi_{\alpha} \mid \phi_{\alpha^{\prime}}\right\rangle=$ $\delta_{\alpha \alpha^{\prime}}[37]:$

$$
\left[T_{\alpha}+\varepsilon_{\alpha}+V_{\alpha}\right] \chi_{\alpha}+\left\langle\phi_{\alpha}\left|\sum V_{\alpha} \chi_{\alpha^{\prime}}\right| \phi_{\alpha^{\prime}}\right\rangle=E_{\alpha} \chi_{\alpha}
$$


portanto:

$$
\left[E_{\alpha}-T_{\alpha}-\varepsilon_{\alpha}-V_{\alpha}\right] \chi_{\alpha}=\left\langle\phi_{\alpha}\left|\sum V_{\alpha} \chi_{\alpha^{\prime}}\right| \phi_{\alpha^{\prime}}\right\rangle
$$

onde $E_{\alpha}$ é a energia total do sistema, $T_{\alpha}$ é a energia cinética total, $\varepsilon_{\alpha}$ a soma das energias internas do projétil e alvo e $V_{\alpha}$ o potencial de interação nuclear no estado fundamental. Analogamente para a hamiltoniana do canal inelástico:

$$
\left[E_{\alpha^{\prime}}-T_{\alpha^{\prime}}-\varepsilon_{\alpha^{\prime}}-V_{\alpha^{\prime}}\right] \chi_{\alpha^{\prime}}=\left\langle\phi_{\alpha^{\prime}}\left|\sum V_{\alpha^{\prime}} \chi_{\alpha}\right| \phi_{\alpha}\right\rangle
$$

onde os termos são análogos à Eq. (4.35) para o estado excitado. Com isso obtém-se as duas equações acopladas. O número de equações acopladas será igual ao número de estados excitados introduzidos na função de onda original (Eq. (4.30)). Essas equações devem ser resolvidas numericamente.

Os termos $V_{\alpha}$ e $V_{\alpha^{\prime}}$ são de suma importância, pois é na resolução deles que são considerados os diferentes modelos de estrutura, como Modelo de Camadas, Modelo Coletivo e Modelo de Partícula Única (Cluster).

\subsubsection{Modelo de Camadas}

Nesse processo os núcleons são excitados e elevados a camadas superiores ao receberem a energia proveniente do núcleo incidente. Esse modelo explica as quantidades discretizadas de energia que são capazes de excitar o núcleo alvo e os números mágicos, onde a estabilidade do núcleo é máxima.

\subsubsection{Modelo Coletivo}

Nesse formalismo a excitação nuclear se apresenta na forma de deformações das distribuições de carga, produzindo potenciais esfericamente deformados. O raio nuclear, constante para núcleos esféricos e estáveis, passa a variar conforme a equação: 


$$
R(\theta, \phi)=R_{0}\left(1+\sum_{\lambda \mu} \alpha_{\lambda \mu} Y_{\lambda \mu}(\theta, \phi)\right)
$$

onde $R_{0}$ é o raio não deformado, $\alpha_{\lambda \mu}$ é o parâmetro de deformação nuclear e $Y_{\lambda \mu}(\theta, \phi)$ são os harmônicos esféricos.

Nota-se que a deformidade do raio pode ser representada em função dos Polinômios de Legendre e, portanto, ser tratada em termos de uma expansão multipolar, onde o termo de monopolo é o raio não deformado. A Fig. 4.4 mostra um esquema com os estados de excitação mais comuns: monopolo e quadrupolo.

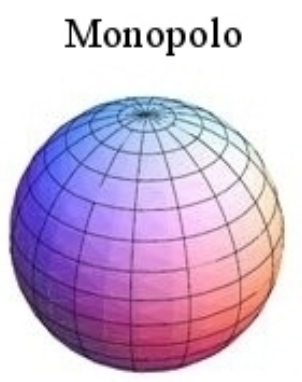

Esférico

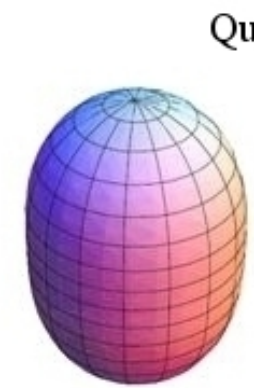

Prolato
Quadrupolo

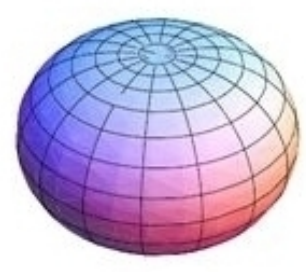

Oblato

Figura 4.4: Esquema generalizado do modelo coletivo.

\subsubsection{Modelo de Partícula Única (Cluster)}

Alguns núcleos podem ser descritos como sendo compostos por um caroço e uma partícula de valência fracamente ligada, como é o caso do ${ }^{7}$ Be. Nesta situação não são bem representados pelo modelo de excitação coletiva, mas sim pela excitação de uma partícula única. Dessa maneira, as excitações nucleares são descritas em termos da interação de cada fragmento com o alvo, sendo assim o potencial de interação entre o projétil e o alvo pode ser descrito como uma soma dos potenciais internos. A Fig. 4.5 mostra um esquema desse modelo. 


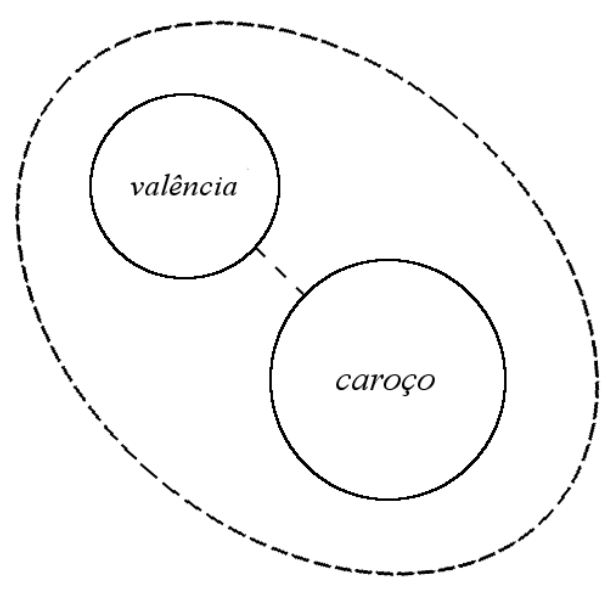

Figura 4.5: Esquema generalizado do modelo de cluster.

Considerando que o projétil possa ser descrito como o cluster de duas partículas $a$ e $b$ :

$$
V(\vec{R}, \vec{r})=V_{a}\left(\overrightarrow{R_{a}}\right)+V_{b}\left(\overrightarrow{R_{b}}\right)
$$

onde $\vec{R}$ é a coordenada do centro de massa, $\vec{r}$ é a coordenada interna entre os clusters, $V_{a}$ e $V_{b}$ são os potenciais e $R_{a}$ e $R_{b}$ são os raios dos clusters, dados por:

$$
\overrightarrow{R_{a}}=\vec{R}+\frac{m_{b}}{m_{a}+m_{b}} \vec{r} \quad \overrightarrow{R_{b}}=\vec{R}-\frac{m_{a}}{m_{a}+m_{b}} \vec{r}
$$

\subsection{Aproximação de Born de Ondas Distorcidas (DWBA)}

Existem casos onde há uma sobreposição considerável das funções de onda dos canais de entrada $(\alpha=a+A)$ e saída $(\beta=b+B)$, e, nestes casos, existem possibilidades finitas de haver uma reação de transferência devido a essa interferência dos potenciais nucleares $(b+x=a$ e $A+x=B)$. Essa interferência é, em geral, pequena o suficiente para que seja possível tratá-la como uma perturbação. A partir da Te- 
oria de Perturbações, a amplitude de transição dos dois estados pode ser descrita por $[30]$ :

$$
T_{D W B A}=J \iint d \overrightarrow{r_{\alpha}} d \overrightarrow{r_{\beta}} \chi_{\beta}^{*}\left\langle\phi_{\beta}|\Delta V| \phi_{\alpha}\right\rangle \chi_{\alpha}
$$

onde $r_{\alpha}\left(r_{\beta}\right)$ é a coordenada dos núcleos nos canais de entrada(saída), $\chi_{\alpha}\left(\chi_{\beta}\right)$ é a função de onda distorcida do canal de entrada(saída) antes da colisão, $\phi_{\alpha}\left(\phi_{\beta}\right)$ é a função de onda dos estados internos do núcleo no canal de entrada(saída) e $\Delta V$ é o potencial de transição entre os canais de entrada e saída. O elemento de matriz $\left\langle\phi_{\beta}|\Delta V| \phi_{\alpha}\right\rangle$ mostra a interferência entre os estados inicial e final e fornece a transição entre o canal de entrada e saída, sendo obtido pela expressão [38]:

$$
\left\langle\phi_{\beta}|\Delta V| \phi_{\alpha}\right\rangle=\int d \xi \psi_{b}^{*} \psi_{B}^{*} \Delta V \psi_{a} \psi_{A}
$$

onde $\xi$ são as coordenadas internas dos núcleos.

A Eq. (4.39) pode ser escrita em termos dos canais de entrada (prior) e saída (post). Para isso, o potencial de transição é escrito como função do potenciais de interação entre os caroços ( $b$ e $A$ ) e da interação da partícula de valência com esses caroços:

$$
\Delta V_{\alpha(\beta)}=V_{x A(b x)}+U_{b A}-U_{\alpha(\beta)}
$$

onde $V_{x A(b x)}$ são os potenciais de ligação da partícula $x$ no núcleo $A$ e $b$, respectivamente, $U_{b A}$ é o potencial core-core e $U_{\alpha(\beta)}$ são os potenciais ópticos de espalhamento. Desta forma, a amplitude de transição pode ser escrita como função dos canais $\alpha$ e $\beta$ :

$$
\begin{aligned}
T_{D W B A}^{\text {prior }} & =\iint d \overrightarrow{r_{\alpha}} d \overrightarrow{r_{\beta}} \chi_{\beta}^{*}\left\langle\phi_{\beta}\left|V_{x A}+U_{b A}-U_{\alpha}\right| \phi_{\alpha}\right\rangle \chi_{\alpha} \\
T_{D W B A}^{p o s t} & =\iint d \overrightarrow{r_{\alpha}} d \overrightarrow{r_{\beta}} \chi_{\beta}^{*}\left\langle\phi_{\beta}\left|V_{b x}+U_{b A}-U_{\beta}\right| \phi_{\alpha}\right\rangle \chi_{\alpha}
\end{aligned}
$$


A seção de choque de transferência na aproximação DWBA é calculada por:

$$
\frac{d \sigma}{d \Omega}=\frac{\mu_{\alpha} \mu_{\beta}}{\left(2 \pi \hbar^{2}\right)^{2}} \frac{\kappa_{\beta}}{\kappa_{\alpha}} \frac{1}{\left(2 J_{A}+1\right)\left(2 J_{a}+1\right)} \sum_{m_{a}}\left|T_{D W B A}\right|^{2}
$$

As funções de onda distorcidas $\chi_{\alpha}$ e $\chi_{\beta}$ podem ser obtidas pela resolução da equação de Schrödinger, como vista na Eq. (4.1).

Para obter a amplitude de transição é necessário conhecer as funções de onda internas dos núcleos $\left(\psi_{a}, \psi_{A}, \psi_{b}\right.$ e $\left.\psi_{B}\right)$. Uma boa modelagem para o cálculo é considerar o núcleo como sendo formado por um caroço nuclear e uma partícula de valência, ou cluster, que será transferida de um núcleo para o outro. A partícula de valência pode ser considerada em um estado de single-particle acoplado aos diversos possíveis estados do caroço, portanto a função de onda do estado ligado será uma combinação linear entre todos os estados acessíveis ao caroço $\left(\psi_{C}^{J_{C}}\right)$ e a partícula de valência $\left(\phi_{l s j}\right)$.

$$
\Psi_{J M}(\xi, \vec{r})=\frac{1}{\sqrt{n}} \sum_{l, j, J} A_{l s j}^{j J J_{c}}\left[\psi_{C}^{J_{C}}(\xi) \otimes \phi_{l s j}(\vec{r})\right]
$$

onde $n$ é o número de clusters no estado ligado, que são iguais à partícula de valência. O estado ligado tem momento angular $J$ e projeção $M$, o caroço possui spin $J_{C}$ e $l, s$ e $j$ são os números quânticos angulares da partícula de valência. O termo

$A_{l s j}^{j J J_{c}}$ é conhecido como amplitude espectroscópica, onde seu módulo ao quadrado é a probabilidade de encontrar a partícula de valência ligada ao caroço com estados definidos $\left(l, s, j\right.$ e $\left.J_{C}\right)$, também conhecido como fator espectroscópico:

$$
S F_{l s j}^{j J J_{c}}=\left|A_{l s j}^{j J J_{c}}\right|^{2}
$$

\subsubsection{Transferência Elástica}

Nesse tipo de reação acontece a transferência de alguns núcleons entre o projétil e o alvo durante o processo. A reação de pick-up ocorre quando há transferência de núcleons do alvo para o projétil, enquanto a reação de stripping ocorre quando a 
transferência acontece do projétil para o alvo. Um importante evento desse tipo é a reação de transferência elástica [39]. Onde os canais de entrada e saída são iguais:

$$
A+a \rightarrow a+A
$$

onde $A=a+x$, sendo $x$ a partícula trocada entre o projétil e o alvo. O canal de saída desse processo é o mesmo já mencionado no espalhamento elástico, portanto, não é possível separar experimentalmente as duas contribuições. A Fig. 4.6 mostra um esquema como comparação dos dois tipos de reação.

(a)

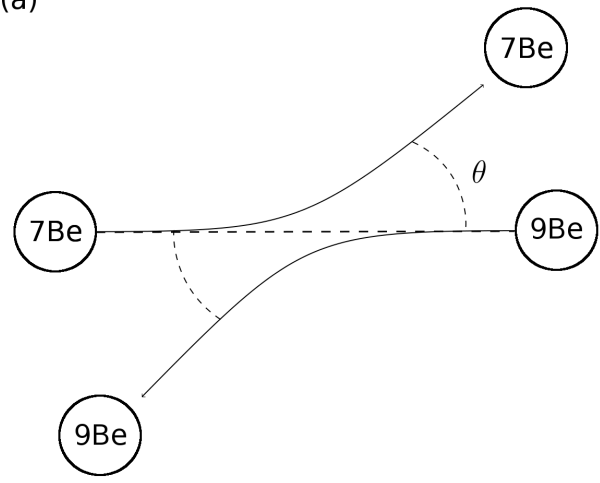

(b)

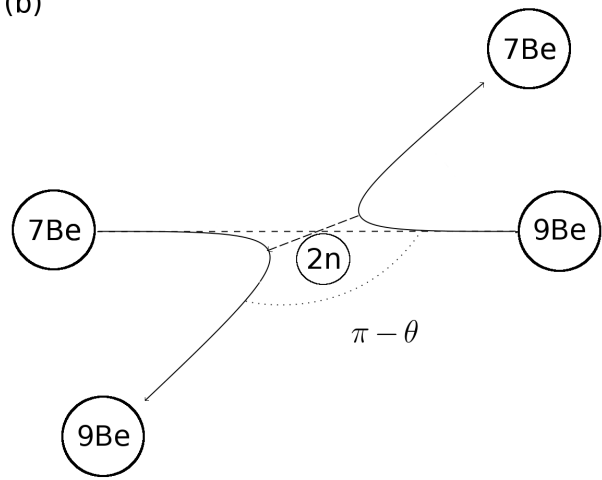

Figura 4.6: Relação cinemática do (a) espalhamento elástico e (b) transferência elástica, ambos no referencial do centro de massa.

Utilizando as leis de conservação de momento e energia é possível obter uma relação entre os ângulos de espalhamento no centro de massa:

$$
\theta_{A}+\theta_{a}=\pi
$$

Devido ao fato de ser impossível separar as contribuições elásticas e de transferência elástica, a amplitude de espalhamento é obtida pela soma das duas parcelas. Portanto, a seção de choque pode ser escrita pela equação:

$$
\frac{d \sigma}{d \Omega}=\left|f_{e l}(\theta)+f_{t r}(\pi-\theta)\right|^{2}
$$


onde $f_{e l}(\theta)$ refere-se ao espalhamento elástico, calculada pelo modelo óptico, e $f_{t r}(\pi-\theta)$ à transferência elástica, calculada pela teoria DWBA. 


\section{Capítulo 5}

\section{Análise da Distribuição ${ }^{7} \mathrm{Be}+{ }^{9} \mathrm{Be}$}

Nesse capítulo serão apresentadas as análises da distribuição angular ${ }^{7} \mathrm{Be}+{ }^{9} \mathrm{Be}$ realizadas com os formalismos de Modelo Óptico, Canais Acoplados e Aproximação de Born de Ondas Distorcidas. A análise da qualidade dos potenciais obtidos e comparações com estudos já realizados para esse sistema também serão vistas. Todos os cálculos foram realizados utilizando o código computacional FRESCO [40]. Os Inputs utilizados nos cálculos estão exemplificados no Apêndice A.

\subsection{Cálculos com Modelo Óptico}

O modelo óptico é um importante formalismo utilizado para descrever distribuições angulares experimentais. Neste, a parte real do potencial descreve somente o espalhamento elástico, fornecendo importantes informações sobre a geometria nuclear, enquanto a parte imaginária representa todos os canais não elásticos.

Para este cálculo foram utilizados potenciais superficiais e volumétricos, bem como fatores de forma de Woods-Saxon e do Potencial de São Paulo.

\subsubsection{Potencial de Woods-Saxon}

Com o fator de forma de Woods-Saxon (WS) (Eq. (4.23)) foram utilizados diferentes conjuntos de potenciais ópticos encontrados na literatura para o sistema similar ${ }^{7} \mathrm{Li}+{ }^{9} \mathrm{Be}$ nas energias entre 24 e $34 \mathrm{MeV}[3,41]$ e para o sistema ${ }^{7} \mathrm{Be}+{ }^{9} \mathrm{Be}$ 
nas energias de $21 \mathrm{MeV}$ [1] e 23,7 MeV [2]. A Tabela 5.1 apresenta os parâmetros dos potenciais utilizados nos cálculos e a Fig. 5.1 mostra os resultados obtidos.

Tabela 5.1: Parâmetros dos potenciais ópticos, com fator de forma Woods-Saxon, utilizados nos cálculos. Tipo identifica os potenciais volumétricos (Vol.) e superficiais (Sup.). Os valores dos raios reduzidos foram convertidos em raio absoluto usando as relações $R=r_{0}\left(A_{T}^{1 / 3}+A_{P}^{1 / 3}\right)^{1}$ e $N$ é o número de dados.

\begin{tabular}{cccccccccc}
\hline Conj. & $V$ & $r_{0}$ & $a$ & $W$ & $r_{i}$ & $a_{i}$ & Tipo & $\chi^{2} / N$ & Ref. \\
& $(\mathrm{MeV})$ & $(\mathrm{fm})$ & $(\mathrm{fm})$ & $(\mathrm{MeV})$ & $(\mathrm{fm})$ & $(\mathrm{fm})$ & & & \\
\hline (a) & 60,0 & 0,67 & 0,88 & 17,40 & 1,86 & 0,78 & Sup. & 272,7 & {$[3]$} \\
(b) & 152,0 & 0,72 & 0,75 & 6,72 & 2,72 & 0,76 & Vol. & 62,1 & {$[3]$} \\
(c) & 30,0 & 1,20 & 0,57 & 7,10 & 2,71 & 0,79 & Vol. & 56,2 & {$[3]$} \\
(d) & 73,1 & 1,19 & 0,78 & 8,90 & 2,52 & 0,92 & Vol. & 16,9 & {$[41]$} \\
(e) & 34,4 & 1,21 & 0,76 & 8,90 & 2,43 & 1,02 & Vol. & 5,1 & {$[41]$} \\
(f) & 23,0 & 2,15 & 0,61 & 10,30 & 1,89 & 1,13 & Vol. & 71,5 & {$[41]$} \\
(g) & 48,6 & 1,28 & 0,88 & 12,13 & 1,86 & 0,78 & Vol. & 115,9 & {$[2]$} \\
(h) & 12,1 & 2,30 & 0,57 & 7,51 & 2,71 & 0,79 & Vol. & 80,8 & {$[2]$} \\
(i) & 30,0 & 0,83 & 0,73 & $0,5+\mathrm{E}_{\text {lab }}$ & 0,92 & 0,75 & Vol. & 12,2 & {$[1]$} \\
(j) & 60,0 & 0,50 & 0,88 & 24,40 & 0,74 & 0,68 & Sup. & 37,7 & {$[1]$} \\
\hline
\end{tabular}

Através do valor obtido para o $\chi^{2} / N$ observa-se que alguns dos parâmetros obtidos da literatura estão em bom acordo com os dados, como é o caso do potencial (e), como pode ser visto na Fig. 5.1.

\footnotetext{
${ }^{1}$ Onde $A_{T}$ e $A_{P}$ representam o número de massa do alvo e projétil respectivamente.
} 

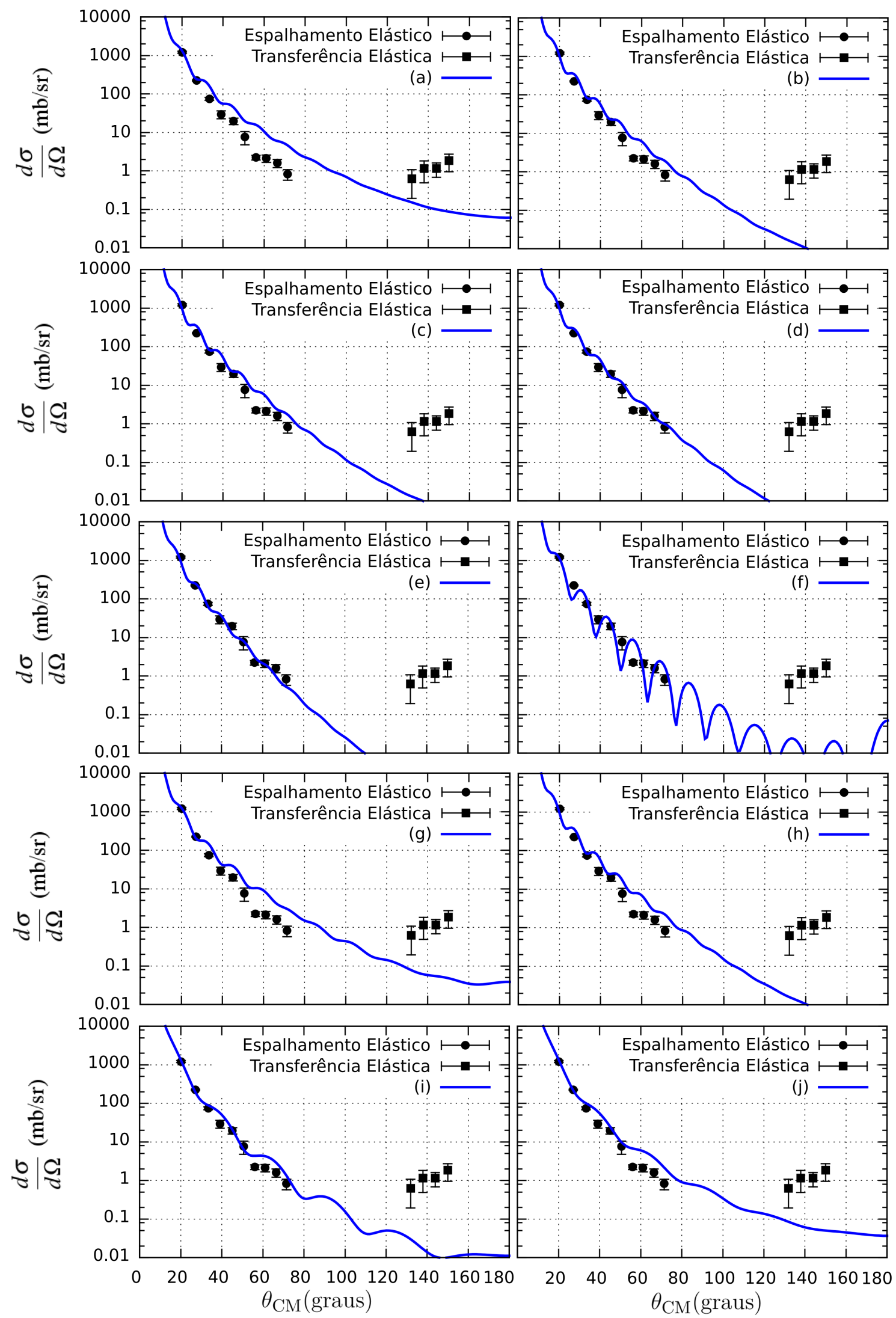

Figura 5.1: Distribuição angular do espalhamento quasielástico ${ }^{7} \mathrm{Be}+{ }^{9} \mathrm{Be}$ para $\mathrm{E}_{\text {lab }}=23,1 \mathrm{MeV}$ comparada com cálculos de modelo óptico utilizando fator de forma Woods-Saxon. As identificações de (a) a (j) se referem aos potenciais apresentados na Tabela 5.1. 
Para otimizar a comparação entre os dados e os cálculos, foram feitos ajustes nos potenciais, variando-se as profundidades real $(V)$ e imaginária $(W)$, e fixando a geometria $\left(r\right.$ e $a$ ) afim de obter o menor valor de $\chi^{2} / N$. Os parâmetros dos potenciais obtidos neste processo podem ser vistos na Tabela 5.2. Os resultados obtidos são apresentados na Fig. 5.2.

Tabela 5.2: Parâmetros do potencial óptico, com fator de forma Woods-Saxon, obtidos com a variação das profundidades $V$ e $W$, minimizando o valor de $\chi^{2} / N$. Os valores dos raios reduzidos foram convertidos em raio absoluto usando a relação

\begin{tabular}{ccccccccc}
$R=r_{0}\left(A_{T}^{1 / 3}+A_{P}^{1 / 3}\right)$. & \multicolumn{1}{c}{. } \\
\hline Conj. & $V$ & $r_{0}$ & $a$ & $W$ & $r_{i}$ & $a_{i}$ & Tipo & $\chi^{2} / N$ \\
& $(\mathrm{MeV})$ & $(\mathrm{fm})$ & $(\mathrm{fm})$ & $(\mathrm{MeV})$ & $(\mathrm{fm})$ & $(\mathrm{fm})$ & & \\
\hline (a) & $74,6(1)$ & 0,67 & 0,88 & $4,8(1)$ & 1,86 & 0,78 & Sup. & 33,3 \\
(b) & $145,8(1)$ & 0,72 & 0,75 & $3,5(1)$ & 2,72 & 0,76 & Vol. & 2,3 \\
(c) & $28,4(1)$ & 1,20 & 0,57 & $3,7(1)$ & 2,71 & 0,79 & Vol. & 2,6 \\
(d) & $72,5(1)$ & 1,19 & 0,78 & $5,5(1)$ & 2,52 & 0,92 & Vol. & 2,3 \\
(e) & $47,2(1)$ & 1,21 & 0,76 & $7,3(1)$ & 2,43 & 1,02 & Vol. & 3,2 \\
(f) & $48,6(119)$ & 2,15 & 0,61 & $28,4(42)$ & 1,89 & 1,13 & Vol. & 5,4 \\
(g) & $47,9(3)$ & 1,28 & 0,88 & $6,7(1)$ & 1,86 & 0,78 & Vol. & 24,9 \\
(h) & $14,7(3)$ & 2,30 & 0,57 & $3,4(1)$ & 2,71 & 0,79 & Vol. & 9,1 \\
(i) & $19,7(8)$ & 0,83 & 0,73 & $13,7(9)$ & 0,92 & 0,75 & Vol. & 4,1 \\
(j) & $66,9(23)$ & 0,50 & 0,88 & $11,9(8)$ & 0,74 & 0,68 & Sup. & 5,8 \\
\hline
\end{tabular}

A mesma metodologia já havia sido feita anteriormente na análise dos dados obtidos por Mukha et al [2], onde foram utilizados alguns dos potenciais da literatura utilizados neste trabalho e através da variação das profundidades real e imaginária foram obtidos parâmetros bastante parecidos com o Conj. (e). 

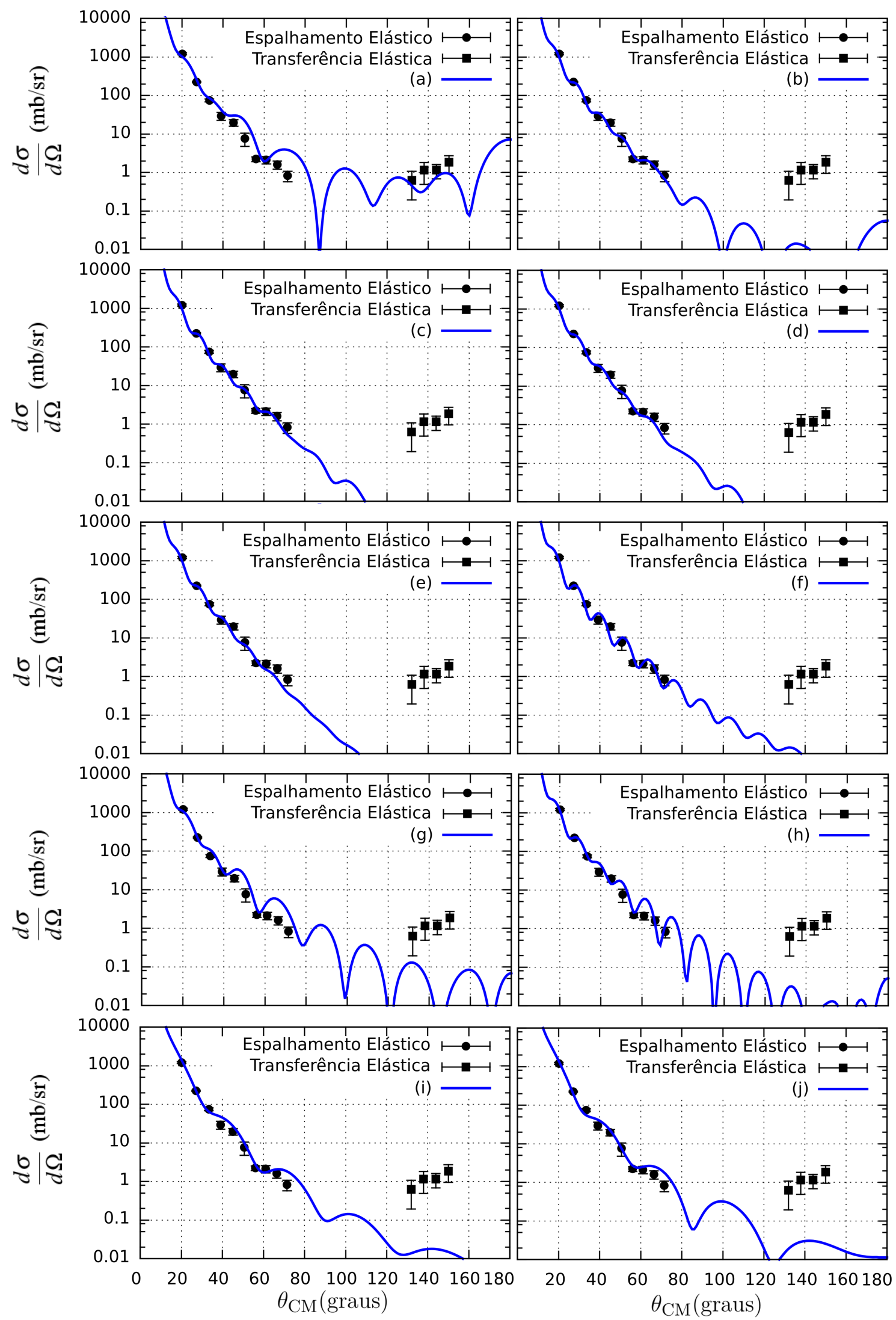

Figura 5.2: Distribuição angular do espalhamento quasielástico ${ }^{7} \mathrm{Be}+{ }^{9} \mathrm{Be}$ para $\mathrm{E}_{\text {lab }}=23,1 \mathrm{MeV}$ comparada com cálculos de modelo óptico utilizando fator de forma Woods-Saxon, variando as profundidades real e imaginária. As identificações de (a) a (j) se referem aos potenciais apresentados na Tabela 5.2. 
Como é possível observar nos gráficos apresentados na Fig. 5.2, após o ajuste das profundidades, alguns potenciais descrevem razoavelmente bem os dados, porém ainda não reproduzem integralmente as oscilações existentes.

Desta forma, todos os parâmetros do potencial Woods-Saxon foram deixados livres para que o código computacional SFRESCO busque os valores dos seis parâmetros que minimize o $\chi^{2} / N$. Os parâmetros do potencial obtidos neste processo podem ser vistos na Tabela 5.3. Os resultados estão apresentados na Fig. 5.3.

Tabela 5.3: Parâmetros do potencial óptico, com fator de forma Woods-Saxon, obtidos pela variação de todos os parâmetros, minimizando o valor de $\chi^{2} / N$. Os valores dos raios reduzidos foram convertidos em raio absoluto usando a relação

\begin{tabular}{ccccccccc}
$R=$ & $r_{0}\left(A_{T}^{1 / 3}+A_{P}^{1 / 3}\right)$. \\
\hline Conj. & $V$ & $r_{0}$ & $a$ & $W$ & $r_{i}$ & $a_{i}$ & Tipo & $\chi^{2} / N$ \\
& $(\mathrm{MeV})$ & $(\mathrm{fm})$ & $(\mathrm{fm})$ & $(\mathrm{MeV})$ & $(\mathrm{fm})$ & $(\mathrm{fm})$ & & \\
\hline$(\mathrm{k})$ & $42(15)$ & $0,6(2)$ & $0,6(1)$ & $11(5)$ & $0,4(2)$ & $1,2(2)$ & Sup. & 0,4 \\
$(\mathrm{l})$ & $43(14)$ & $0,6(2)$ & $0,6(1)$ & $14(9)$ & $0,7(3)$ & $1,2(2)$ & Vol. & 0,4 \\
\hline
\end{tabular}

O fator de forma Woods-Saxon é muito utilizado para ajustar seções de choque experimentais de espalhamento elástico, porém, devido ao grande número de parâmetros, torna-se possível ajustar diversos conjuntos de dados. A parte real do potencial óptico representa o canal elástico da reação, enquanto a parte imaginária representa todos os canais não elásticos de reação possíveis, ocultando, dessa forma, a importância de cada reação não elástica na perda do fluxo inicial de partículas e, portanto, na seção de choque total de reação. 

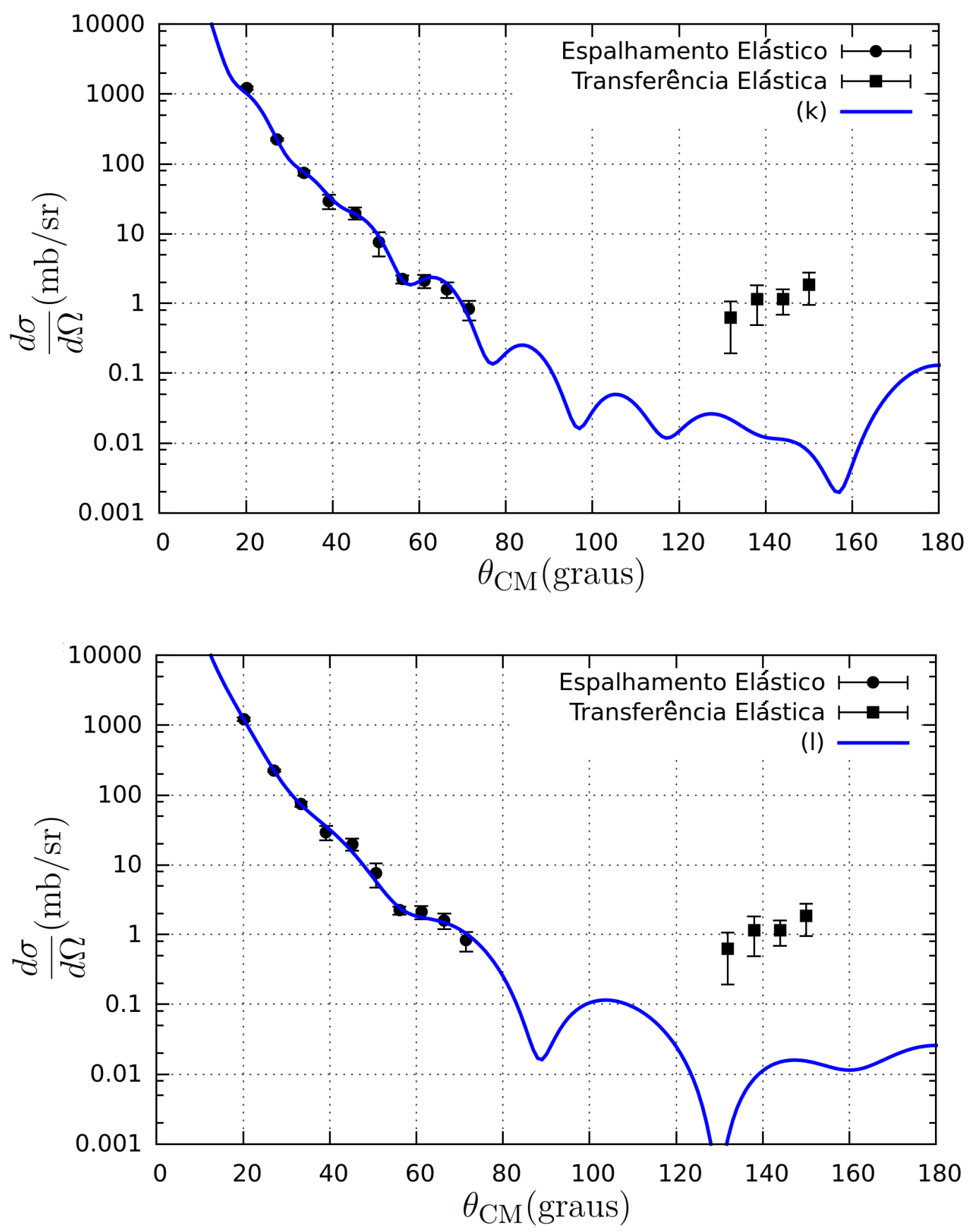

Figura 5.3: Distribuição angular do espalhamento quasielástico ${ }^{7} \mathrm{Be}+{ }^{9} \mathrm{Be}$ para $\mathrm{E}_{\text {lab }}=23,1 \mathrm{MeV}$ comparada com os cálculos de modelo óptico utilizando fator de forma Woods-Saxon, variando todos os parâmetros. As identificações (k) e (l) se referem aos potenciais apresentados na Tabela 5.3. 


\subsubsection{Potencial de São Paulo}

Além dos cálculos realizados utilizando o fator de forma de Woods-Saxon, foram realizados cálculos utilizando o Potencial de São Paulo (SPP), através do código computacional SPOMC [42] que calcula numericamente o potencial para o espalhamento elástico estudado. Os valores obtidos para o potencial de São Paulo foram utilizados pelo código FRESCO para o cálculo das seções de choque ${ }^{1}$. Os cálculos realizados foram feitos com densidades nucleares calculadas pelo código SPOMC, que assume a distribuição de Fermi-Dirac, podendo não ser realistas para o caso de núcleos radioativos. O potencial obtido pelo potencial de São Paulo pode ser visto na Fig. 5.4.

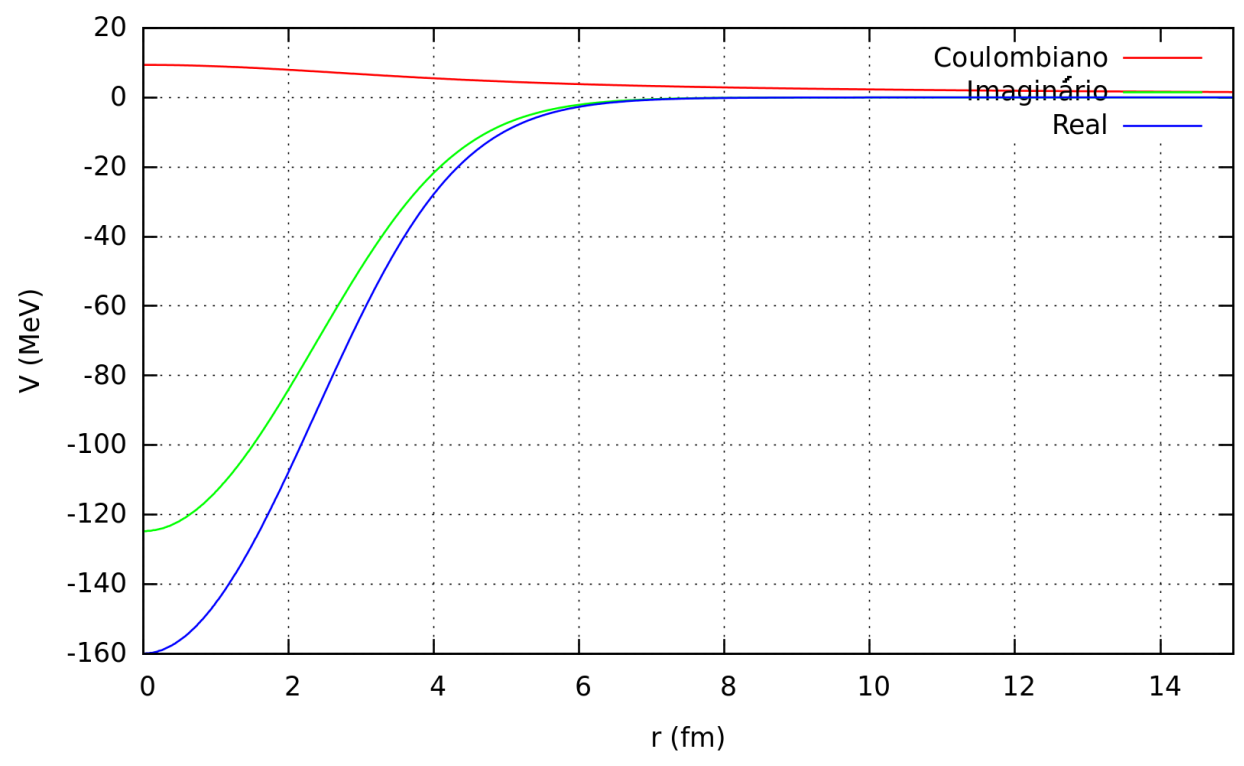

Figura 5.4: Potenciais obtidos com o código SPOMC.

No potencial de São Paulo, o potencial óptico é parametrizado apenas pelos valores da normalização real $\left(\mathrm{N}_{\mathbb{R}}\right)$ e imaginária $\left(\mathrm{N}_{\mathbb{I}}\right)$, representando um parâmetro de ajuste em relação aos dados experimentais, já que os raios nucleares e a difusividade superficial são valores predefinidos. Essas normalizações têm como valores base $\mathrm{N}_{\mathbb{R}}$ $=1,0$ e $\mathrm{N}_{\mathbb{I}}=0,78$; isso significa que para que a reação possa ser descrita com esse tipo de potencial, as normalizações obtidas devem ser próximas a esses valores. O uso do

\footnotetext{
${ }^{1} \mathrm{O}$ código SPOMC realiza os cálculos para a seção de choque, porém o FRESCO foi escolhido para essa tarefa para que fosse utilizada a mesma metodologia nos cálculos.
} 
potencial de São Paulo tem reproduzido os dados experimentais de espalhamentos elásticos com relativa precisão [4].

A busca pelas normalizações pode ser feita usando o código SFRESCO, que obtém os mínimos locais do valor de $\chi^{2} / N$. Primeiramente os cálculos foram feitos mantendo-se as normalizações fixas (a), então fixou-se $\mathrm{N}_{\mathbb{R}}=1$ enquanto $\mathrm{N}_{\mathbb{I}}$ era variado (b), em seguida fixou-se $\mathrm{N}_{\mathbb{I}}=0,78$ enquanto $\mathrm{N}_{\mathbb{R}}$ era variado (c), e numa etapa final, variou-se os dois parâmetros $((\mathrm{d})$ e (e)). Nesta última, foram obtidos dois mínimos ao redor dos valores base. A Tabela 5.4 apresenta os valores obtidos para as normalizações e para o $\chi^{2} / N$. Na Fig. 5.5 podem ser vistos os resultados obtidos com a utilização do Potencial de São Paulo.

Tabela 5.4: Parâmetros do potencial óptico, usando como fator de forma o potencial de São Paulo, obtidos pela variação das normalizações $\mathrm{N}_{\mathbb{R}}$ e $\mathrm{N}_{\mathbb{I}}$, minimizando o valor local do $\chi^{2}$.

\begin{tabular}{cccc}
\hline Conj. & $\mathrm{N}_{\mathbb{R}}$ & $\mathrm{N}_{\mathbb{I}}$ & $\chi^{2} / N$ \\
\hline (a) & 1,00 & 0,78 & 52,4 \\
(b) & 1,00 & $1,07(5)$ & 49,2 \\
(c) & $2,82(5)$ & 0,78 & 20,3 \\
(d) & $0,19(1)$ & $0,21(1)$ & 2,6 \\
(e) & $2,602(5)$ & $1,728(5)$ & 14,8 \\
\hline
\end{tabular}

Pode-se observar que alguns dos cálculos teóricos representam bem os dados. Com a variação dos valores das normalizações nota-se que o melhor ajuste foi obtido quando as normalizações são da ordem de 5 vezes menores que o previsto pelo código (ver Conj. (d) na Tabela 5.4 e Fig. 5.5(d)). Isso significa que esse potencial não é um bom modelo para esse sistema. Essa divergência pode ser devido a dois fatores que não foram considerados durante os cálculos teóricos até então: a densidade nuclear anômala do núcleo de ${ }^{7} \mathrm{Be}$ (pode ser representado como um cluster de ${ }^{4} \mathrm{He}+{ }^{3} \mathrm{He}$ com energia de ligação de 1,586 MeV), e a perda de fluxo por algum canal inelástico que não está sendo levado em consideração no cálculo. 

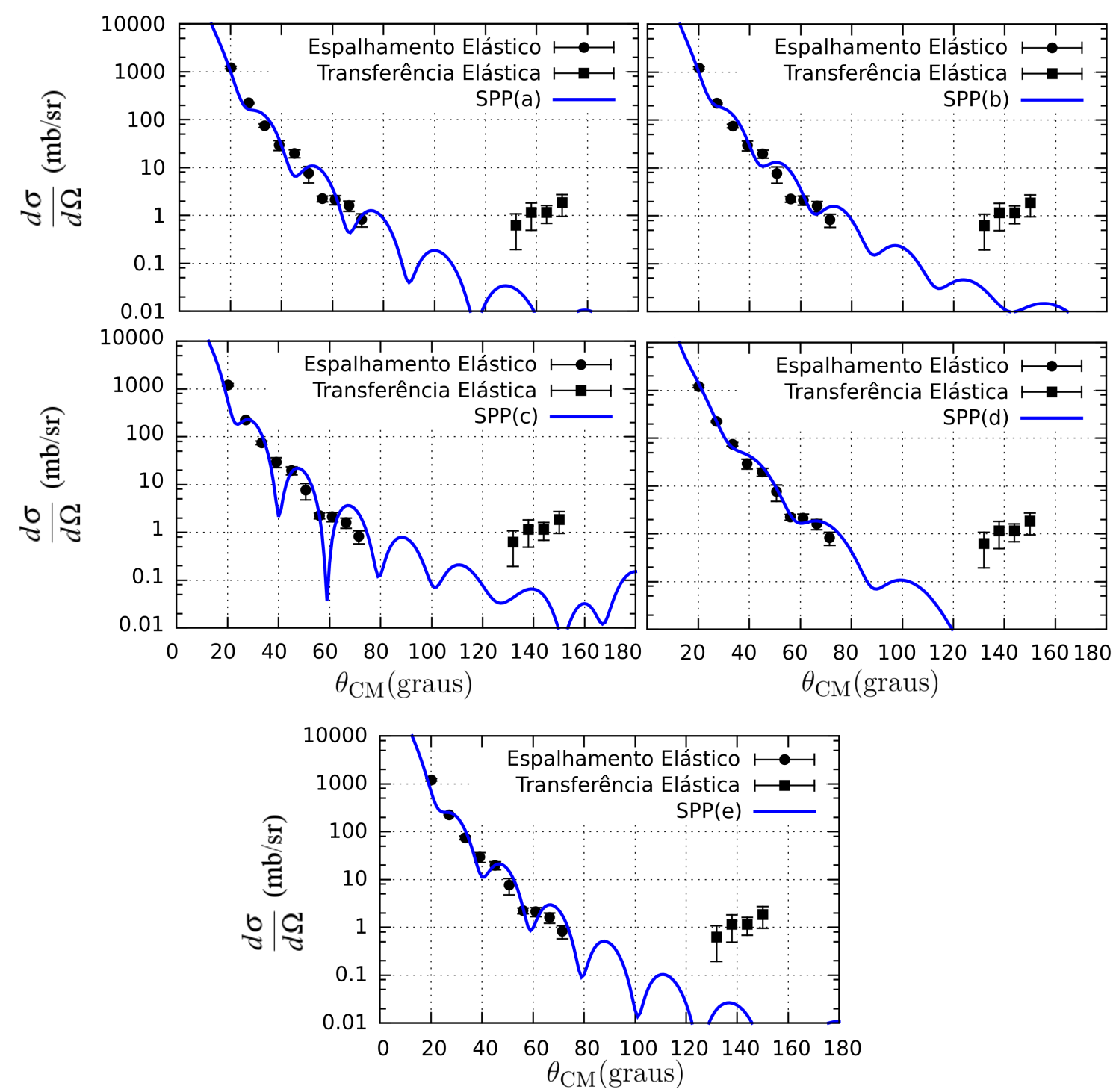

Figura 5.5: Distribuição angular do espalhamento quasielástico ${ }^{7} \mathrm{Be}+{ }^{9} \mathrm{Be}$ para $\mathrm{E}_{\text {lab }}=23,1 \mathrm{MeV}$ comparada com os cálculos realizados com o modelo óptico utilizando o potencial de São Paulo. 


\subsubsection{Potencial Misto}

Os potenciais obtidos até agora são de curto alcance na parte real e com termos rasos e de longo alcance na parte imaginária. Nessa etapa, foi utilizado um potencial misto, onde para a parte real foi utilizado o potencial de São Paulo e para a parte imaginária foi utilizado Woods-Saxon. A normalização da parte real foi mantida fixa, enquanto os parâmetros $\mathrm{W}, \mathrm{r}_{i}$ e $\mathrm{a}_{i}$ foram variados para obter o menor valor de $\chi^{2} / N$. A Tabela 5.5 mostra os parâmetros dos potenciais obtidos. A Fig. 5.6 mostra os resultados obtidos.

Tabela 5.5: Parâmetros do potencial óptico, usando o potencial de São Paulo na parte real e Woods-Saxon na parte imaginária.

\begin{tabular}{ccccc}
\hline $\mathrm{N}_{\mathbb{R}}$ & $\mathrm{W}$ & $\mathrm{r}_{i}$ & $\mathrm{a}_{i}$ & $\chi^{2} / N$ \\
\hline 1,0 & $20(4)$ & $1,10(3)$ & $1,12(3)$ & 6,0 \\
\hline
\end{tabular}

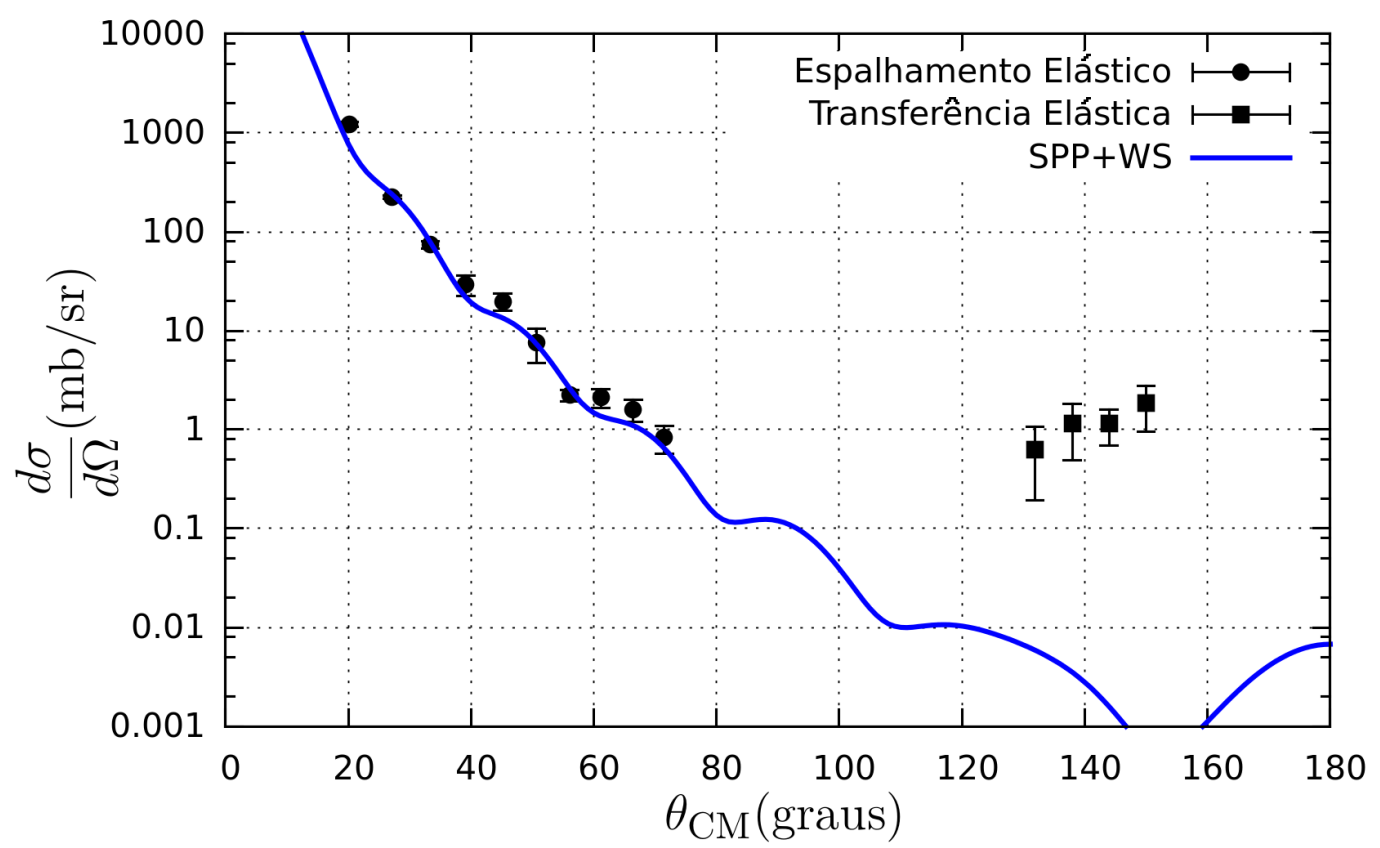

Figura 5.6: Distribuição angular do espalhamento quasielástico ${ }^{7} \mathrm{Be}+{ }^{9} \mathrm{Be}$ para $\mathrm{E}_{\text {lab }}=23,1 \mathrm{MeV}$ comparada com os cálculos de modelo óptico utilizando o potencial de São Paulo na parte real e Woods-Saxon na parte imaginária.

Nota-se que esse potencial consegue reproduzir melhor as oscilações, mostrando 
que é um bom método para descrever os dados experimentais para esse sistema.

\subsection{Cálculos com Canais Acoplados (CC)}

$\mathrm{O}$ primeiro estado excitado do núcleo de ${ }^{7} \mathrm{Be}$ tem energia de $\mathrm{E}_{x}=0,429 \mathrm{MeV}$, abaixo da resolução dos detectores utilizados, portanto, se faz necessário uma análise mais detalhada dos canais não elásticos. Através do método de Canais Acoplados (CC) é possível incluir explicitamente o primeiro estado excitado do ${ }^{7} \mathrm{Be}\left(\mathrm{J}^{\pi}=1 / 2^{-}\right)$ e com isso obter a contribuição desse canal inelástico. Esse tipo de espalhamento, onde não é possível separar os canais elásticos e inelásticos devido à resolução experimental, é conhecido como espalhamento quasielástico.

Neste cálculo, o núcleo ${ }^{7} \mathrm{Be}$ foi considerado como um sistema de dois corpos $\left({ }^{3} \mathrm{He}+{ }^{4} \mathrm{He}\right)$ através do modelo de cluster. Desta forma, adiciona-se a interação dos fragmentos ${ }^{4} \mathrm{He}+{ }^{3} \mathrm{He},{ }^{4} \mathrm{He}+{ }^{9} \mathrm{Be} \mathrm{e}{ }^{3} \mathrm{He}+{ }^{9} \mathrm{Be}$, além de incluir explicitamente o primeiro estado excitado do núcleo ${ }^{7} \mathrm{Be}$. Informações a respeito dos potenciais dos fragmentos foram obtidas da literatura [43, 44, 45]. Foram realizados cálculos com todas as combinações de potenciais possíveis. A Tabela 5.6 apresenta os parâmetros dos potenciais usados nos cálculos ${ }^{2}$.

Tabela 5.6: Parâmetros dos potenciais utilizados para o cálculo do espalhamento quasielástico. Para o potencial ${ }^{4} \mathrm{He}+{ }^{3} \mathrm{He}$, assim como a parte imaginária do potencial $\left({ }^{4} \mathrm{He}+{ }^{9} \mathrm{Be}\right)_{b}$, foi usada um potencial na forma Gaussiana: $V(r)=-V_{0} e^{-\left(r / r_{0}\right)^{2}}$.

\begin{tabular}{ccccccccccc}
\hline Sistema & $\begin{array}{c}V_{0} \\
(\mathrm{MeV})\end{array}$ & $\begin{array}{c}r_{0} \\
(\mathrm{fm})\end{array}$ & $\begin{array}{c}a_{0} \\
(\mathrm{fm})\end{array}$ & $\begin{array}{c}W_{i} \\
(\mathrm{MeV})\end{array}$ & $\begin{array}{c}r_{i} \\
(\mathrm{fm})\end{array}$ & $\begin{array}{c}a_{i} \\
(\mathrm{fm})\end{array}$ & $\begin{array}{c}V_{\text {so }} \\
(\mathrm{MeV})\end{array}$ & $\begin{array}{c}r_{\text {so }} \\
(\mathrm{fm})\end{array}$ & $\begin{array}{c}a_{\text {so }} \\
(\mathrm{fm})\end{array}$ & $\begin{array}{c}\text { Ref. } \\
\end{array}$ \\
\hline${ }^{4} \mathrm{He}+{ }^{3} \mathrm{He}$ & 83,8 & 2,52 & - & - & - & - & 0,63 & 2,52 & - & {$[43,44]$} \\
\hline$\left({ }^{4} \mathrm{He}+{ }^{9} \mathrm{Be}\right)_{a}$ & 109,0 & 1,60 & 0,64 & 22,0 & 1,60 & 0,64 & - & - & - & {$[45]$} \\
$\left({ }^{4} \mathrm{He}+{ }^{9} \mathrm{Be}\right)_{b}$ & 171,0 & 1,20 & 0,51 & 18,0 & 1,20 & 1,99 & 5,50 & 1,20 & 0,51 & {$[45]$} \\
$\left({ }^{4} \mathrm{He}+{ }^{9} \mathrm{Be}\right)_{c}$ & 141,2 & 1,16 & 0,76 & 16,4 & 1,54 & 0,99 & - & - & - & {$[45]$} \\
\hline$\left({ }^{3} \mathrm{He}+{ }^{9} \mathrm{Be}\right)_{I}$ & 54,1 & 1,85 & 0,58 & 4,2 & 1,85 & 0,58 & 2,60 & 1,85 & 0,58 & {$[45]$} \\
$\left({ }^{3} \mathrm{He}+{ }^{9} \mathrm{Be}\right)_{I I}$ & 57,0 & 1,85 & 0,58 & 4,8 & 1,85 & 0,58 & 2,40 & 1,85 & 0,58 & {$[45]$} \\
\hline
\end{tabular}

\footnotetext{
${ }^{2}$ Os mesmos potenciais foram utilizados para os potenciais de entrada e saída no FRESCO.
} 
O código FRESCO requere a criação de partições, que determinam os canais de reação e estados excitados que serão considerados no cálculo. Foram incluídas duas partições, uma para a reação ${ }^{9} \mathrm{Be}\left({ }^{7} \mathrm{Be},{ }^{7} \mathrm{Be}\right){ }^{9} \mathrm{Be}$ e outra para o chamado core-target, que é a reação do maior fragmento do projétil com os núcleons restantes, formando a reação ${ }^{12} \mathrm{C}\left({ }^{4} \mathrm{He},{ }^{7} \mathrm{Be}\right){ }^{9} \mathrm{Be}$. A Fig. 5.7 mostra os resultados obtidos com o cálculo de canais acoplados.

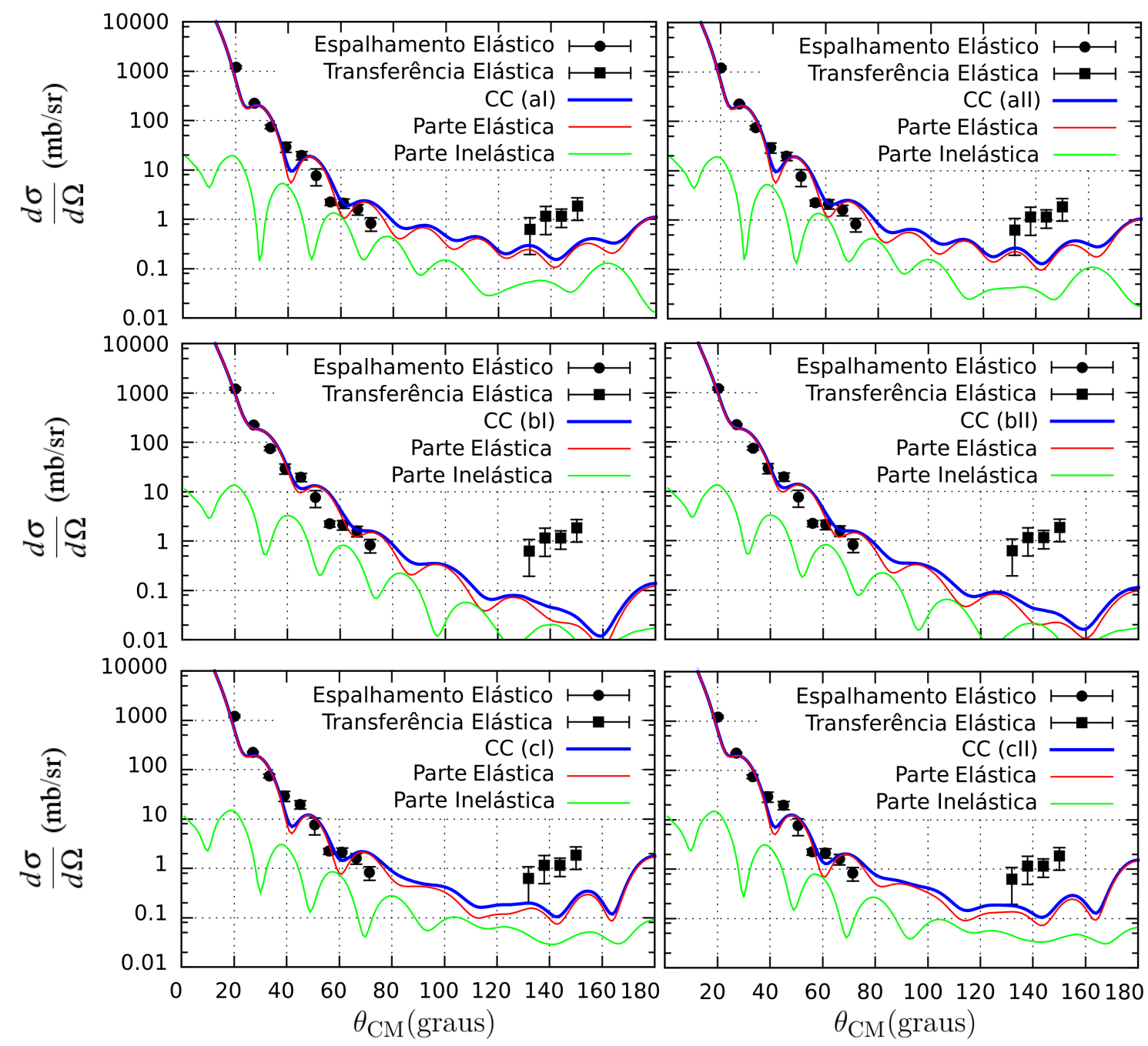

Figura 5.7: Distribuição angular do espalhamento quasielástico ${ }^{7} \mathrm{Be}+{ }^{9} \mathrm{Be}$ para $\mathrm{E}_{\text {lab }}=23,1 \mathrm{MeV}$ comparada com os cálculos realizados com o formalismo de canais acoplados.

Pode-se notar que os canais inelásticos aparentemente têm pouca contribuição no espalhamento. 
Os valores das profundidades dos potenciais foram variados através de uma busca automática, de tal forma a minimizar os valores de $\chi^{2} / N$. Os parâmetros do potencial $\left({ }^{3} \mathrm{He}+{ }^{9} \mathrm{Be}\right)_{I I}$ foram desconsiderados devido ao fato de terem a mesma geometria dos parâmetros do potencial $\left({ }^{3} \mathrm{He}+{ }^{9} \mathrm{Be}\right)_{I}$. Os parâmetros dos potenciais obtidos estão apresentados na Tabela 5.7, os resultados obtidos podem ser vistos na Fig. 5.8.

Tabela 5.7: Parâmetros dos potenciais obtidos através de uma busca automática no cálculo do espalhamento quasielástico através do formalismo de canais acoplados.

\begin{tabular}{ccccccccccc}
\hline Sistema & $\begin{array}{c}V_{0} \\
(\mathrm{MeV})\end{array}$ & $\begin{array}{c}r_{0} \\
(\mathrm{fm})\end{array}$ & $\begin{array}{c}a_{0} \\
(\mathrm{fm})\end{array}$ & $\begin{array}{c}W_{i} \\
(\mathrm{MeV})\end{array}$ & $\begin{array}{c}r_{i} \\
(\mathrm{fm})\end{array}$ & $\begin{array}{c}a_{i} \\
(\mathrm{fm})\end{array}$ & $\begin{array}{c}V_{S O} \\
(\mathrm{MeV})\end{array}$ & $\begin{array}{c}r_{S O} \\
(\mathrm{fm})\end{array}$ & $\begin{array}{c}a_{S O} \\
(\mathrm{fm})\end{array}$ & $\chi^{2} / N$ \\
\hline${ }^{4} \mathrm{He}+{ }^{3} \mathrm{He}$ & $80,76(1)$ & 2,52 & - & - & - & - & $3,81(1)$ & 2,52 & - & \\
${ }^{4} \mathrm{He}+{ }^{9} \mathrm{Be} a$ & $140,64(1)$ & 1,60 & 0,64 & $49,41(1)$ & 1,60 & 0,64 & - & - & - & 12,1 \\
${ }^{3} \mathrm{He}+{ }^{9} \mathrm{Be}$ & $55,32(1)$ & 1,85 & 0,58 & $49,59(1)$ & 1,85 & 0,58 & $2,65(1)$ & 1,85 & 0,58 & \\
\hline${ }^{4} \mathrm{He}+{ }^{3} \mathrm{He}$ & $83,39(1)$ & 2,52 & - & - & - & - & $3,97(1)$ & 2,52 & - & \\
${ }^{4} \mathrm{He}+{ }^{9} \mathrm{Be} b$ & $199,57(1)$ & 1,20 & 0,51 & $199,97(1)$ & 1,20 & 1,99 & $5,63(1)$ & 1,20 & 0,51 & 19,6 \\
${ }^{3} \mathrm{He}+{ }^{9} \mathrm{Be}$ & $99,99(1)$ & 1,85 & 0,58 & $2,09(1)$ & 1,85 & 0,58 & $2,45(1)$ & 1,85 & 0,58 & \\
\hline${ }^{4} \mathrm{He}+{ }^{3} \mathrm{He}$ & $87,59(1)$ & 2,52 & - & - & - & - & $3,57(1)$ & 2,52 & - & \\
${ }^{4} \mathrm{He}+{ }^{9} \mathrm{Be} c$ & $78,02(1)$ & 1,16 & 0,76 & $49,93(1)$ & 1,54 & 0,99 & - & - & - & 6,2 \\
${ }^{3} \mathrm{He}+{ }^{9} \mathrm{Be}$ & $20,34(1)$ & 1,85 & 0,58 & $1,13(1)$ & 1,85 & 0,58 & $2,36(1)$ & 1,85 & 0,58 & \\
\hline
\end{tabular}

Nota-se um grande aumento nas profundidades imaginárias de alguns dos potenciais de interação dos fragmentos com o alvo, principalmente para ${ }^{4} \mathrm{He}+{ }^{9} \mathrm{Be}$. Isso ocorre pois, para esse sistema de cluster, essa interação apresenta forte contribuição para a perda de fluxo do canal elástico.

Desta forma, verifica-se que os canais inelásticos possuem pouca contribuição no espalhamento e, portanto, o tratamento apenas com modelo óptico mostra-se suficiente para a descrição da distribuição quasielástica ${ }^{7} \mathrm{Be}+{ }^{9} \mathrm{Be}$ desse experimento. 

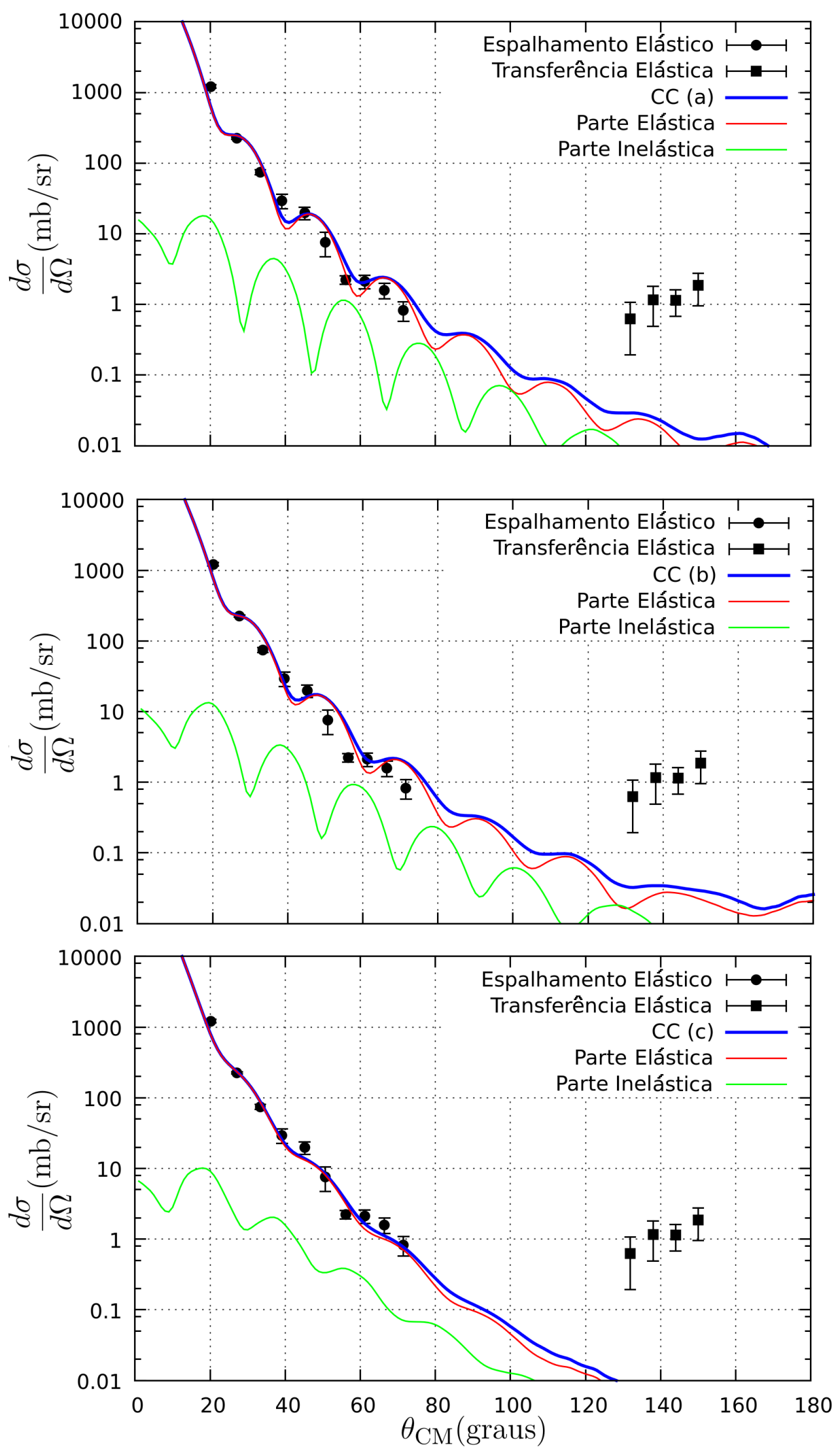

Figura 5.8: Distribuição angular do espalhamento quasielástico ${ }^{7} \mathrm{Be}+{ }^{9} \mathrm{Be}$ para $\mathrm{E}_{\text {lab }}=23,1 \mathrm{MeV}$ comparada com os cálculos realizados com o formalismo de canais acoplados variando as profundidades real e imaginária dos potenciais. 


\subsection{Cálculos de DWBA}

As reações de transferência são importantes, pois podem fornecer informações sobre a normalização da função de onda do estado ligado (nesse caso ${ }^{7} \mathrm{Be}+2 n$ ), que está relacionada à probabilidade de se encontrar o núcleo nesse estado. A reação de transferência estudada $\left({ }^{9} \mathrm{Be}\left({ }^{7} \mathrm{Be},{ }^{9} \mathrm{Be}\right){ }^{7} \mathrm{Be}\right)$ é do tipo pick-up, ou seja, o projétil recebe núcleons do alvo. Essa reação tem $Q=0$, pois as massas iniciais e finais são as mesmas.

Nesse sistema, a detecção de um ${ }^{9} \mathrm{Be}$ em ângulos dianteiros representa um ${ }^{7} \mathrm{Be}$ em ângulos traseiros, entretanto, o ${ }^{9}$ Be pode ser uma partícula de recuo proveniente do espalhamento elástico ou o projétil após uma reação de transferência. Utilizou-se o potencial Woods-Saxon (WS(l)), obtido através do modelo óptico, como ponto de partida. Como não é possível separar a contribuição do canal de transferência da contribuição do canal elástico, torna-se necessário acrescentar esse espalhamento nos cálculos, realizados, por sua vez, através de cálculos de DWBA. Para isso, o ${ }^{9}$ Be foi considerado como sendo ${ }^{7} \mathrm{Be}+2 n$. Para a interação ${ }^{7} \mathrm{Be}+{ }^{9} \mathrm{Be}$ são necessários os potenciais referentes as interações ${ }^{7} \mathrm{Be}+{ }^{9} \mathrm{Be},{ }^{7} \mathrm{Be}+{ }^{7} \mathrm{Be}$ e ${ }^{7} \mathrm{Be}+2 n$ (sendo esse um estado ligado, descrito apenas pela parte real do potencial óptico). Para o sistema ${ }^{7} \mathrm{Be}+{ }^{7} \mathrm{Be}$ foi utilizado o potencial do sistema similar ${ }^{7} \mathrm{Li}+{ }^{7} \mathrm{Li}$ encontrado na literatura [46]. Para ${ }^{7} \mathrm{Be}+2 n$ foram usados valores padronizados para a difusividade $\left(a_{0}=0,65 \mathrm{fm}\right)$ e raio $\left(r_{0}=1,25 \mathrm{fm}\right)$ e a profundidade $(V)$ foi variada automaticamente para reproduzir a energia de ligação do estado $\left(\mathrm{E}_{\mathrm{x}}=20,563 \mathrm{MeV}\right)$. Os cálculos foram realizados para a transferência simultânea de dois nêutrons. A Tabela 5.8 mostra os potenciais usados neste cálculo.

Tabela 5.8: Potenciais usados para os cálculos de DWBA.

\begin{tabular}{|c|c|c|c|c|c|c|c|c|}
\hline Sistema & $\begin{array}{l}\text { Sistema } \\
\text { Original }\end{array}$ & $\begin{array}{c}V \\
(\mathrm{MeV})\end{array}$ & $\begin{array}{c}r_{0} \\
(\mathrm{fm})\end{array}$ & $\begin{array}{c}a_{0} \\
(\mathrm{fm})\end{array}$ & $\begin{array}{c}W \\
(\mathrm{MeV})\end{array}$ & $\begin{array}{c}r_{i} \\
(\mathrm{fm})\end{array}$ & $\begin{array}{c}a_{i} \\
(\mathrm{fm})\end{array}$ & Ref. \\
\hline${ }^{7} \mathrm{Be}+{ }^{7} \mathrm{Be}$ & ${ }^{7} \mathrm{Li}+{ }^{7} \mathrm{Li}$ & 107,8 & 0,75 & 0,85 & 37,9 & 0,91 & 0,757 & {$[46]$} \\
\hline${ }^{7} \mathrm{Be}+2 n$ & - & variável & 1,25 & 0,65 & - & - & - & \\
\hline
\end{tabular}


A amplitude espectroscópica está relacionada com a normalização da função de onda do estado ligado, e pode ser obtida através desses cálculos, variando-a para melhor reproduzir os dados experimentais. Para esses cálculos foi considerado que os dois nêutrons de valência do ${ }^{9}$ Be estão na camada $1 p_{3 / 2}$ e são transferidos para essa mesma camada núcleo de ${ }^{7} \mathrm{Be}$.

Primeiramente, a amplitude espectroscópica foi variada entre 0 a 1, com passo de 0,1 , de forma a se obter um valor de referência. O resultado obtido pode ser visto na Fig. 5.9.

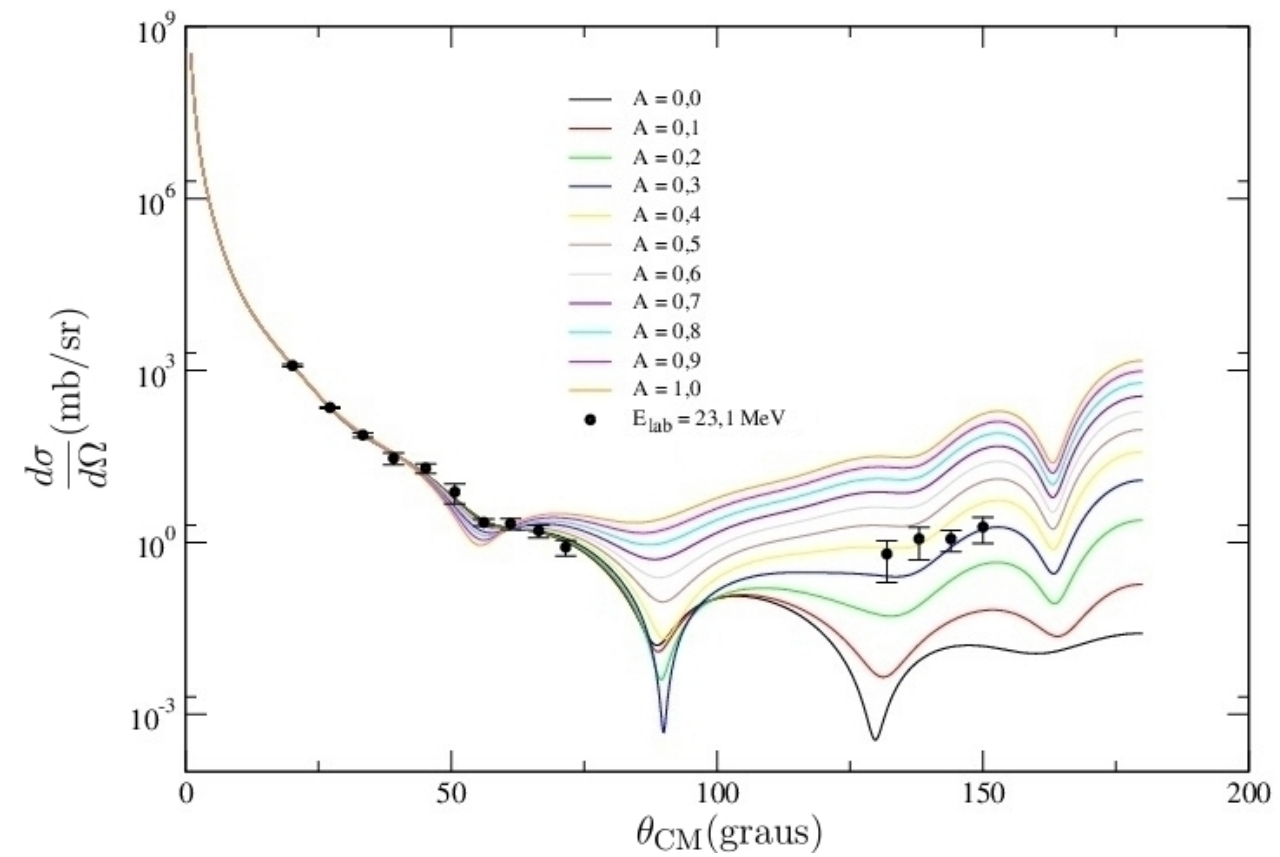

Figura 5.9: Distribuição angular do espalhamento quasielástico ${ }^{7} \mathrm{Be}+{ }^{9} \mathrm{Be}$ para $\mathrm{E}_{\text {lab }}=23,1 \mathrm{MeV}$ comparada com os cálculos de DWBA variando a amplitude espectroscópica $A$. Para a parte elástica foi utilizado um potencial do tipo Woods-Saxon (l) obtido nesse trabalho.

Observando os resultados apresentados na Fig. 5.9, pode-se notar que o valor da amplitude espectroscópica que melhor reproduz os dados está entre 0,3 e 0,4. A partir disso, foi feita uma busca manual, primeiramente passando a curva pelos dois primeiros pontos experimentais referentes a transferência elástica (Conj. I) e, em seguida, pelos dois últimos (Conj. II), de forma que fosse possível estimar a média e incerteza. A Tabela 5.9 mostra os valores obtidos com esse método. 
Tabela 5.9: Amplitudes espectroscópicas obtidas com o potencial Woods-Saxon $(\mathrm{WS}(\mathrm{l}))$.

\begin{tabular}{ccc}
\hline Conj. & $A$ & $\chi^{2} / N$ \\
\hline I & 0,41 & 2,001 \\
II & 0,32 & 0,546 \\
\hline Média & $0,36(5)$ & 0,804 \\
\hline
\end{tabular}

Através dessa busca foi obtido o valor médio para a amplitude espectroscópica de $A=0,36(5)$, que fornece o fator espectroscópico $S F=0,13(4)$, de acordo com a Eq. (4.43). A Fig. 5.10 mostra o resultado obtido.

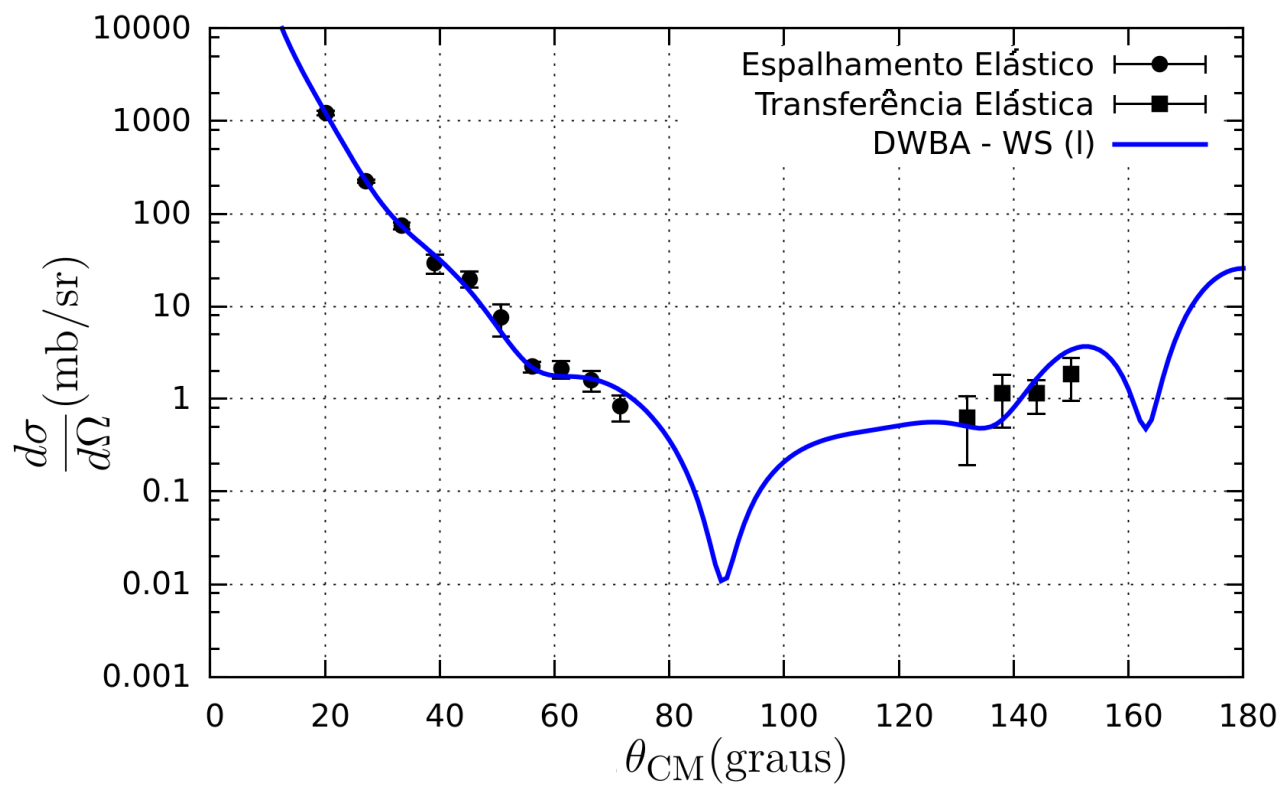

Figura 5.10: Distribuição angular do espalhamento quasielástico ${ }^{7} \mathrm{Be}+{ }^{9} \mathrm{Be}$ para $\mathrm{E}_{\text {lab }}=23,1 \mathrm{MeV}$ comparada com cálculos de DWBA variando a amplitude espectroscópica $A$. Foi obtido o resultado $A=0,36(5)$ e $S F=0,13(4)$.

A partir do potencial misto (SPP+WS) obtido neste trabalho (ver Fig. 5.6) e utilizando a metodologia já apresentada para estimar a amplitude espectroscópica, foi obtido o resultado apresentado na Fig. 5.11.

Observando os resultados apresentados na Fig. 5.11, pode-se notar que para este potencial a amplitude espectroscópica que reproduz os primeiros três pontos 


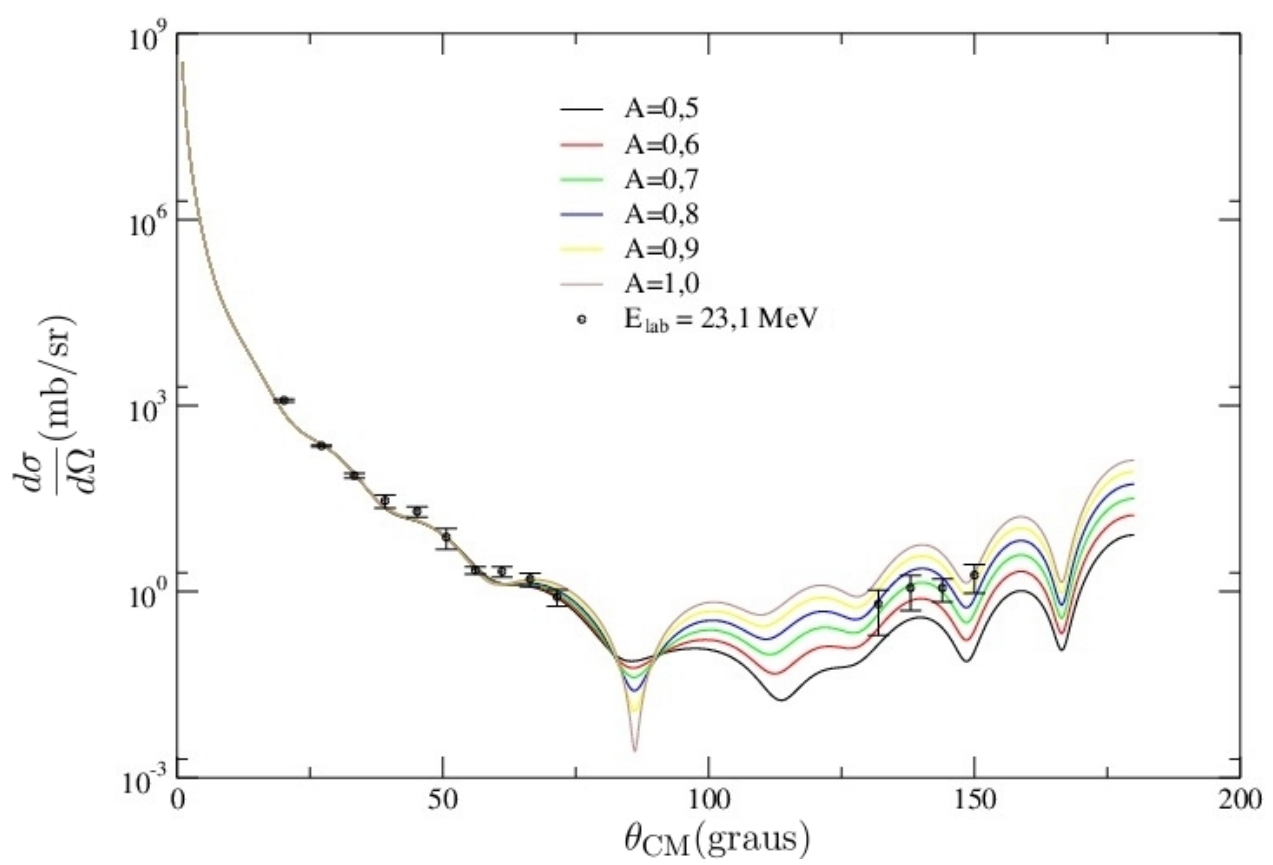

Figura 5.11: Distribuição angular do espalhamento quasielástico ${ }^{7} \mathrm{Be}+{ }^{9} \mathrm{Be}$ para $\mathrm{E}_{\text {lab }}=23,1 \mathrm{MeV}$ comparada com os cálculos de DWBA variando a amplitude espectroscópica $A$. Para a parte elástica foi utilizado um potencial do tipo São Paulo para a parte real e Woods-Saxon para a parte imaginária.

referentes a transferência elástica é $A=0,7$ (Conj. III), enquanto que o valor que reproduz o último ponto é $A=0,9$ (Conj. IV). Portanto, foi feita uma média ponderada entre esses valores, utilizando o número de pontos como peso, de forma que fosse possível estimar a média e a incerteza desses valores. A Tabela 5.10 mostra os valores obtidos com esse método.

Tabela 5.10: Amplitudes espectroscópicas obtidas com o potencial São Paulo na parte real e Woods-Saxon na parte imaginária.

\begin{tabular}{cccc}
\hline Conj. & $A$ & Peso & $\chi^{2} / N$ \\
\hline III & 0,7 & 3 & 4,3 \\
IV & 0,9 & 1 & 4,5 \\
\hline Média & $0,7(1)$ & & 4,2 \\
\hline
\end{tabular}

Analogamente ao processo realizado anteriormente, o valor obtido para a amplitude espectroscópica é de $A=0,7(1)$, fornecendo o fator espectroscópico de $S F=$ 
0,5(1). A Fig. 5.12 mostra o resultado obtido.

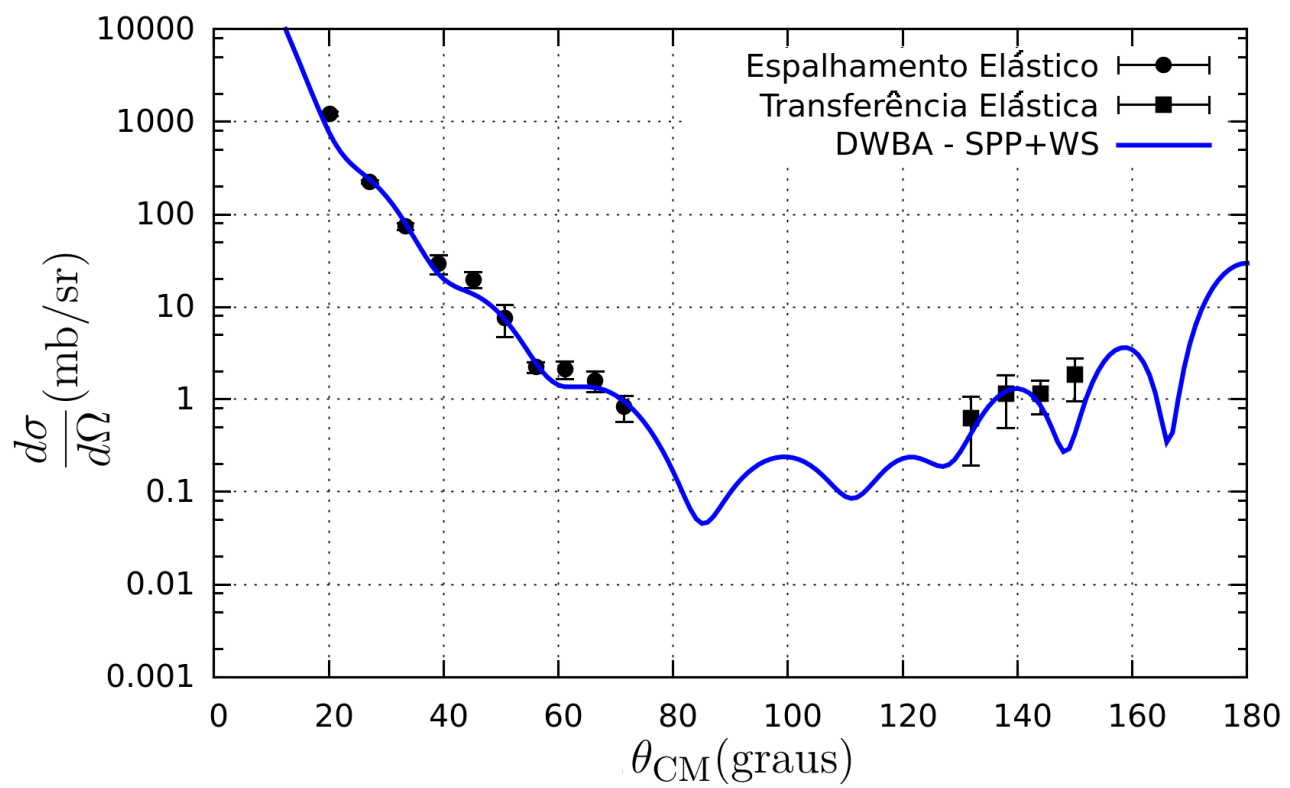

Figura 5.12: Distribuição angular do espalhamento quasielástico ${ }^{7} \mathrm{Be}+{ }^{9} \mathrm{Be}$ para $\mathrm{E}_{\mathrm{lab}}=23,1 \mathrm{MeV}$ comparada com os cálculos de DWBA variando a amplitude espectroscópica $A$. Foi obtido o resultado $A=0,7(1)$ e $S F=0,5(1)$.

Observando os dois resultados obtidos para o fator espetroscópico, pode-se notar que a configuração de cluster $\left({ }^{7} \mathrm{Be}+2 n\right)$ para o ${ }^{9} \mathrm{Be}$ tem, em ambos os casos, baixa probabilidade de ser detectado. Como a função de onda do estado ligado é uma superposição dos possíveis estados, sendo o favorável a esse tipo de transferência dupla esse estado ligado de baixa probabilidade, essa reação de pick-up se mostra difícil de ser detectada. Devido ao valor de energia utilizado neste trabalho $\left(\mathrm{E}_{\mathrm{CM}}=13\right.$ $\mathrm{MeV}$ ), essa reação de transferência se torna ainda mais improvável devido a alta energia de ligação da configuração de cluster. Esses fatores corroboram com a baixa quantidade de contagens obtidas para essa reação, além do fato de ser uma reação inédita, sem informações na literatura.

Porém, através de dois potenciais distintos, foram obtidos dois valores diferentes para o fator espectroscópico. Um teste simples de compatibilidade pode ser feito através da expressão [47]: 


$$
\frac{\left|S F_{1}-S F_{2}\right|}{\sqrt{\delta_{1}^{2}+\delta_{2}^{2}}}>3
$$

onde $\delta$ são as incertezas relativas aos fatores espectroscópicos obtidos. Essa expressão se baseia no princípio de que em uma distribuição gaussiana 99,73\% dos dados estão em uma região de até $3 \delta$ da média. Através desse teste, observa-se que os dois valores se encontram a aproximadamente 2,6 incertezas um do outro, mostrando que são dados compatíveis. A Fig. 5.13 mostra a comparação dos espectros obtidos:

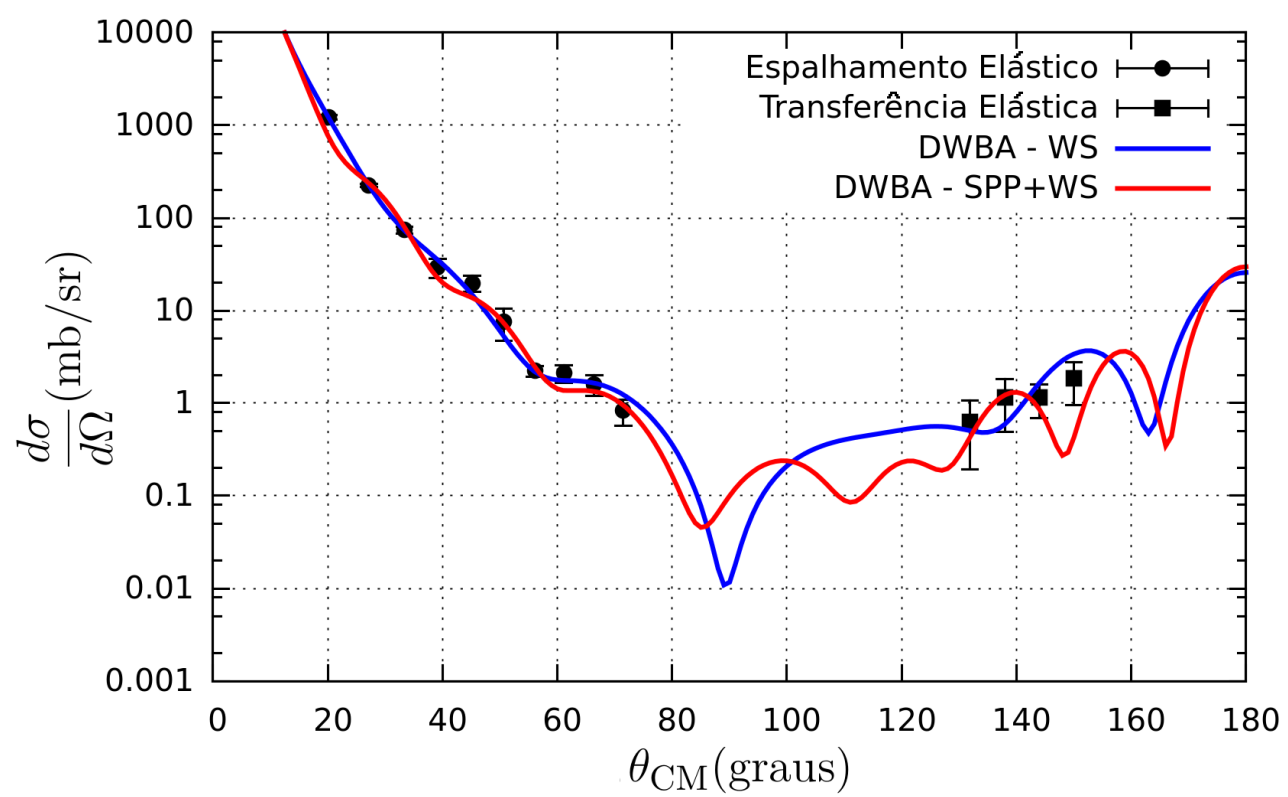

Figura 5.13: Distribuição angular do espalhamento quasielástico ${ }^{7} \mathrm{Be}+{ }^{9} \mathrm{Be}$ para $\mathrm{E}_{\text {lab }}=23,1 \mathrm{MeV}$ comparada com os cálculos de DWBA com as duas amplitudes espectroscópica obtidas neste trabalho $A_{1}=0,36(5)$ e $A_{2}=0,7(1)$.

Observando as Figs. 5.3 e 5.6, onde são apresentados os resultados dos cálculos de modelo óptico, observa-se que a seção de choque obtida em ângulos traseiros é baixa se comparada com a transferência elástica. Entretanto, os potenciais elásticos têm grande influência na seção de choque onde deveria predominar a influência da transferência elástica. Isso indica que nessa região angular as partículas espalhadas têm grande sensibilidade às regiões mais internas dos potenciais elásticos.

A boa concordância obtida entre os dados experimentais e os cálculos de DWBA 
(que utiliza potenciais previamente obtidos para o espalhamento quasielástico), dão confiabilidade aos dados e cálculos obtidos neste trabalho.

\subsection{Seção de Choque de Reação}

Um método extremamente simples de se comparar os potenciais obtidos é comparando a seção de choque de reação, que consiste na soma de todos os processos não elásticos, indicando a perda de fluxo do canal elástico. A seção de choque de reação pode ser obtida pela expressão [30]:

$$
\sigma_{\text {reac }}=\frac{\pi}{k^{2}} \sum_{l}(2 L+1)\left(1-\left|S_{l}\right|^{2}\right)
$$

onde $S_{l}$ corresponde aos elementos diagonais da matriz de espalhamento elástico. A Tabela 5.11 mostra os valores obtidos com os cálculos realizados nesse trabalho.

Tabela 5.11: Seções de choque de reação dos potenciais que melhor reproduzem os dados experimentais do sistema ${ }^{7} \mathrm{Be}+{ }^{9} \mathrm{Be}$ para $\mathrm{E}_{\mathrm{lab}}=23,1 \mathrm{MeV}$.

\begin{tabular}{ccccc}
\hline Cálculo & Índice & $\begin{array}{c}\sigma_{\text {elást }} \\
(\mathrm{mb})\end{array}$ & $\begin{array}{c}\sigma_{\text {inelást }} \\
(\mathrm{mb})\end{array}$ & $\begin{array}{c}\sigma_{\text {total }} \\
(\mathrm{mb})\end{array}$ \\
\hline MO - WS & $(\mathrm{k})$ & 1172,2 & & 1172,2 \\
MO - WS & $(\mathrm{l})$ & 1169,9 & & 1169,9 \\
\hline MO - SPP & $(\mathrm{d})$ & 823,8 & & 823,8 \\
MO - SPP & $(\mathrm{e})$ & 1495,6 & & 1495,6 \\
\hline MO - (SPP + WS $)$ & - & 1895,5 & & 1895,5 \\
\hline CC & $(\mathrm{a})$ & 1524,3 & 11,3 & 1535,6 \\
CC & $(\mathrm{b})$ & 1466,14 & 9,4 & 1475,5 \\
CC & $(\mathrm{c})$ & 1740,1 & 6,7 & 1746,9 \\
\hline DWBA - WS & - & - & - & 1165,5 \\
DWBA - SPP+WS & - & - & - & 1889,3 \\
\hline Média & & $1383(331)$ & $9,1(22)$ & $1437(351)$ \\
\hline
\end{tabular}

Com exceção do $\operatorname{SPP}(d)$ que, conforme já foi discutido, não parece ser um bom 
modelo para reproduzir esse sistema devido às deformidades na densidade nuclear, pode-se notar que as seções de choque de reação para diferentes cálculos são relativamente próximas entre si, flutuando ao redor da média de $1437 \mathrm{mb}$. A Tabela 5.12 apresenta os valores de seção de choque de reação obtidos da literatura para o sistema ${ }^{7} \mathrm{Be}+{ }^{9} \mathrm{Be}$, utilizando diferentes cálculos $[2,1,3,41]$.

Tabela 5.12: Valores médios para as seções de choque de reações para potenciais encontrados na literatura.

\begin{tabular}{ccccc}
\hline Sistema & Cálculo & $\begin{array}{c}\text { Energia } \\
(\mathrm{MeV})\end{array}$ & $\begin{array}{c}\sigma_{\text {total }} \\
(\mathrm{mb})\end{array}$ & Ref. \\
\hline${ }^{7} \mathrm{Be}+{ }^{9} \mathrm{Be}$ & $\mathrm{MO}$ & 23,7 & 1295 & {$[2]$} \\
${ }^{7} \mathrm{Be}+{ }^{9} \mathrm{Be}$ & $\mathrm{CC}$ & 23,7 & 1392 & {$[2]$} \\
${ }^{7} \mathrm{Be}+{ }^{9} \mathrm{Be}$ & $\mathrm{CDCC}$ & 23,7 & 1363 & {$[2]$} \\
${ }^{7} \mathrm{Be}+{ }^{9} \mathrm{Be}$ & $\mathrm{MO}$ & 17,0 & 1060 & {$[1]$} \\
${ }^{7} \mathrm{Be}+{ }^{9} \mathrm{Be}$ & $\mathrm{MO}$ & 19,0 & 1116 & {$[1]$} \\
${ }^{7} \mathrm{Be}+{ }^{9} \mathrm{Be}$ & $\mathrm{MO}$ & 21,0 & 1197 & {$[1]$} \\
${ }^{7} \mathrm{Li}+{ }^{9} \mathrm{Be}$ & $\mathrm{MO}$ & 34,0 & 1524 & {$[3]$} \\
${ }^{7} \mathrm{Li}+{ }^{9} \mathrm{Be}$ & $\mathrm{MO}$ & 24,0 & 1415 & {$[41]$} \\
\hline
\end{tabular}

Comparando o valor obtido nesse trabalho com dados para os sistemas ${ }^{7} \mathrm{Be}+{ }^{9} \mathrm{Be}$ e ${ }^{7} \mathrm{Li}+{ }^{9} \mathrm{Be}$ obtidos da literatura, percebe-se que estão bastante próximos, mostrando que as medidas e cálculos aqui realizados apresentam bom acordo com os dados da literatura. Isso mostra que os métodos experimentais utilizados neste trabalho, assim como os resultados obtidos, podem ser considerados bastante confiáveis. 


\section{Capítulo 6}

\section{Conclusões}

Foram realizadas medidas de espalhamento para o sistema ${ }^{7} \mathrm{Be}+{ }^{9} \mathrm{Be}$ na energia de $E_{\mathrm{CM}}=13 \mathrm{MeV}\left(E_{\mathrm{lab}}=23,1 \mathrm{MeV}\right)$. O experimento foi realizado utilizando um feixe primário de ${ }^{6} \mathrm{Li}$, produzido pelo acelerador Pelletron, e o sistema RIBRAS para seleção e focalização do feixe radioativo. Foi utilizada a reação $\mathrm{LiF}\left({ }^{6} \mathrm{Li},{ }^{7} \mathrm{Be}\right)$ como reação de produção no alvo primário. Como alvo secundário foram utilizados ${ }^{9} \mathrm{Be} e$ ${ }^{197} \mathrm{Au}$, este último para a normalização dos dados. A calibração dos detectores foi feita para confirmar a identificação de partículas, além de proporcionar a conversão entre canais e energia, tornando possível a identificação dos picos com menor número de contagens. Isso possibilitou a confirmação do canal de transferência elástica, utilizado para complementar a distribuição elástica na região de ângulos traseiros.

A análise teórica foi realizada, primeiramente, utilizando o formalismo de Modelo Óptico, empregando fatores de forma do tipo Woods- Saxon, além do potencial de São Paulo. Com esses resultados nota-se que diversos potenciais Woods-Saxon reproduzem relativamente bem os dados experimentais, porém aqueles que apresentam potenciais imaginários rasos e de longo alcance $\left(r_{i}\right.$ e/ou $a_{i}$ grandes) descrevem melhor as oscilações presentes nos dados experimentais.

Cálculos realizados utilizando o potencial de São Paulo mostram que os resultados descrevem bem os dados experimentais. Algumas oscilações previstas pelos cálculos não são vistas nos dados experimentais, isso se deve a grande resolução angular do experimento, que atenua a amplitude das oscilações. Porém, o melhor resultado foi obtido utilizando valores de normalização não realistas (muito diferen- 
tes do que o previsto pelo modelo).

Os formatos dos potenciais obtidos, de curto alcance na parte real e rasos e de longo alcance na parte imaginária, foram um indicativo de que os dados poderiam ser representados por um potencial misto, São Paulo para a parte real juntamente com Woods-Saxon para a parte imaginária. Através de uma variação automática dos parâmetros do potencial Woods-Saxon, obteve-se uma boa reprodução dos dados experimentais, confirmando que o potencial de interação tem componente imaginária de longo alcance.

Foram realizados cálculos de canais acoplados para estimar a contribuição do primeiro estado excitado do ${ }^{7}$ Be no canal elástico, visto que estes não podem ser separados devido à baixa energia de excitação e à resolução em energia do experimento. Os resultados destes cálculos indicam que a contribuição inelástica de primeira ordem é de baixa intensidade, podendo representar, então, o espalhamento pelo modelo óptico.

Finalmente, através de cálculos de DWBA obteve-se a contribuição da reação de dupla transferência de nêutrons na distribuição elástica, com isso pôde-se estimar a normalização da função de onda para o estado ligado do ${ }^{9} \mathrm{Be}$, bem como a probabilidade desse núcleo ser encontrado nas configurações de estado ligado ${ }^{7} \mathrm{Be}+2 n$ com momentos angulares fixos. Porém, utilizando dois potenciais elásticos diferentes foram obtidos dois valores diferentes, mas compatíveis em 2,6 incertezas, para o fator espectroscópico, indicando que nessa região angular a estrutura interna dos potenciais nucleares tem grande influência na seção de choque. Esse tipo de medida não foi encontrada na literatura, portanto assumiu-se que foi uma medida e cálculos inéditos. 


\section{Referências Bibliográficas}

[1] S. Verma, J. J. Das, A. Jhingan, K. Kalita, S. Barua, K. S. Golda, N. Madhavan, P. Sugathan, S. Nath, T. Varughese, J. Gehlot, S. Mandal, S. Ranjit, P. K. Sahu, B. John, B. K. Nayak, A. Saxena, S. K. Datta, e R. Singh. Measurements of elastic scattering for ${ }^{7} \mathrm{Be},{ }^{7} \mathrm{Li}+{ }^{9} \mathrm{Be}$ systems e fusion cross sections for ${ }^{7} \mathrm{Li}+{ }^{9} \mathrm{Be}$ system. Eur. Phys. J. Spec. Top., 150(1):75-78, nov 2007.

[2] K. C. C. Pires. Estudo de Sistemas de Núcleos Exóticos Leves ${ }^{6} \mathrm{He},{ }^{7} \mathrm{Be}+{ }^{9} \mathrm{Be}$. Tese de Doutorado, Universidade de São Paulo, 2011.

[3] K. A. Weber, K. Meier-Ewert, H. Schmidt-Böcking, e K. Bethge. Elastic scattering of ${ }^{7} \mathrm{Li}$ from light target nuclei. Nucl. Phys. A, 186(1):145-151, may 1972.

[4] M. A. G. Alvarez, L. C. Chamon, M. S. Hussein, D. Pereira, L. R. Gasques, E. S. Rossi, e C. P. Silva. A parameter-free optical potential for the heavy-ion elastic scattering process. Nucl. Phys. A, 723(1-2):93-103, jul 2003.

[5] P. N. Faria. Estudo do Espalhamento Elástico e Reações de Feixes Secundários de Núcleos Exóticos. Tese de Doutorado, Universidade de São Paulo, 2008.

[6] R. Lichtenthaler, A. Lépine-Szily, A. C. C. Villari, W. Mittig, V. J. G. Porto, e C. V. Acquadro. Effect of the $\alpha$-transfer reaction on the elastic scattering of ${ }^{12} \mathrm{C}+{ }^{24} \mathrm{Mg}$. Phys. Rev. C, 26(6):2487-2495, dec 1982.

[7] R. Z. Denke. Fator $S$ Astrofísico para a reação de Captura ${ }^{4} \mathrm{He}(t, \gamma)^{7} \mathrm{Li}$ pela investigação da reação de transferência elástica no sistema ${ }^{7} \mathrm{Li}+{ }^{4} \mathrm{He}$. Tese de Doutorado, Universidade de São Paulo, 2007. 
[8] O. C. Junior. Fator-S Astrofísico da reação de captura de próton ${ }^{8} \mathrm{Li}(p, \gamma)^{9} \mathrm{Be}$ através do estudo da reação de transferência elástica ${ }^{9} \mathrm{Be}\left({ }^{8} \mathrm{Li},{ }^{9} \mathrm{Be}\right)$. Dissertação de Mestrado, Universidade de São Paulo, 2009.

[9] M. C. Morais. Efeito de Estados de Estrutura Alfa no Espalhamento ${ }^{16} \mathrm{O}+{ }^{12} \mathrm{C} e$ na Reação de captura ${ }^{12} C(\alpha, \gamma)^{16} O$ de Interesse Astrofísico. Tese de Doutorado, Universidade de São Paulo, 2010.

[10] G. R. Satchler. Introduction to Nuclear Reactions. The MACMILLAN PRESS LTD, 1990.

[11] L. Mu Young. Twinsol: A dual superconducting solenoid ion-optical system for the production e study of low-energy radioactive nuclear beam reactions. Tese de Doutorado, University of Michigan, 2002.

[12] NNDC. Chart of Nuclides, http://www.nndc.bnl.gov/chart/, Data de Acesso $26 / 08 / 2016$.

[13] R. Lichtenthäler, A. Lépine-Szily, V. Guimar aes, C. Perego, V. Placco, O. Camargo, R. Denke, P. N. de Faria, E. A. Benjamim, N. Added, G. F. Lima, M. S. Hussein, J. Kolata, e A. Arazi. Radioactive Ion beams in Brazil (RIBRAS). Eur. Phys. J. A, 25(S1):733-736, sep 2005.

[14] A. Lépine-Szily, R. Lichtenthäler, e V. Guimarães. The Radioactive Ion Beams in Brazil (RIBRAS) facility. Eur. Phys. J. A, 50(8):128, aug 2014.

[15] E. Chainsson e S. McMillan. Astronomy Today. Pearson, 2014.

[16] TUNL - Nuclear Data Evaluation. Energy Level Diagrams for $A=3-20$, http://www.tunl.duke.edu/nucldata, 18/02/2016

[17] Isotopes of Beryllium, http://www.periodictable.com/Isotopes/004.7/index.html, Data de Acesso 31/03/2016.

[18] National Electrostatics Corporation. Source of Negative Ions by Cesium Sputtering - SNICS II, http://www.pelletron.com/negion.htm, Data de Acesso $16 / 08 / 2015$. 
[19] O. Sala e G. Spalek. The Nuclear Structure Facility at the University of São Paulo. Nucl. Instruments Methods, 122:213-225, 1974.

[20] Departamento de Física Núclear do IFUSP. Acelerador Pelletron, http://portal.if.usp.br/fnc/pt-br/acelerador-pelletron, Data de Acesso 17/04/2016.

[21] National Electrostatics Corporation. Charging System, http://www.pelletron.com/charging.htm, Data de Acesso 16/08/2015.

[22] N. Ueta, V. H. Rotberg, D. Pereira, M. N. Rao, e O. Sala. Calibration Of The Tandem Pelletron Accelerator. Rev. Bras. Física, 6:413, 1976.

[23] R. Leo. Techniques for Nuclear e Particles Physics Experiments. SpringerVerlag, 1992.

[24] A. A. P. Suaide. Programas gráficos de aquisição e análise baseados no Root, http://www.dfn.if.usp.br/suaide, Data de Acesso 30/12/2014.

[25] Oak Ridge National Laboratory. Programas UPAK, http://www.phy.ornl.gov/, Data de Acesso 30/06/2014.

[26] N. Ueta, W. G. P. Engel, e J. J. G. Leandro. Confecção de Alvos Nucleares. 2007.

[27] M. H. Tabacniks. Os Elementos na Matéria. Tese de Livre Docência, Universidade de São Paulo, 2005.

[28] T. Williams e C. Kelley. Gnuplot, http://www.gnuplot.info/, Data de Acesso $20 / 04 / 2015$.

[29] R. Lichtenthäler. Código Fortran RIBRAS. Não Publicado.

[30] G. R. Satchler. Direct Nuclear Reactions. Oxford University Press, 1983.

[31] H. Feshbach, C.E. Porter e V.F. Weisskopf. Model for Nuclear Reactions with Neutrons. Phys. Rev., 96(2):448-464, oct 1954. 
[32] P. E. Hodgson. The nuclear optical model: Introductory Overview. Rep. Prog. Phys., 34:765-819, 1971.

[33] C. A. Bertulani. Introdução à Fúsica Nuclear. Editora UFRJ, Rio de Janeiro, 2007.

[34] R. D. Woods e D. S. Saxon. Diffuse Surface Optical Model for Nucleon-Nuclei Scattering. Phys. Rev., 95(2):577-578, jul 1954.

[35] L. C. Chamon, B. V. Carlson, L. R. Gasques, D. Pereira, C. De Conti, M. A. G. Alvarez, M. S. Hussein, M. A. Cândido Ribeiro, E. S. Rossi, e C. P. Silva. Toward a global description of the nucleus-nucleus interaction. Phys. Rev. C, 66(1):014610, jul 2002.

[36] L. C. Chamon. The São Paulo Potential. Nucl. Phys. A, 787(1-4):198-205, may 2007.

[37] P. A. M. Dirac. A new notation for quantum mechanics. Math. Proc. Cambridge Philos. Soc., 35(03):416, jul 1939.

[38] M. Born. Quantenmechanik der Stoßvorgänge. Zeitschrift für Phys., 38(1112):803-827, nov 1926.

[39] W. von Oertzen e H.G. Bohlen. Elastic transfer processes in heavy ion scattering. Phys. Rep., 19(1):1-61, jun 1975.

[40] I. J. Thompson. Coupled reaction channels calculations in nuclear physics. Comput. Phys. Reports, 7(4):167-212, apr 1988.

[41] K. W. Kemper, G. E. Moore, R. J. Puigh, e R. L. White. Spectroscopic information from the ${ }^{9} \mathrm{Be}\left({ }^{7} \mathrm{Li},{ }^{6} \mathrm{He}\right){ }^{10} \mathrm{~B}$ e ${ }^{9} \mathrm{Be}\left({ }^{7} \mathrm{Li},{ }^{6} \mathrm{Li}\right){ }^{10} \mathrm{Be}$ reactions. Phys. Rev. C, 15(5):1726-1731, may 1977.

[42] L. C. Chamon. SPOMC3 Computational Code. Não Publicado.

[43] B. Buck e A. C. Merchant. Cluster model of $A=7$ nuclei revisited, e the astrophysical S factors for ${ }^{3} \mathrm{He}(\alpha, \gamma){ }^{7} \mathrm{Be}$ e ${ }^{3} \mathrm{H}(\alpha, \gamma){ }^{7} \mathrm{Li}$ at zero energy. J. Phys. G Nucl. Phys., 14(10):L211, 1988. 
[44] N. C. Summers e F. M. Nunes. ${ }^{7}$ be breakup on heavy e light targets. Phys. Rev. C, (1):11602, jul.

[45] C. M. Perey e F. G. Perey. Compilation of phenomenological optical-model parameters 1954-1975. At. Data Nucl. Data Tables, 17(1):1-101, jan 1976.

[46] O. A. Momotyuk, N Keeley, K. W. Kemper, B. T. Roeder, A. M. Crisp, W. Cluff, B. G. Schmidt, M. Wiedeking, F. Maréchal, K. Rusek, S. Yu. Mezhevych, e J. Liendo. Target structure independent ${ }^{7} \mathrm{Li}$ elastic scattering at low momentum tranfers. Phys. Lett. B, 640(1-2):13-17, aug 2006.

[47] J. H. Vuolo. Fundamentos da Teoria de Erros. Ed. Edgard Blücher LTDA, 1993. 


\section{Apêndice A}

\section{Inputs FRESCO}

Nesse apêndice serão exemplificados os códigos utilizados para os cálculos com o programa FRESCO. Apenas os valores dos potenciais (partições) foram alterados para os cálculos. 


\section{A.1 Modelo Óptico - Woods-Saxon}

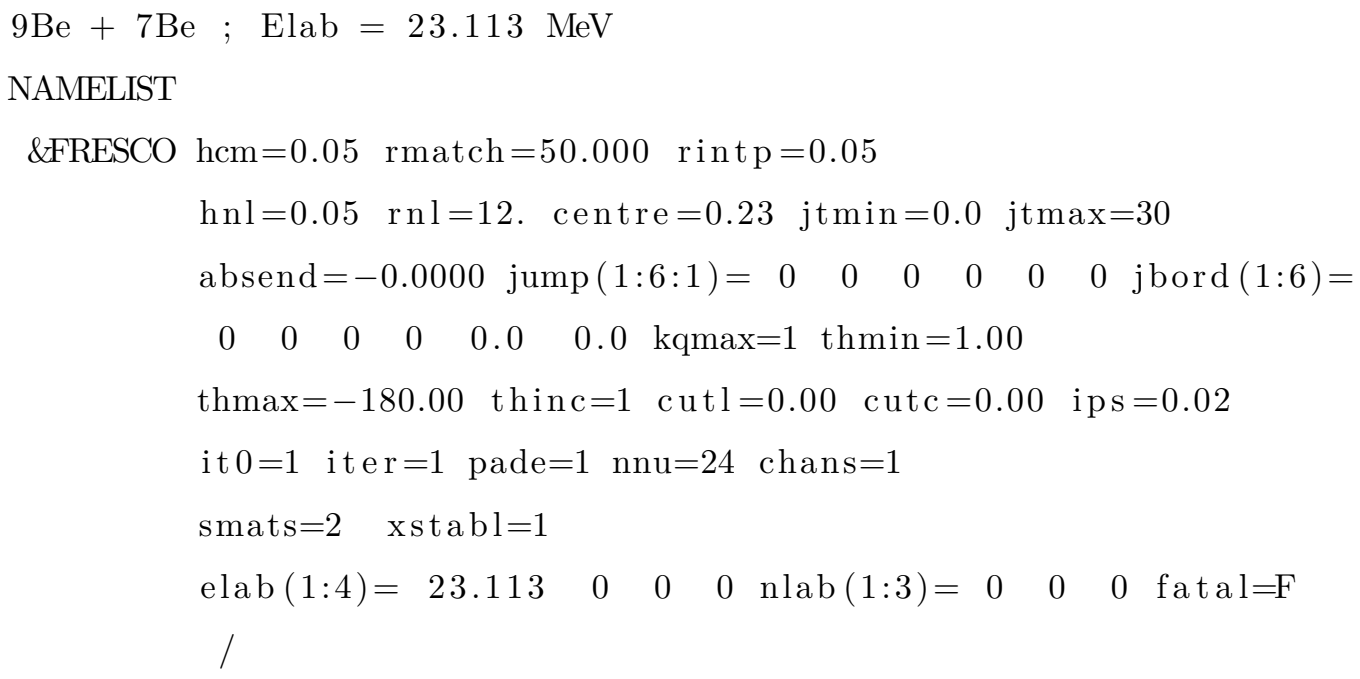




\section{A.2 Modelo Óptico - São Paulo}

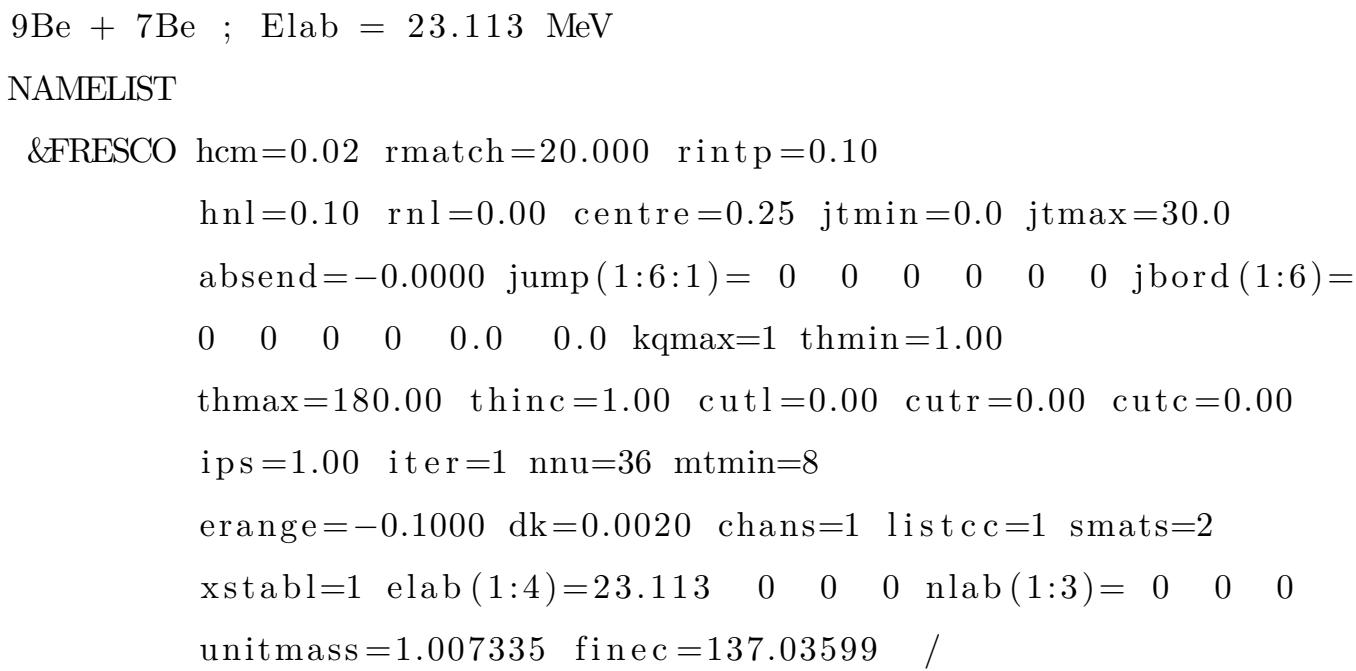




\section{A.3 Modelo Óptico - Misto}

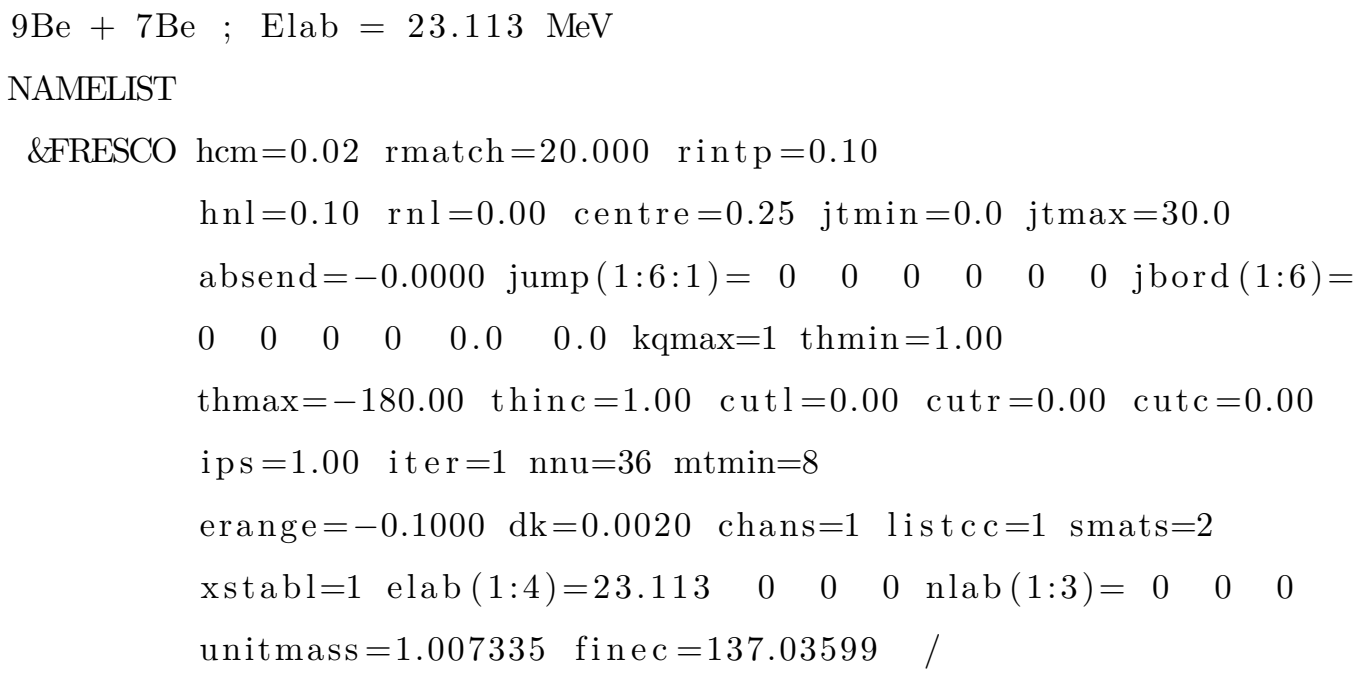




\section{A.4 Canais Acoplados}

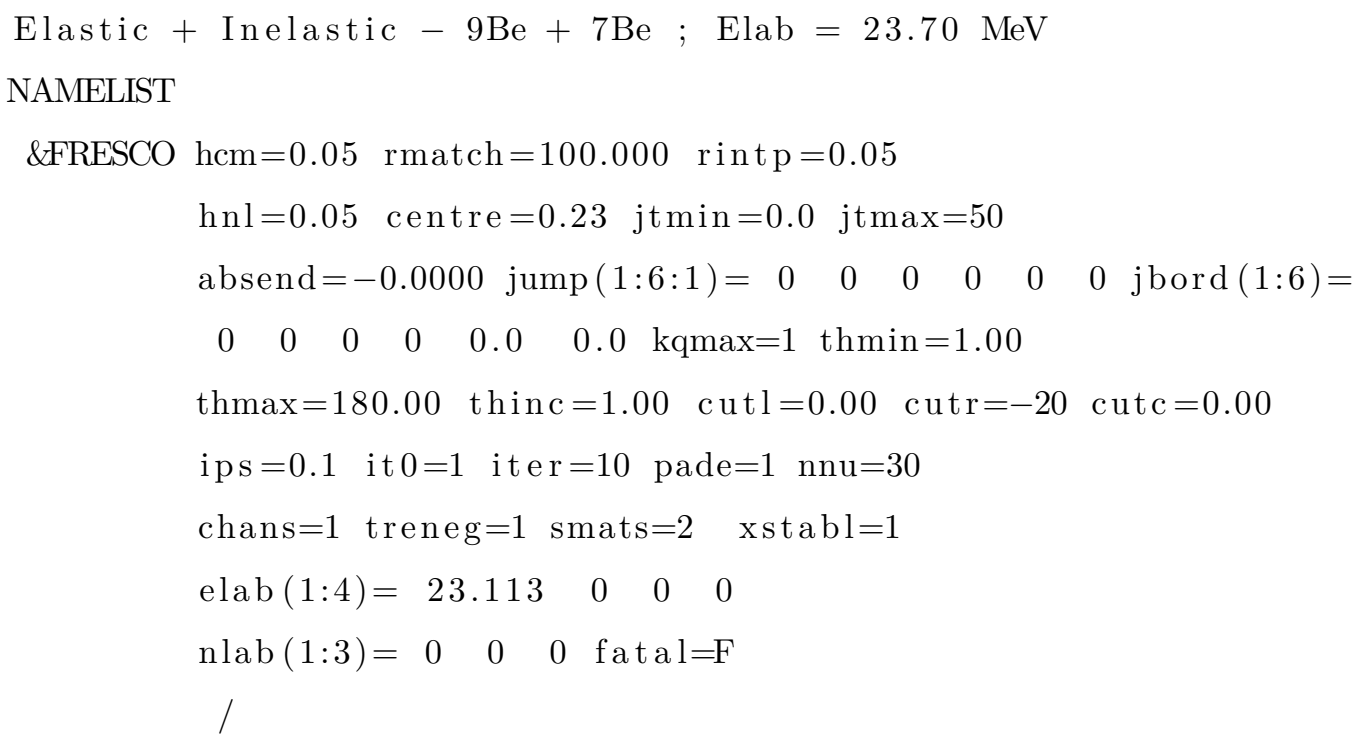

\&PARTITION namep='7Be' $\operatorname{massp}=7.016929 \quad \mathrm{zp}=4$.

namet $=' 9 B e '$ masst $=9.012182$ zt $=4 . \quad$ qval $=0.0 \quad$ pwf $=F \quad n e x=2 \quad /$

\&STATES $\mathrm{jp}=1.5$ bandp $=-1$ cpot $=1 \mathrm{jt}=1.5$

bandt $=-1 \quad /$

\&STATES jp $=0.5$ bandp $=-1$ ep $=0.4291$ cpot $=1$ jt $=0.0$

copyt=1 bandt $=-1 /$

$\&$ PARTITION namep='4He' $\operatorname{massp}=4.002603 \mathrm{zp}=2$.

namet $=' 12 \mathrm{C} '$ masst $=12.00000 \mathrm{zt}=6 . \quad \mathrm{qval}=24.692 \mathrm{pwf}=\mathrm{F}$

$\operatorname{nex}=1 \quad /$

\&STATES $\mathrm{jp}=0.0$ bandp $=1 \quad \mathrm{cpot}=3 \mathrm{jt}=0.0$

bandt $=1 \quad /$

\&partition /

$\&$ POT $\mathrm{kp}=1$ ap $=7.0$ at $=9.0 \quad \mathrm{rc}=1.20 \quad /$

\&POT $\mathrm{kp}=3$ at $=12 \quad \mathrm{rc}=3.095$ /

\&POT $\mathrm{kp}=3$ type=1 shape=2 p1=83.77 p3=2.52 /

$\&$ POT $\mathrm{kp}=3$ type=3 shape=2 p1=3.8 p3=2.52 /

$\&$ POT $\mathrm{kp}=5$ at $=9$ rc=1.25 /

\&POT $\mathrm{kp}=5$ type $=1 \mathrm{p} 1=109.0$ p2 $=1.60$ p3 $=0.64$ p4 $=22.0$ p $5=1.60$ p6 $=0.64$ 
\&POT $\mathrm{kp}=6$ at $=9 \quad \mathrm{rc}=1.4 \quad /$

$\&$ POT $\mathrm{kp}=6$ type=1 p1=54.1 p2=1.846 p3=0.58 p4=4.2 p5=1.846 p6=0.58 /

$\&$ POT $\mathrm{kp}=6$ type $=3 \mathrm{p} 1=2.6 \mathrm{p} 2=1.846 \mathrm{p} 3=0.58 \quad /$

\&pot /

\&OVERLAP kn1=1 ic $1=2$ ic $2=1$ in $=1$ nn=2 l=1 $\mathrm{sn}=0.5$

ia $=1 \mathrm{j}=1.5$ ib=1 kbpot=3 be=1.586 isc=1 ipc=2 /

\&OVERLAP kn1=2 ic $1=2$ ic $2=1$ in $=1$ nn=2 l=1 $\mathrm{sn}=0.5$

ia $=1 \mathrm{j}=0.5 \quad \mathrm{ib}=2$ kbpot=3 be=1.1569 isc=1 ipc=2 /

\&overlap /

\&COUPLING icto $=1$ icfrom $=2$ kind=3 ip $1=4$ p1=5 p2=6 /

\&CFP in $=1$ ib=1 ia=1 kn=1 a=1 /

\&CFP in=1 ib=2 ia=1 kn=2 a=1 /

\&cfp /

\&coupling / 


\section{A.5 DWBA}

$9 \mathrm{Be}(7 \mathrm{Be}, 9 \mathrm{Be}) 7 \mathrm{Be}$ elastic transfer $\quad \mathrm{Ecm}=13 \mathrm{MeV}$

NAMELIST

\&FRESCO hcm $=0.050$ rmatch $=30.000$ rintp $=0.2$

$\mathrm{hnl}=0.10 \mathrm{rnl}=15.00$ centre $=-0.05 \mathrm{jtmin}=0.0 \quad \mathrm{jtmax}=60.0$

absend $=-1.000 \quad \operatorname{dry}=\mathrm{F}$ jump $(1: 6: 1)=0 \quad 0 \quad 0 \quad 0 \quad 00 \quad 0 \quad j$ bord $(1: 6)=$

$000000.0 \quad 0.0 \quad$ thmin $=1.00$ thmax $=-180.00$

thinc $=0.2$ cutl $=0.00$ cutr $=0.00$ cutc $=0.00$ ips $=0.0500$

it $0=1$ iter $=1$ pralpha $=F \quad n n u=36$ mtmin $=8$

erange $=-0.1000 \mathrm{dk}=0.0020$ chans=1 treneg=2 smats=2 xstabl=1 listcc=2

lampl=-1 pel=1 exl=1 lab=1 lin=1 lex=1 elab $(1: 4)=23.113$

$0 \quad 0$ nlab $(1: 3)=0 \quad 0 \quad 0 \quad$ fatal $=\mathrm{T}$ nosol $=\mathrm{F}$

psiren $=$ F unitmass $=1.000$ finec $=137.03599 \mathrm{~d} 0 \quad /$

\&PARTITION namep $={ }^{\prime} 7 \mathrm{Be} ' \operatorname{massp}=7.016929 \quad \mathrm{zp}=4$

namet $=$ '9Be' masst $=9.012182$ zt=4 qval $=0.0000$ pwf=T nex=1 /

\&STATES jp $=0.0$ bandp $=1$ ep $=0.0000$ cpot $=1 \quad \mathrm{jt}=0.0$

bandt $=1$ et $=0.0000$ fexch $=F \quad /$

\&PARTITION namep='9Be' $\operatorname{massp}=9.012182 \mathrm{zp}=4$

namet $=' 7 \mathrm{Be} '$ masst $=7.016929 \mathrm{zt}=4$ qval $=0.0000 \quad \mathrm{pwf}=\mathrm{T}$ nex=1 /

\&STATES jp=0.0 $\operatorname{copyp}=-1$ bandp $=1$ ep $=0.0000 \quad$ cpot $=1$

$\mathrm{jt}=0.0 \quad$ copyt $=-1$ bandt $=1$ et $=0.0000$ fexch $=\mathrm{F} \quad /$

\&partition /

$\&$ POT $\mathrm{kp}=1$ ap $=7.000$ at $=9.000$ rc $=1.201 /$

\&POT $\mathrm{kp}=1$ type=1 shape=0 p1=43.740407 p2=0.662383 p3=0.663721 /

\&POT $\mathrm{kp}=1$ type $=1 \quad$ shape $=0 \quad \mathrm{p} 4=14.028680 \quad \mathrm{p} 5=0.741799 \quad \mathrm{p} 6=1.192081 \quad /$

$\&$ POT $\mathrm{kp}=2$ itt $=\mathrm{F}$ ap $=2.000$ at $=7.000 \quad \mathrm{rc}=1.2 \quad$ /

$\&$ POT $\mathrm{kp}=2$ type=1 it $=\mathrm{F}$ p1=10. p2=1.3 p3=0.6 /

$\&$ POT $\mathrm{kp}=3$ type $=0 \quad$ it $=\mathrm{F}$ ap $=7.000$ at $=7.000 \quad \mathrm{rc}=1.2 \quad /$

$\&$ POT $\mathrm{kp}=3$ type=1 itt=F shape=0 p1=107.8 p2=0.75 p3=0.855 p4=37.9 p5=0.91 $\mathrm{p} 6=0.757 /$

\&pot / 
\&OVERLAP $\mathrm{kn} 1=1$ ic $1=2 \quad$ ic $2=1 \quad$ in $=1 \quad \mathrm{nn}=2 \quad \mathrm{sn}=0.0$

$$
\mathrm{j}=0.0 \mathrm{kbpot}=2 \text { be }=20.563 \text { isc }=1 \text { ipc }=2
$$

\&OVERLAP $\operatorname{kn} 1=2$ ic $1=1 \quad$ ic $2=2$ in $=2 \quad \mathrm{nn}=2$ sn $=0.0$

$$
\mathrm{j}=0.0 \mathrm{kbpot}=2 \text { be }=20.563 \text { isc }=1 \text { ipc }=2 \quad \text { / }
$$

\&overlap /

\&COUPLING icto=2 icfrom=1 kind=7 ip $1=-1$ ip $2=-1$ ip $3=3 /$

\&CFP in $=1$ ib=1 ia $=1 \mathrm{kn}=1 \quad \mathrm{a}=0.35 /$

\&CFP in $=2$ ib=1 ia $=1 \quad \mathrm{kn}=2 \mathrm{a}=0.35 /$

\&cfp /

\&COUPLING icto $=2$ icfrom $=1$ kind=8 ip $1=1$ ip $2=0 \quad /$

\&coupling / 